TRANSACTIONS OF THE

AMERICAN MATHEMATICAL SOCIETY

Volume 354, Number 12, Pages 4703-4749

S 0002-9947(02)03078-7

Article electronically published on July 15, 2002

\title{
INVERSE SPECTRAL THEORY OF FINITE JACOBI MATRICES
}

\author{
PETER C. GIBSON
}

\begin{abstract}
We solve the following physically motivated problem: to determine all finite Jacobi matrices $J$ and corresponding indices $i, j$ such that the Green's function

$$
\left\langle e_{j},(z I-J)^{-1} e_{i}\right\rangle
$$

is proportional to an arbitrary prescribed function $f(z)$. Our approach is via probability distributions and orthogonal polynomials.

We introduce what we call the auxiliary polynomial of a solution in order to factor the map

$$
(J, i, j) \longmapsto\left[\left\langle e_{j},(z I-J)^{-1} e_{i}\right\rangle\right]
$$

(where square brackets denote the equivalence class consisting of scalar multiples). This enables us to construct the solution set as a fibration over a connected, semi-algebraic coordinate base. The end result is a wealth of explicit constructions for Jacobi matrices. These reveal precise geometric information about the solution set, and provide the basis for new existence theorems.
\end{abstract}

\section{INTRODUCTION}

A Jacobi matrix is an irreducible, symmetric, tridiagonal matrix, either finite or infinite. We insist, in addition, that next-to-diagonal elements of a Jacobi matrix be negative. (This convention is not standard, but all our results hold equally well for the opposite sign convention.)

Spectral theory of Jacobi matrices arises in the analysis of myriad physical systems Arv93, Gib00], Gla99], [Lam97, [Tes00. In the present paper we solve a particular physically motivated problem, the physical derivation of which is explained below in Section 1.3. The problem is: to determine all finite Jacobi matrices $J$ and corresponding indices $i, j$ such that the Green's function

$$
\left\langle e_{j},(z I-J)^{-1} e_{i}\right\rangle
$$

is proportional to an arbitrary prescribed function $f(z)$. We assume that $f$ is realizable, i.e., that there exists a solution. But we place no restriction on the size of the matrix $J$. In fact, this is a family of problems, indexed by the nonnegative integer

$$
\Delta_{f}=|j-i|
$$

which turns out to be determined by $f$ and is hence an invariant of the solution set.

Received by the editors March 26, 2001.

2000 Mathematics Subject Classification. Primary 47B36; Secondary 34K29.

Supported by NSERC Postdoctoral Fellowship 231108-2000.

(C)2002 American Mathematical Society 
Proportionality can be replaced with equality in the case $\Delta_{f}=0$, which corresponds to $f$ being a Titchmarsh-Weyl $m$-function. With the accompanying restriction $i=j=1$, the case $\Delta_{f}=0$ is solved in dBG78. It is solved in GS97. without restriction, the so-called interior inverse problem, using $m$-functions as a principal tool. As far as we know, the present paper constitutes the first treatment of the cases $\Delta_{f}>0$. Using an approach based on probability distributions and orthogonal polynomials, we solve these cases collectively.

As a preliminary step, we obtain the above-mentioned result of F. Gesztesy and B. Simon in GS97, with some added geometric information. The general case $\Delta_{f}>0$ is more involved and the results are qualitatively different.

Before giving an overview of our results, we fix notation and cite some needed facts in order to restate the problem in terms of orthogonal polynomials. We will also sketch briefly the problem's physical derivation.

1.1. Problem statement. We work with the set $\mathcal{H}$ of all finite combinations over the reals of translates of the Heaviside function $H$, defined on the real line as

$$
H(x)= \begin{cases}0 & \text { if } x<0 \\ 1 & \text { if } x \geq 0\end{cases}
$$

Members of $\mathcal{H}$ are regarded as signed distribution functions on $\mathbf{R}$. We denote by $d \mathcal{H}$ the set of corresponding distributions, i.e., finite combinations of translates of the Dirac distribution $\delta$. Using the fact that the map

$$
\gamma(x)=\sum_{n=1}^{d} w_{n} H\left(x-\lambda_{n}\right) \longmapsto d \gamma(x)=\sum_{n=1}^{d} w_{n} \delta\left(x-\lambda_{n}\right)
$$

is a bijection, we will pass freely between a distribution function $\gamma$ and its corresponding distribution $d \gamma$. An important role is played by the set

$$
\mathcal{H}_{+, 1} \subseteq \mathcal{H}
$$

of nondecreasing distribution functions having maximum value 1 . These are the probability distribution functions, abbreviated p.d.f.s.

Notation. We write $d_{\gamma}$ to indicate the number of jump points of $\gamma \in \mathcal{H}$. Each nonzero $\gamma \in \mathcal{H}$ evidently has a unique representation of the form

$$
\gamma(x)=\sum_{n=1}^{d_{\gamma}} w_{n} H\left(x-\lambda_{n}\right)
$$

where $\lambda_{1}, \ldots, \lambda_{d_{\gamma}}$ are the jump points of $\gamma$ and each $w_{n} \in \mathbf{R}$.

Definition 1.1. We call the polynomial

$$
p_{\gamma}(x)=\prod_{n=1}^{d_{\gamma}}\left(\lambda_{n}-x\right)
$$

the characteristic polynomial of $\gamma$, reserving the notation $p_{\gamma}$ for this purpose. The characteristic polynomial of $\gamma=0$ is defined to be 1 .

Let $\alpha \in \mathcal{H}_{+, 1}$ be a p.d.f. Working in the space $L_{d \alpha}^{2}$, apply the Gram-Schmidt procedure to the sequence of signed moment functions

$$
1,-x, x^{2}, \ldots,(-x)^{d_{\alpha}-1} \text {. }
$$


Definition 1.2. The resulting output

$$
q_{0}^{\alpha}, q_{1}^{\alpha}, q_{2}^{\alpha}, \ldots, q_{d_{\alpha}-1}^{\alpha}
$$

is called the sequence of orthogonal polynomials generated by $\alpha$.

Note that $q_{n}^{\alpha}$ has degree $n$, and is the $(n+1)$ st term in the sequence. The notation $q_{n}^{\alpha}$ will always mean the degree $n$ orthogonal polynomial generated by $\alpha \in \mathcal{H}_{+, 1}$.

Consider the sequence of orthogonal polynomials generated by $\alpha \in \mathcal{H}_{+, 1}$, augmented by the characteristic polynomial $p_{\alpha}$ :

$$
q_{0}^{\alpha}, q_{1}^{\alpha}, \ldots, q_{d_{\alpha}-1}^{\alpha}, p_{\alpha}
$$

It is a basic fact that each polynomial in (11) has real, simple roots, and the roots of any two such polynomials interlace.

Notation. We denote the $(i, j)$-entry of a matrix $M$ by $M(i, j)$.

There is a classical connection between orthogonal polynomials and Jacobi matrices. The essential fact for us is that one can trade a finite Jacobi matrix for a member of $\mathcal{H}_{+, 1}$, and vice versa, as follows. A p.d.f. $\alpha$ in $\mathcal{H}_{+, 1}$ generates a $d_{\alpha} \times d_{\alpha}$ Jacobi matrix $J_{\alpha}$ via the formula

$$
J_{\alpha}(i, j)=\int_{-\infty}^{\infty} x q_{i-1}^{\alpha} q_{j-1}^{\alpha} d \alpha(x) .
$$

The formula generalizes to

$$
\left(J_{\alpha}^{n}\right)(i, j)=\int_{-\infty}^{\infty} x^{n} q_{i-1}^{\alpha} q_{j-1}^{\alpha} d \alpha(x)
$$

for all nonnegative integers $n$ and, if $J_{\alpha}$ is nonsingular, for all integers $n$. Conversely, every $d \times d$ Jacobi matrix $J$ is generated by a unique p.d.f. $\alpha_{J}$, its spectral distribution function, which can be constructed as follows. Orthogonally diagonalize $J$ as

$$
J=O \Lambda O^{t},
$$

where $\Lambda=\operatorname{diag}\left(\lambda_{1}, \ldots, \lambda_{d}\right)$. Then define

$$
\alpha_{J}(x)=\sum_{n=1}^{d}(O(1, n))^{2} H\left(x-\lambda_{n}\right) .
$$

The orthogonal polynomials generated by a p.d.f. $\alpha \in \mathcal{H}_{+, 1}$ are proportional to the characteristic polynomials of the leading submatrices of the corresponding Jacobi matrix $J_{\alpha}$. Letting $\left(J_{\alpha}\right)_{n}$ denote the $n \times n$ leading submatrix of $J_{\alpha}$, we have

$$
q_{n}^{\alpha}=\frac{1}{b_{1} \cdots b_{n}} \operatorname{det}\left(x I-\left(J_{\alpha}\right)_{n}\right) \quad\left(1 \leq n \leq d_{\alpha}-1\right),
$$

where $-b_{1}, \ldots,-b_{d_{\alpha}-1}$ are the next-to-diagonal elements of $J_{\alpha}$. Also, the characteristic polynomial $p_{\alpha}$ of $\alpha$, as we have defined it, is the characteristic polynomial, in the usual sense, of $J_{\alpha}$.

Fix a p.d.f. $\alpha \in \mathcal{H}_{+, 1}$ and corresponding Jacobi matrix $J=J_{\alpha}$. Write

$$
\alpha(x)=\sum_{n=1}^{d_{\alpha}} w_{n} H\left(x-\lambda_{n}\right),
$$


and orthogonally diagonalize $J$ as in (4) with $d=d_{\alpha}$. It follows from equation (3) that

$$
\sum_{n=1}^{d_{\alpha}}\left(\lambda_{n}\right)^{k} O(i, n) O(j, n)=\sum_{n=1}^{d_{\alpha}}\left(\lambda_{n}\right)^{k} q_{i-1}^{\alpha}\left(\lambda_{n}\right) q_{j-1}^{\alpha}\left(\lambda_{n}\right) w_{n}
$$

for every $k \geq 0,1 \leq i, j \leq d_{\alpha}$. Since the $\lambda_{n}$ are distinct and (7) holds for all $k \geq 0$, (7) implies that

$$
O(i, n) O(j, n)=q_{i-1}^{\alpha}\left(\lambda_{n}\right) q_{j-1}^{\alpha}\left(\lambda_{n}\right) w_{n} .
$$

The correspondence between Jacobi matrices and p.d.f.s allows us to reformulate the earlier stated problem in terms of orthogonal polynomials. Keeping $\alpha \in \mathcal{H}_{+, 1}$ and $J=J_{\alpha}$ as above, note that

$$
\left\langle e_{j},(z I-J)^{-1} e_{i}\right\rangle=\sum_{n=1}^{d_{\alpha}} \frac{O(i, n) O(j, n)}{z-\lambda_{n}} .
$$

Thus every realizable prescribed function $f$ has the form

$$
f(z)=\sum_{n=1}^{d} \frac{a_{n}}{z-\mu_{n}}
$$

for some scalars $a_{n}, \mu_{n}$, and is hence a proper rational function with simple poles. The class of all such functions sits in bijective correspondence with $d \mathcal{H}$ via the map

$$
\sum_{n=1}^{d} \frac{a_{n}}{z-\mu_{n}} \longmapsto \sum_{n=1}^{d} a_{n} \delta\left(x-\mu_{n}\right),
$$

the inverse of which is the Stieltjes transform Sim98, Appendix C]

$$
d \gamma \longmapsto \int_{-\infty}^{\infty} \frac{d \gamma(x)}{z-x}
$$

Combining (8) and (9) yields

$$
\left\langle e_{j},(z I-J)^{-1} e_{i}\right\rangle=\sum_{n=1}^{d_{\alpha}} \frac{q_{i-1}^{\alpha}\left(\lambda_{n}\right) q_{j-1}^{\alpha}\left(\lambda_{n}\right) w_{n}}{z-\lambda_{n}} .
$$

Since

$$
q_{i-1}^{\alpha} q_{j-1}^{\alpha} d \alpha=\sum_{n=1}^{d_{\alpha}} q_{i-1}^{\alpha}\left(\lambda_{n}\right) q_{j-1}^{\alpha}\left(\lambda_{n}\right) w_{n} \delta\left(x-\lambda_{n}\right),
$$

it follows that $\left\langle e_{j},(z I-J)^{-1} e_{i}\right\rangle$ is proportional to a prescribed function $f(z)$ if and only if the distribution $q_{i-1}^{\alpha} q_{j-1}^{\alpha} d \alpha$ is proportional to $d \gamma$, where $f$ and $d \gamma$ are related by

$$
f(z)=\int_{-\infty}^{\infty} \frac{d \gamma(x)}{z-x}
$$

Thus, translated in terms of orthogonal polynomials, the problem stated earlier is: to determine all p.d.f.s $\alpha \in \mathcal{H}_{+, 1}$, and corresponding indices $i, j$ such that the distribution

$$
q_{i}^{\alpha} q_{j}^{\alpha} d \alpha
$$

is proportional to an arbitrary prescribed distribution $d \gamma, \gamma \in \mathcal{H}$. We assume that $d \gamma$ is realizable, i.e., that there exists a solution. 
To view the problem in terms of maps, let

$$
\mathcal{D}=\left\{(\alpha, i, j) \mid \alpha \in \mathcal{H}_{+, 1} \text { and } 0 \leq i \leq j \leq d_{\alpha}-1\right\} .
$$

Let $d \mathcal{H} / \propto$ denote the set of equivalence classes of elements of $d \mathcal{H}$ modulo proportionality, so that a typical element has the form

$$
[d \gamma]=\{c d \gamma \mid 0 \neq c \in \mathbf{R}\},
$$

where $\gamma \in \mathcal{H}$.

\section{Definition 1.3. Let}

$$
\Phi: \mathcal{D} \longrightarrow d \mathcal{H} / \propto
$$

be defined by

$$
(\alpha, i, j) \stackrel{\Phi}{\longmapsto}\left[q_{i}^{\alpha} q_{j}^{\alpha} d \alpha\right]
$$

Main Problem. Our objective for the present paper is to describe the preimage by $\Phi$ of an arbitrary point in its range, i.e., to bijectively parametrize the set $\Phi^{-1}([d \gamma]) \subseteq \mathcal{D}$, given an arbitrary $[d \gamma] \in \operatorname{Ran}(\Phi)$.

The solution to the problem as stated at the outset of this paper, with prescribed function

$$
f(z)=\int_{-\infty}^{\infty} \frac{d \gamma(x)}{z-x}
$$

is the image of $\Phi^{-1}([d \gamma])$ by the map

$$
(\alpha, i, j) \longmapsto\left(J_{\alpha}, i+1, j+1\right) .
$$

Every realizable $f$ is proportional to the right-hand side of (10) for some $\gamma \in \mathcal{H}$. We shall not discuss numerical issues inherent in computing the map $\alpha \mapsto J_{\alpha}$ defined in (3); efficient algorithms are well known dBG78, BG87.

We use the following terminology in connection with the map $\Phi$. We refer to $\Phi^{-1}([d \gamma])$ as the solution set corresponding to known data $[d \gamma]$, and to individual elements

$$
(\alpha, i, j) \in \Phi^{-1}([d \gamma])
$$

as solutions. Since it is more convenient to work with a particular representative of $[d \gamma]$, we will generally refer to a particular distribution $d \gamma$ as the known data. Furthermore, we will assume, without loss of generality, that the known data has the form

$$
d \gamma=q_{i}^{\alpha} q_{j}^{\alpha} d \alpha
$$

for some $(\alpha, i, j) \in \mathcal{D}$.

Definition 1.4. A solution $(\alpha, i, j) \in \Phi^{-1}([d \gamma])$ is called regular if $d_{\alpha}=d_{\gamma}$, and singular if $d_{\alpha}>d_{\gamma}$.

Every solution is either regular or singular.

Definition 1.5. For $\gamma \in \mathcal{H}$, we write $\Delta_{\gamma}$ for the least value of $n$ such that the integral

$$
\int_{-\infty}^{\infty} x^{n} d \gamma
$$

is nonzero. 
If $d \gamma$ has the form (11), then it is evident that

$$
\Delta_{\gamma}=|j-i|
$$

Since the set $\mathcal{D}$ was defined such that each $(\alpha, i, j)$ satisfies $i \leq j$, the absolute value can be dropped from (12).

1.2. Overview of the paper. An essential difficulty inherent in the main problem stems from the existence of singular solutions $(\alpha, i, j) \in \Phi^{-1}([d \gamma])$. The difference $d_{\alpha}-d_{\gamma}$ measures the degree of singularity of a solution. This quantity is bounded only in the case $\Delta_{\gamma}=0$, where

$$
d_{\alpha}-d_{\gamma} \leq d_{\gamma}-1
$$

Much of the technical content of this paper can be characterized as a comprehensive analysis of this phenomenon of singularity.

In Section 2 we resolve the case $\Delta_{\gamma}=0$ and use the flip transpose to derive some needed formulas.

To solve the general case of the main problem, we exploit a particular factorization of $\Phi$, which we write as $\Phi=\pi \circ \rho$. This splits up the inverse problem of describing

$$
\Phi^{-1}([d \gamma])=\rho^{-1}\left(\pi^{-1}([d \gamma])\right)
$$

into two parts:

1. determine $\pi^{-1}([d \gamma])$;

2. determine $\rho^{-1}(X)$ for each $X \in \pi^{-1}([d \gamma])$.

Section 3 is taken up with a description of $\pi^{-1}([d \gamma])$, which we refer to as the coordinate base. It turns out to be a connected, semi-algebraic set of dimension $\Delta_{\gamma}$. Consequently, the essence of Section 3 is algebraic geometry.

Section 4 is concerned with parametrization of the fibres $\rho^{-1}(X)$. This involves a detailed analysis of how the roots of five polynomials determined by a solution are arranged relative to the roots of two polynomials determined by a a member $X \in$ $\pi^{-1}([d \gamma])$ of the coordinate base. The arguments are technical and combinatorial in flavour.

The main theorems in Section 5 assert that the construction developed in Sections 3 and 4 produces all solutions and contains no redundancy.

A great deal of information about the solution set is implicit in the construction developed in Sections 3 and 4; some of this is stated explicitly in Section 6. For instance, there exists a $\Delta_{\gamma}$-dimensional manifold of regular solutions, and, provided $\Delta_{\gamma}>0$, there exist singular solutions having $d$ jump points for every $d>d_{\gamma}$. In addition we derive an explicit formula for an infinite family of solutions, and make some remarks concerning the scope of the results.

1.3. Physical derivation. We describe the simplest mechanical setting in which our inverse problem arises: undamped coupled oscillators with linearized dynamics.

Consider a mass-spring system of the form depicted in Figure 1, consisting of $d$ particles $P_{1}, \ldots, P_{d}$, of masses $m_{1}, \ldots, m_{d}$, interconnected by springs of stiffness $k_{2}, \ldots, k_{d}$, with $P_{1}$ anchored to the left by a spring of stiffness $k_{1}$. Let $x_{n}(t), f_{n}(t)$ denote respectively the displacement of $P_{n}$ from equilibrium, and the external force applied to $P_{n}$. The equation of motion is

$$
\ddot{x}+M^{-1} K x=M^{-1} f, \quad \dot{x}(0)=x(0)=0,
$$




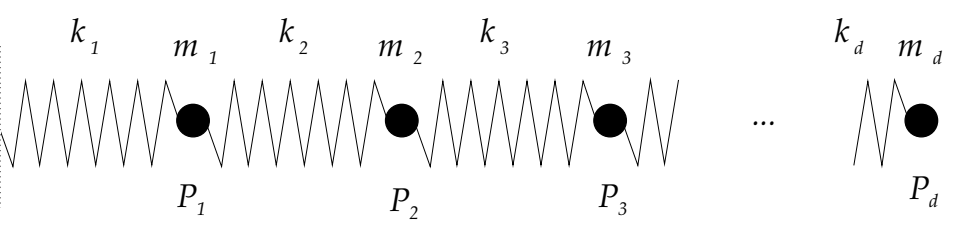

Figure 1. A mass-spring system

where

$$
\begin{aligned}
M= & \operatorname{diag}\left(m_{1}, \ldots, m_{d}\right), \\
K= & \left(\begin{array}{ccccc}
k_{1}+k_{2} & -k_{2} & 0 & \ldots & 0 \\
-k_{2} & k_{2}+k_{3} & -k_{3} & \ddots & \vdots \\
0 & -k_{3} & \ddots & \ddots & 0 \\
\vdots & \ddots & \ddots & k_{d-1}+k_{d} & -k_{d} \\
0 & \cdots & 0 & -k_{d} & k_{d}
\end{array}\right),
\end{aligned}
$$

and each $m_{n}, k_{n}>0$.

The pair $(K, M)$ completely characterizes the system. Thus we define our system space to be the set $\mathcal{S}$ of all pairs $S=(K, M)$ which, for some $d \geq 1$, conform to (14). The index $d=d_{S}$ is also a parameter of the system.

Let $G_{i j}^{S}$ denote the influence function of $P_{i}$ on $P_{j}$. That is, $G_{i j}^{S}(t)$ denotes the response $x_{j}(t)$ at $P_{j}$ to a unit impulse $\delta(t)$ applied at $P_{i}$. We are interested in the inverse problem: given $r=G_{i j}^{S}$, to what extent can one reconstruct the system $S$ ? For greater generality, we assume that the indices $i, j$ are unknown. Thus the problem is: given $r$, determine all $S \in \mathcal{S}$ and corresponding indices $i, j$ such that $G_{i j}^{S}=r$.

The coefficient matrix $M^{-1} K$ in (13) is diagonally similar to a unique Jacobi matrix,

$$
J=M^{-1 / 2} K M^{-1 / 2} .
$$

The pair $(J, M)$ determines $S$. But whereas mass and stiffness values can be chosen independently to construct a physical system $S=(K, M)$, the matrices $J, M$ are not independent.

Proposition 1.6. The pair $(M, J)$ is physical if and only if $J$ is a positive definite Jacobi matrix and $M$ is proportional to $\left(\operatorname{diag}\left(J^{-1} e_{1}\right)\right)^{2}$.

Proof. Let $u=e_{1}+\cdots+e_{d}$ denote the vector of 1's having appropriate dimension $d$. Suppose the pair $(M, J)$ comes from the system $(M, K)$ conforming to (14). Then $J$ is a Jacobi matrix and congruent to $K$, which is easily seen to be positive definite. Therefore $J$ is positive definite, by Sylvester's law of inertia. Now,

$$
\begin{aligned}
K u=M^{1 / 2} J M^{1 / 2} u & =k_{1} e_{1} \\
\Rightarrow J M^{1 / 2} u & =m_{1}^{-1 / 2} k_{1} e_{1} \\
\Rightarrow M^{1 / 2} u & =m_{1}^{-1 / 2} k_{1} J^{-1} e_{1} \\
\Rightarrow M & =m_{1}^{-1} k_{1}^{2}\left(\operatorname{diag}\left(J^{-1} e_{1}\right)\right)^{2} .
\end{aligned}
$$


Conversely, let $J$ be a positive definite Jacobi matrix and $M=c\left(\operatorname{diag}\left(J^{-1} e_{1}\right)\right)^{2}$ for some $c>0$. A positive definite Jacobi matrix is inverse positive (see Var62, p. 85]), meaning $J^{-1}>0$; so $M$ conforms to (14). Note that by hypothesis

$$
M^{1 / 2} u=c^{1 / 2} J^{-1} e_{1},
$$

from which it follows that

$$
\begin{aligned}
M^{1 / 2} J M^{1 / 2} u & =c^{1 / 2} M^{1 / 2} e_{1} \\
& =m^{1 / 2} c^{1 / 2} e_{1} .
\end{aligned}
$$

Letting $K$ denote the matrix $M^{1 / 2} J M^{1 / 2}$, we have shown that $K u$ is positively proportional to $e_{1}$, and, since $J$ is a Jacobi matrix, so is $K$. These two facts imply that $K$ has the structure (14), whence the pair $(M, J)$ is physical.

Now, solving (13), the function $G_{i j}^{S}$ expressed in terms of $J$ is

$$
G_{i j}^{S}(t)=\left(m_{i} m_{j}\right)^{-1 / 2}\left\langle e_{j}, J^{-1 / 2} \sin \left(t J^{1 / 2}\right) e_{i}\right\rangle .
$$

If $\left\langle e_{j}, J^{-1 / 2} \sin \left(t J^{1 / 2}\right) e_{i}\right\rangle$ is proportional to $r$, then automatically $J$ is positive definite, and by Proposition [1.6 there exists a unique physical choice of masses such that $G_{i j}^{S}=r$.

By this observation, the problem reduces to: given $r$, determine all Jacobi matrices $J$ such that, for some indices $i, j,\left\langle e_{j}, J^{-1 / 2} \sin \left(t J^{1 / 2}\right) e_{i}\right\rangle$ is proportional to $r$.

Observe that, for a finite Jacobi matrix $J$, the functions

$$
\left\langle e_{j}, J^{-1 / 2} \sin \left(t J^{1 / 2}\right) e_{i}\right\rangle \text { and }\left\langle e_{j},(z I-J)^{-1} e_{i}\right\rangle
$$

can be expanded using the orthogonal diagonalization of $J$ as

$$
\begin{aligned}
\left\langle e_{j}, J^{-1 / 2} \sin \left(t J^{1 / 2}\right) e_{i}\right\rangle & =\sum_{n=1}^{d} a_{n} \frac{\sin \left(t \sqrt{\lambda_{n}}\right)}{\sqrt{\lambda_{n}}}, \\
\left\langle e_{j},(z I-J)^{-1} e_{i}\right\rangle & =\sum_{n=1}^{d} \frac{a_{n}}{z-\lambda_{n}}
\end{aligned}
$$

where the same constants $a_{n}, \lambda_{n}$ occur in (16) as in (17). In terms of $\alpha=\alpha_{J}$, the expressions (16), (17) satisfy

$$
d=d_{\alpha}, \quad a_{n}=q_{i-1}^{\alpha}\left(\lambda_{n}\right) q_{j-1}^{\alpha}\left(\lambda_{n}\right) w_{n},
$$

where

$$
\alpha(x)=\sum_{n=1}^{d_{\alpha}} w_{n} H\left(x-\lambda_{n}\right) .
$$

The equivalence of the physical inverse problem just described with our main problem stated in terms of orthogonal polynomials, and with the problem stated in terms of $\left\langle e_{j},(z I-J)^{-1} e_{i}\right\rangle$ at the outset of this paper, follows directly. 


\section{Preliminaries}

In this section and beyond we make use of the following notation.

Notation. Let $A, B$ be objects that admit multiplication by a scalar. We write

$$
A \propto B
$$

to mean: there exists a real number $r>0$ such that $A=r B$. In other words, $A$ and $B$ are positively proportional.

In analogy to the Lebesgue decomposition of a measure, we use the following terminology for distribution functions $\alpha, \beta \in \mathcal{H}$. We say that $\alpha$ is absolutely continuous relative to $\beta$, and write $\alpha \ll \beta$, if every jump point of $\alpha$ is a jump point of $\beta$. We say that $\alpha$ is singular relative to $\beta$, and write $\alpha \perp \beta$, if $\alpha, \beta$ have no common jump point. Thus $\alpha$ has a unique "Lebesgue decomposition"

$$
\alpha=\alpha_{1}+\alpha_{0}
$$

relative to $\beta$, where $\alpha_{1} \ll \beta$ and $\alpha_{0} \perp \beta$.

The symbol $\mathbf{P}$ denotes the vector space of real polynomials in a single variable; $\mathbf{P}_{d}$ denotes the subspace of $\mathbf{P}$ consisting of polynomials of degree at most $d$.

2.1. The solution when $\Delta_{\gamma}=0$. In the special case $\Delta_{\gamma}=0$, a simple strategy leads directly to a parametrization of $\Phi^{-1}([d \gamma])$. Consider a solution $(\alpha, i, i) \in$ $\Phi^{-1}([d \gamma])$. Observe that

$$
\left(q_{i}^{\alpha}\right)^{-1} d \gamma \propto q_{i}^{\alpha} d \alpha
$$

Since $q_{i}^{\alpha}$ is the degree $i$ orthogonal polynomial generated by $d \alpha$, it follows that $\left(q_{i}^{\alpha}\right)^{-1} d \gamma \perp \mathbf{P}_{i-1}$. Or, writing $q=q_{i}^{\alpha}$,

$$
\forall p \in \mathbf{P}_{\operatorname{deg}(q)-1}, \quad \int_{-\infty}^{\infty} p q^{-1} d \gamma=0 .
$$

The question as to precisely that polynomials $q$ satisfy the condition (18) leads to the following definition and lemma.

Definition 2.1. Let $\gamma \in \mathcal{H}$, and write

$$
\gamma(x)=\sum_{n=1}^{d_{\gamma}} w_{n} H\left(x-\lambda_{n}\right) .
$$

Define $p^{\gamma} \in \mathbf{P}_{d_{\gamma}-1}$ to be the unique interpolator of the values $w_{n} p_{\gamma}^{\prime}\left(\lambda_{n}\right)$ at $\lambda_{n}$ $\left(1 \leq n \leq d_{\gamma}\right)$, where $p_{\gamma}^{\prime}$ denotes the derivative of $p_{\gamma}$. We call $p^{\gamma}$ the composite polynomial of $\gamma$, and reserve the notation $p^{\gamma}$ for this purpose.

Observe that $p^{\gamma}$ has the representation

$$
p^{\gamma}(x)=\sum_{n=1}^{d_{\gamma}} w_{n} p_{\gamma}(x) /\left(x-\lambda_{n}\right),
$$

which is easily verified by evaluating the latter expression at each $\lambda_{n}$.

Lemma 2.2. Let $q$ be a polynomial of degree $n$ that has no repeated roots, and let $\gamma \in \mathcal{H}$. Then

$$
\int_{-\infty}^{\infty} p q^{-1} d \gamma=0 \text { for all } p \in \mathbf{P}_{n-1}
$$

if and only if $q$ divides $p^{\gamma}$. 
Proof. It is convenient to consider the space $\mathbf{P}^{\mathbf{C}}$ of complex-valued polynomials of a real variable. (As with real-valued polynomials, we write $\mathbf{P}_{k}^{\mathbf{C}}$ to denote the subspace of $\mathbf{P}^{\mathbf{C}}$ consisting of polynomials having degree at most $k$.) Note that (19) is equivalent to

$$
\int_{-\infty}^{\infty} p q^{-1} d \gamma=0 \text { for all } p \in \mathbf{P}_{n-1}^{\mathbf{C}}
$$

where the latter integral is complex-valued. The given polynomial $q$ can be written in the form

$$
q(x)=a \prod_{k=1}^{n}\left(z_{k}-x\right)
$$

where $a \neq 0$ is a scalar, and $z_{1}, \ldots, z_{n}$ are its distinct (complex) roots. Now, observe that the $n$ distinct polynomials $q_{1}, \ldots, q_{n}$, defined by

$$
q_{k}(x)=q(x) /\left(z_{k}-x\right) \quad(1 \leq k \leq n),
$$

$\operatorname{span} \mathbf{P}_{n-1}^{\mathbf{C}}$. Thus (20) is equivalent to

$$
\int_{-\infty}^{\infty} q_{k} q^{-1} d \gamma=\int_{-\infty}^{\infty} \frac{d \gamma}{z_{k}-x}=0 \text { for each } k, \text { where } 1 \leq k \leq n .
$$

Write $\gamma(x)=\sum_{k=1}^{d_{\gamma}} w_{k} H\left(x-\lambda_{k}\right)$, and let $z$ be an arbitrary complex number. Then

$$
\begin{aligned}
\int_{-\infty}^{\infty} \frac{d \gamma}{z-x} & =\sum_{k=1}^{d_{\gamma}} \frac{w_{k}}{z-\lambda_{k}} \\
& =\frac{1}{p_{\gamma}(z)} \sum_{k=1}^{d_{\gamma}} \frac{w_{k} p_{\gamma}(z)}{z-\lambda_{k}} \\
& =p^{\gamma}(z) / p_{\gamma}(z)
\end{aligned}
$$

It follows from the definition of the composite polynomial that $p^{\gamma}\left(\lambda_{k}\right) \neq 0$ for each $k\left(1 \leq k \leq d_{\gamma}\right)$; so $p^{\gamma}$ and $p_{\gamma}$ have no common root, and

$$
\int_{-\infty}^{\infty} \frac{d \gamma}{z-x}=0 \quad \Longleftrightarrow \quad p^{\gamma}(z)=0
$$

Thus (21) is equivalent to: $p^{\gamma}\left(z_{k}\right)=0$ for each root $z_{k}$ of the polynomial $q$, completing the proof.

Note that if $[d \gamma] \in \operatorname{Ran} \Phi$ and $\Delta_{\gamma}=0$, then $\gamma$ is positive, and we can assume without loss of generality that $\gamma \in \mathcal{H}_{+, 1}$.

Theorem 2.3. Let $\gamma \in \mathcal{H}_{+, 1}$. A p.d.f. $\alpha$ satisfies $\left(q_{i}^{\alpha}\right)^{2} d \alpha=d \gamma$ for some $i$ if and only if

$$
d \alpha \propto q^{-2} d \gamma+d \alpha_{0}
$$

for some monic divisor $q$ of $p^{\gamma}$ and some nondecreasing $\alpha_{0} \in \mathcal{H}$ whose jump points occur at roots of $q$.

Proof. $(\Longrightarrow)$ Suppose that $\left(q_{i}^{\alpha}\right)^{2} d \alpha=d \gamma$. Write $\alpha=\alpha_{1}+\alpha_{0}$, where $\alpha_{1} \ll \gamma$ and $\alpha_{0} \perp \gamma$. Thus the jumps of $\alpha_{0}$ occur at zeros of $q_{i}^{\alpha}$ and the jumps of $\alpha_{1}$ do not, so that

$$
q_{i}^{\alpha} d \alpha_{1}=q_{i}^{\alpha} d \alpha
$$


and

$$
q_{i}^{\alpha} d \alpha=\left(q_{i}^{\alpha}\right)^{-1} d \gamma
$$

Now, $q_{i}^{\alpha}$ has simple zeros and, for every $p \in \mathbf{P}_{i-1}$,

$$
\int_{-\infty}^{\infty} p q_{i}^{\alpha} d \alpha=\int_{-\infty}^{\infty} p\left(q_{i}^{\alpha}\right)^{-1} d \gamma=0
$$

so Lemma 2.2 tells us that $q_{i}^{\alpha}$ divides $p^{\gamma}$. Furthermore,

$$
\begin{aligned}
d \alpha & =d \alpha_{1}+d \alpha_{0} \\
& =\left(q_{i}^{\alpha}\right)^{-2} d \gamma+d \alpha_{0} .
\end{aligned}
$$

Thus $d \alpha$ conforms to the second part of the theorem.

$(\Longleftarrow)$ Suppose conversely that: $q$ is monic of degree $i ; q$ divides $p^{\gamma} ; \alpha_{0} \in \mathcal{H}$ is nondecreasing and has jump points only at zeros of $p^{\gamma}$; and $d \alpha \propto q^{-2} d \gamma+d \alpha_{0}$ for some $\alpha \in \mathcal{H}_{+, 1}$. Note that since $\gamma \in \mathcal{H}_{+, 1}$, the zeros of $p^{\gamma}$ are real and simple, and therefore so are the zeros of $q$. Since the jumps of $\alpha_{0}$ occur at zeros of $q$,

$$
q d \alpha_{0}=0
$$

and

$$
q d \alpha \propto \pm q^{-1} d \gamma
$$

Lemma 2.2 implies

$$
\int_{-\infty}^{\infty} p q^{-1} d \gamma=0 \quad \text { for all } p \in \mathbf{P}_{i-1}
$$

and so

$$
\int_{-\infty}^{\infty} p q d \alpha=0 \quad \text { for all } p \in \mathbf{P}_{i-1}
$$

Since $q$ has degree $i$, the latter statement implies $q=r q_{i}^{\alpha}$ for some real $r \neq 0$. Thus

$$
\left(q_{i}^{\alpha}\right)^{2} d \alpha \propto d \gamma
$$

But $\left(q_{i}^{\alpha}\right)^{2} d \alpha$ is a probability distribution, and so in fact $\left(q_{i}^{\alpha}\right)^{2} d \alpha=d \gamma$.

We now fix some notation. Let $\nabla_{n}$ denote the standard $n$-dimensional simplex in $\mathbf{R}^{n+1}$. That is,

$$
\nabla_{n}=\left\{\left(t_{0}, t_{1}, \ldots, t_{n}\right) \mid t_{i} \geq 0(0 \leq i \leq n) \text { and } \sum_{i=0}^{n} t_{i}=1\right\} .
$$

By the above result, every p.d.f. is realizable as known data. The theorem says that if $\gamma \in \mathcal{H}_{+, 1}$, then $[d \gamma] \in \operatorname{Ran} \Phi$. Moreover, given $d \gamma$, one may explicitly construct $\Phi^{-1}([d \gamma])$ based on Theorem 2.3 as follows.

1. Compute the $d_{\gamma}-1$ roots of $p^{\gamma}$.

2. Choose an arbitrary subset $\sigma=\left\{r_{1}, \ldots, r_{n}\right\}$ of the roots of $p^{\gamma}$.

3. Choose an arbitrary point $\left(t_{0}, \ldots, t_{n}\right) \in \nabla_{n}$ such that $t_{0} \neq 0$.

4. Let $q_{\sigma}$ denote the simple monic polynomial having roots $\sigma$, and let $q_{\emptyset}=1$. Normalize the distribution $q_{\sigma}^{-2} d \gamma$ to obtain a p.d.f. $\alpha_{\sigma} \in \mathcal{H}_{+, 1}$.

5. Set $\alpha(\lambda)=t_{0} \alpha_{\sigma}(\lambda)+\sum_{i=1}^{n} t_{i} H\left(\lambda-r_{i}\right)$. 
Then $(\alpha, n, n) \in \Phi^{-1}([d \gamma])$, and every solution arises this way. Geometrically, the solution set $\Phi^{-1}([d \gamma])$ has the structure of a union of $2^{d_{\gamma}-1}$ simplices of dimensions ranging from 0 to $d_{\gamma}-1$, and each (except for the zero-dimensional simplex) having the face corresponding to $t_{0}=0$ deleted. See Figure 2 .

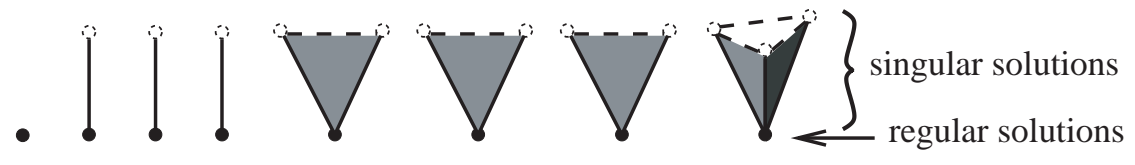

Figure 2. The solution set $\Phi^{-1}([d \gamma])$ in the case $\Delta_{\gamma}=0$

Thus each such deleted simplex includes a single vertex, corresponding to $t_{0}=1$; these $2^{d_{\gamma}-1}$ vertices are the regular solutions. More generally, $n$-dimensional solutions correspond to open, $n$-dimensional facets of the collection of deleted simplices. Indeed, counting the number of such facets of a given dimension gives the results obtained in GS97. Whereas the authors there describe the solution set as a union of manifolds diffeomorphic to open balls, we see that in fact the images by the map $J \mapsto \alpha_{J}$ of these manifolds fit together to form our simplices. The simplices themselves can be considered to fit together in a natural way, giving a rich geometric structure to the solution set. This will be treated in a forthcoming paper Gib].

Next we choose a particular p.d.f. $\gamma \in \mathcal{H}_{+, 1}$, and use Theorem 2.3 to calculate explicitly all solutions to the main problem with known data $d \gamma$. That is, we explicitly calculate the set of all p.d.f.s $\alpha$ such that for some $k,\left(q_{k}^{\alpha}\right)^{2} d \alpha \propto d \gamma$.

Set $\gamma(x)=\frac{1}{3} H(x-1)+\frac{1}{3} H(x-2)+\frac{1}{3} H(x-3)$. Then $\Delta_{\gamma}=0$ and $\gamma \in \mathcal{H}_{+, 1}$. The composite polynomial of $\gamma$ is easily calculated by hand; it works out to be

$$
p^{\gamma}(x)=-x^{2}+4 x-11 / 3 \text {. }
$$

The two roots of $p^{\gamma}$ are $r_{1}=2-\frac{1}{\sqrt{3}}$ and $r_{2}=2+\frac{1}{\sqrt{3}}$. Hence there are four monic divisors of $p^{\gamma}$ corresponding to the four subsets of $\left\{r_{1}, r_{2}\right\}: \emptyset,\left\{r_{1}\right\},\left\{r_{2}\right\},\left\{r_{1}, r_{2}\right\}$. Explicitly, we have

$$
q_{\emptyset}(x)=1, \quad q_{\left\{r_{1}\right\}}(x)=x-r_{1}, \quad q_{\left\{r_{2}\right\}}(x)=x-r_{2}, \quad q_{\left\{r_{1}, r_{2}\right\}}=-p^{\gamma} .
$$

These four divisors give rise to the four regular solutions to the main problem, by normalizing the distribution functions of the form $q^{-2} d \gamma$. Carrying out the calculation yields

$$
\begin{aligned}
\alpha_{\emptyset} & =\gamma, \\
\alpha_{\left\{r_{1}\right\}}(x) & =\frac{2+\sqrt{3}}{6} H(x-1)+\frac{1}{3} H(x-2)+\frac{2-\sqrt{3}}{6} H(x-3), \\
\alpha_{\left\{r_{2}\right\}}(x) & =\frac{2-\sqrt{3}}{6} H(x-1)+\frac{1}{3} H(x-2)+\frac{2+\sqrt{3}}{6} H(x-3), \\
\alpha_{\left\{r_{1}, r_{2}\right\}}(x) & =\frac{1}{6} H(x-1)+\frac{2}{3} H(x-2)+\frac{1}{6} H(x-3) .
\end{aligned}
$$

The singular solutions to the main problem are generated by adding to a regular solution an arbitrary nondecreasing distribution function $\alpha_{0} \in \mathcal{H}$ whose jumps are confined to the zeros of the corresponding divisor of $p^{\gamma}$, and then normalizing. Thus there is no singular solution corresponding to the regular solution $\alpha_{\emptyset}$. There is a 
one-parameter family of singular solutions corresponding to $\alpha_{\left\{r_{1}\right\}}$; this consists of all p.d.f.s of the form

$$
(1-t) \alpha_{\left\{r_{1}\right\}}(x)+t H\left(x-r_{1}\right) \quad(0<t<1) .
$$

Similarly, there is a one-parameter family of singular solutions corresponding to the regular solution $\alpha_{\left\{r_{2}\right\}}$ :

$$
(1-t) \alpha_{\left\{r_{2}\right\}}(x)+t H\left(x-r_{2}\right) \quad(0<t<1) .
$$

Since $q_{\left\{r_{1}, r_{2}\right\}}$ has two roots, there is a two-parameter family of singular solutions corresponding to $\alpha_{\left\{r_{1}, r_{2}\right\}}$ :

$$
(1-s-t) \alpha_{\left\{r_{1}, r_{2}\right\}}(x)+s H\left(x-r_{1}\right)+t H\left(x-r_{2}\right) \quad(0<s+t<1,0<s, 0<t) .
$$

This comprises a complete description of $\Phi^{-1}([d \gamma])$ with

$$
\gamma(x)=\frac{1}{3} H(x-1)+\frac{1}{3} H(x-2)+\frac{1}{3} H(x-3) .
$$

2.2. The flip transpose. Given a $d \times d$ matrix $M$, the $d \times d$ matrix $M^{f}$ defined by

$$
M^{f}(i, j)=M(d+1-j, d+1-i)
$$

is called the flip transpose of $M$. Flip transposition commutes with ordinary transposition $\left(M \mapsto M^{t}\right)$, and the composition of these two operations is a permutation, represented by the $d \times d$ matrix

$$
F=\left(\begin{array}{cccccc}
0 & \cdots & 0 & 0 & 0 & 1 \\
0 & \cdots & 0 & 0 & 1 & 0 \\
0 & \cdots & 0 & 1 & 0 & 0 \\
\vdots & & & & & \vdots \\
0 & 1 & 0 & \cdots & 0 & 0 \\
1 & 0 & \cdots & 0 & 0 & 0
\end{array}\right) .
$$

Note that $F^{2}=I$ and $M^{f t}=F M F$. The relevance of this permutation to the present considerations is expressed in the following straightforward result.

Proposition 2.4. Apart from the identity, the only permutation that leaves invariant the class of $d \times d$ Jacobi matrices is the map $J \mapsto F J F$.

Since Jacobi matrices are symmetric, the action of the permutation $F$ is simply flip transposition: $J^{f}=F J F$.

Definition 2.5. Let $\alpha \in \mathcal{H}_{+, 1}$. We define $\alpha^{f} \in \mathcal{H}_{+, 1}$ to be the unique generator of the Jacobi matrix $\left(J_{\alpha}\right)^{f}$.

Note that since $J_{\alpha}$ and $\left(J_{\alpha}\right)^{f}$ are similar, $p_{\alpha}=p_{\alpha^{f}}$.

The flip transpose allows us to establish several useful identities.

Proposition 2.6. Let $\alpha \in \mathcal{H}_{+, 1}$ have d jump points. Then

$$
q_{i}^{\alpha} q_{j}^{\alpha} d \alpha=q_{d-1-j}^{\alpha^{f}} q_{d-1-i}^{\alpha^{f}} d \alpha^{f} \quad(0 \leq i, j \leq d-1) .
$$

Proof. Let $J=J_{\alpha}$. To begin, note that for any $n \geq 0$ we have $\left(J^{f}\right)^{n}=\left(J^{n}\right)^{f}$, since $J^{f}=F J F$ and $F^{2}=I$. Now, for $1 \leq i, j \leq d$,

$$
\begin{aligned}
\left(J^{n}\right)^{f}(i, j) & =J^{n}(d+1-j, d+1-i) \quad \text { (by definition of the flip transpose) } \\
& \left.=\int_{-\infty}^{\infty} x^{n} q_{d-j}^{\alpha} q_{d-i}^{\alpha} d \alpha \quad \text { (by formula (3) }\right) .
\end{aligned}
$$


Applying the same formula (3) to $\left(J^{f}\right)^{n}$, we get

$$
\left(J^{f}\right)^{n}(i, j)=\int_{-\infty}^{\infty} x^{n} q_{i-1}^{\alpha^{f}} q_{j-1}^{\alpha^{f}} d \alpha^{f} .
$$

Thus for every $n \geq 0$ and $i, j$ in the (shifted!) range $0 \leq i, j \leq d-1$, we have

$$
\int_{-\infty}^{\infty} x^{n} q_{d-1-j}^{\alpha} q_{d-1-i}^{\alpha} d \alpha=\int_{-\infty}^{\infty} x^{n} q_{i}^{\alpha^{f}} q_{j}^{\alpha^{f}} d \alpha^{f}
$$

Since the flip transpose is an involution, we can rewrite the above statement with $\alpha$ and $\alpha^{f}$ interchanged, giving

$$
\int_{-\infty}^{\infty} x^{n} q_{i}^{\alpha} q_{j}^{\alpha} d \alpha=\int_{-\infty}^{\infty} x^{n} q_{d-1-j}^{\alpha^{f}} q_{d-1-i}^{\alpha^{f}} d \alpha^{f} \quad(n \geq 0 \quad \text { and } \quad 0 \leq i, j \leq d-1) .
$$

The proposition follows.

In the special case $i=j=0$, we get

$$
\left(q_{0}^{\alpha}\right)^{2} d \alpha=d \alpha=\left(q_{d-1}^{\alpha^{f}}\right)^{2} d \alpha^{f} .
$$

Rewriting this as

$$
d \alpha^{f}=\left(q_{d-1}^{\alpha^{f}}\right)^{-2} d \alpha+0,
$$

Theorem 2.3 applies, with $\alpha^{f}, \alpha$ in the roles of $\alpha, \gamma$ respectively, and $\alpha_{0}=0$. The theorem says that $q_{d-1}^{\alpha^{f}}$ divides $p^{\alpha}$. But $p^{\alpha}$ and $q_{d-1}^{\alpha^{f}}$ have the same degree; therefore,

$$
q_{d-1}^{\alpha^{f}} \propto \pm p^{\alpha} \text {. }
$$

An examination of the definition of $p^{\alpha}$ easily reveals that, in fact,

$$
q_{d-1}^{\alpha^{f}} \propto-p^{\alpha} .
$$

Notation. Given a polynomial $p$ of degree $d$ and whose leading coefficient is $c$, let $\bar{p}$ denote the polynomial $\frac{(-1)^{d}}{c} p$. With this notation, the leading coefficients of $p^{\gamma}$ and $\overline{p^{\gamma}}$ have opposite sign.

We are now in a position to prove some needed technical results.

Let $\alpha \in \mathcal{H}_{+, 1}$ have $d$ jump points and write $\alpha(x)=\sum_{n=1}^{d} w_{n} H\left(x-\lambda_{n}\right)$. Let $-b_{1}, \ldots,-b_{d-1}$ denote the next-to-diagonal entries of $J_{\alpha}$.

Proposition 2.7. For every $m(1 \leq m \leq d-1)$, for every jump point $\lambda_{n}$ of $\alpha$,

$$
w_{n} p_{\alpha}^{\prime}\left(\lambda_{n}\right) \overline{q_{m}^{\alpha}}\left(\lambda_{n}\right)=-\left(b_{1} \cdots b_{m}\right)^{2} \overline{q_{d-1-m}^{\alpha f}}\left(\lambda_{n}\right) .
$$

Proof. We know by (6) that $q_{m}^{\alpha}=\overline{q_{m}^{\alpha}} /\left(b_{1} \cdots b_{m}\right)$, and by the same token, $q_{d-1-m}^{\alpha^{f}}=$ $\overline{q_{d-1-m}^{\alpha^{f}}} /\left(b_{m+1} \cdots b_{d-1}\right)$. Note that, since $\alpha \in \mathcal{H}_{+, 1}, p^{\alpha}=-\overline{p^{\alpha}}$. Therefore, by (23), $p^{\alpha}=-\overline{q_{d-1}^{\alpha^{f}}}$.

Proposition (2.6) with $i=m$ and $j=0$ yields

$$
q_{m}^{\alpha} d \alpha=q_{d-1-m}^{\alpha^{f}} q_{d-1}^{\alpha^{f}} d \alpha^{f} .
$$


By (22) we can replace $d \alpha$ by $\left(q_{d-1}^{\alpha^{f}}\right)^{2} d \alpha^{f}$, giving

$$
\begin{aligned}
q_{m}^{\alpha}\left(q_{d-1}^{\alpha^{f}}\right)^{2} d \alpha^{f} & =q_{d-1-m}^{\alpha^{f}} q_{d-1}^{\alpha^{f}} d \alpha^{f} \\
\Longrightarrow q_{m}^{\alpha} q_{d-1}^{\alpha^{f}} & =q_{d-1-m}^{\alpha^{f}} \quad\left(\bmod p_{\alpha^{f}}, \text { or equivalently, } \bmod p_{\alpha}\right) \\
\Longrightarrow-\overline{q_{m}^{\alpha}} p^{\alpha} & =\left(b_{1} \cdots b_{m}\right)^{2} \overline{q_{d-1-m}^{\alpha^{f}}} \quad\left(\bmod p_{\alpha}\right)
\end{aligned}
$$

The proposition then follows from the fact that $p^{\alpha}\left(\lambda_{n}\right)=w_{n} p_{\alpha}^{\prime}\left(\lambda_{n}\right)$.

Note that the coefficient $\left(b_{1} \cdots b_{m}\right)^{2}$ is independent of $n$.

Corollary 2.8. Provided $q_{m}^{\alpha}\left(\lambda_{n}\right) \neq 0$,

$$
w_{n}=\frac{-\left(b_{1} \cdots b_{m}\right)^{2} \overline{q_{d-1-m}^{\alpha^{f}}}\left(\lambda_{n}\right)}{p_{\alpha}^{\prime}\left(\lambda_{n}\right) \overline{q_{m}^{\alpha}}\left(\lambda_{n}\right)} .
$$

Corollary 2.8 provided an essential insight leading ultimately to the construction presented in this paper. The idea is roughly as follows. By the corollary, unless $q_{i}^{\alpha}\left(\lambda_{n}\right)=q_{j}^{\alpha}\left(\lambda_{n}\right)=0$, the weight $w_{n}$ associated with $\lambda_{n}$ in $\alpha$ is given by one of the two rational functions

$$
r_{i}=\frac{-\left(b_{1} \cdots b_{i}\right)^{2} \overline{q_{d-1-i}^{\alpha^{f}}}}{p_{\alpha}^{\prime} \overline{q_{i}^{\alpha}}}, \quad r_{j}=\frac{-\left(b_{1} \cdots b_{j}\right)^{2} \overline{q_{d-1-j}^{\alpha^{f}}}}{p_{\alpha}^{\prime} \overline{q_{j}^{\alpha}}}
$$

evaluated at $\lambda_{n}$. We will show much later that, in fact,

$$
w_{n}=\left.\max \left\{r_{i}, r_{j}\right\}\right|_{\lambda_{n}} .
$$

On the other hand, at points $\lambda_{n}$ where $q_{i}^{\alpha}\left(\lambda_{n}\right)=q_{j}^{\alpha}\left(\lambda_{n}\right)=0$, the weight $w_{n}$ has no influence on the distribution $d \gamma=q_{i}^{\alpha} q_{j}^{\alpha} d \alpha$. That is, we could replace $w_{n}$ with an arbitrary $w_{n}^{\prime} \geq 0$, and the same distribution $d \gamma$ would result - this phenomenon occurred already in the case $\Delta_{\gamma}=0$. Thus, essentially, the way we will construct a solution from $d \gamma$ is through the intermediate step of constructing rational functions playing the role of $r_{i}$ and $r_{j}$.

An additional technical point is relevant. Note that $r_{i}$ and $r_{j}$ agree at jump points $\lambda_{n}$ where $q_{i}^{\alpha}\left(\lambda_{n}\right) q_{j}^{\alpha}\left(\lambda_{n}\right) \neq 0$. This means that the function $\max \left\{r_{i}, r_{j}\right\}$ is determined up to a scalar multiple by the polynomials

$$
p_{\alpha}^{\prime}, \overline{q_{d-1-i}^{\alpha^{f}}}, \overline{q_{i}^{\alpha}}, \overline{q_{d-1-j}^{\alpha^{f}}}, \overline{q_{j}^{\alpha}}
$$

it is not necessary to know the constants $\left(b_{1} \cdots b_{i}\right)^{2},\left(b_{1} \cdots b_{j}\right)^{2}$. Moreover, the polynomials (24) are determined by the roots of

$$
p_{\alpha}, q_{i}^{\alpha}, q_{j}^{\alpha}, q_{d-1-i}^{\alpha^{f}}, q_{d-1-j}^{\alpha^{f}} .
$$

\section{Factorization of THE MAP $\Phi$}

3.1. Some technical results. In Theorem 3.2 of this subsection we derive two formulas which express the composite polynomial $p^{\gamma}$ in terms of an arbitrary solution $(\alpha, i, j) \in \Phi^{-1}([d \gamma])$. The first formula is exact; the second is valid modulo the characteristic polynomial $p_{\gamma}$ and motivates our definition of the auxiliary polynomial of a solution.

Lemma 3.1. Let $\alpha \in \mathcal{H}_{+, 1}$ have d jump points $\lambda_{1}<\lambda_{2}<\ldots<\lambda_{d}$. Fix $0 \leq i<d$. The locations of the roots of $q_{i}^{\alpha}$ and $q_{d-1-i}^{\alpha^{f}}$ are related as follows.

(i) For each $n, q_{i}^{\alpha}\left(\lambda_{n}\right)=0$ if and only if $q_{d-1-i}^{\alpha^{f}}\left(\lambda_{n}\right)=0$. 
(ii) For each $n \leq d-1$, the polynomial $q_{i}^{\alpha}$ has a root in the open interval $\left(\lambda_{n}, \lambda_{n+1}\right)$ if and only if $q_{d-1-i}^{\alpha^{f}}$ has no root in the closed interval $\left[\lambda_{n}, \lambda_{n+1}\right]$.

Proof. Interlacing of orthogonal polynomials implies that the sequence

$$
q_{d-1}^{\alpha^{f}}\left(\lambda_{1}\right), q_{d-1}^{\alpha^{f}}\left(\lambda_{2}\right), \ldots, q_{d-1}^{\alpha^{f}}\left(\lambda_{d}\right)
$$

has $d-1$ sign changes, the maximum possible. In particular, $q_{d-1}^{\alpha^{f}}\left(\lambda_{n}\right) \neq 0$ for each $n$. Now, consider Proposition 2.6 with $j=0: q_{i}^{\alpha} d \alpha=q_{d-1}^{\alpha^{f}} q_{d-1-i}^{\alpha^{f}} d \alpha^{f}$. Thus $q_{i}^{\alpha}\left(\lambda_{n}\right)=0$ if and only if $q_{d-1}^{\alpha^{f}}\left(\lambda_{n}\right) q_{d-1-i}^{\alpha^{f}}\left(\lambda_{n}\right)=0$, which happens if and only if $q_{d-1-i}^{\alpha^{f}}\left(\lambda_{n}\right)=0$, proving part (i) of the lemma. Moreover, for each $n, q_{i}^{\alpha}\left(\lambda_{n}\right)$ and $q_{d-1}^{\alpha^{f}}\left(\lambda_{n}\right) q_{d-1-i}^{\alpha^{f}}\left(\lambda_{n}\right)$ have the same sign. Note in addition that, since the roots of $q_{i}^{\alpha}$, and also the roots of $q_{d-1-i}^{\alpha^{f}}$, interlace with $\lambda_{1}, \ldots, \lambda_{d}$ (which are the roots of $p_{\alpha}=p_{\alpha^{f}}$ ), each of $q_{i}^{\alpha}$ and $q_{d-1-i}^{\alpha^{f}}$ has at most one root (necessarily simple) in an interval of the form $\left[\lambda_{n}, \lambda_{n+1}\right]$. Thus the sequence $q_{i}^{\alpha}\left(\lambda_{1}\right), \ldots, q_{i}^{\alpha}\left(\lambda_{d}\right)$ has a sign change from $\lambda_{n}$ to $\lambda_{n+1}$ if and only if the sequence $q_{d-1-i}^{\alpha^{f}}\left(\lambda_{1}\right), \ldots, q_{d-1-i}^{\alpha^{f}}\left(\lambda_{d}\right)$ has the same sign at $\lambda_{n}$ as at $\lambda_{n+1}$. Part (ii) of the lemma follows.

For the statement of the next result we invoke the convention that the characteristic polynomial of $\alpha_{0}=0$ is $p_{\alpha_{0}}=1$.

Theorem 3.2. Let $\alpha \in \mathcal{H}_{+, 1}$ have $d$ jump points, and let $-b_{1}, \ldots,-b_{d-1}$ be the next-to-diagonal entries of $J_{\alpha}$. Fix indices $i, j$ such that $0 \leq i \leq j<d$, and define $\gamma \in \mathcal{H}$ by $d \gamma=q_{i}^{\alpha} q_{j}^{\alpha} d \alpha$. Let $\alpha=\alpha_{1}+\alpha_{0}$ be the Lebesgue decomposition of $\alpha$ with respect to $\gamma$. Then $p_{\alpha_{0}}$ divides both $q_{i}^{\alpha} q_{d-1-j}^{\alpha^{f}}$ and $q_{j}^{\alpha} q_{d-1-i}^{\alpha^{f}}$, and

$$
\begin{aligned}
& \text { (a) } p^{\gamma}=-\left(b_{1} \cdots b_{d-1}\right) q_{i}^{\alpha} q_{d-1-j}^{\alpha^{f}} / p_{\alpha_{0}} ; \\
& \text { (b) } p^{\gamma}=-\left(b_{1} \cdots b_{d-1}\right) q_{j}^{\alpha} q_{d-1-i}^{\alpha^{f}} / p_{\alpha_{0}}\left(\bmod p_{\gamma}\right) .
\end{aligned}
$$

Proof. In terms of characteristic polynomials, we have $p_{\gamma}=p_{\alpha_{1}}$ and $p_{\alpha}=p_{\alpha_{1}} p_{\alpha_{0}}=$ $p_{\gamma} p_{\alpha_{0}}$. Note that $d=d_{\alpha}$. In terms of the numbers of jump points (equivalently, degrees of the characteristic polynomials), we have $d_{\gamma}=d_{\alpha_{1}}$ and $d_{\alpha}=d_{\alpha_{1}}+d_{\alpha_{0}}=$ $d_{\gamma}+d_{\alpha_{0}}$.

Now, write

$$
\alpha(x)=\sum_{n=1}^{d_{\alpha}} w_{n} H\left(x-\lambda_{n}\right) .
$$

Then, by definition,

$$
\gamma(x)=\sum_{n=1}^{d_{\alpha}} q_{i}^{\alpha}\left(\lambda_{n}\right) q_{j}^{\alpha}\left(\lambda_{n}\right) w_{n} H\left(x-\lambda_{n}\right) .
$$

The jump points of $\alpha_{0}$ are precisely the jump points $\lambda_{n}$ of $\alpha$ at which $q_{i}^{\alpha}\left(\lambda_{n}\right) q_{j}^{\alpha}\left(\lambda_{n}\right)$ $=0$. Thus $p_{\alpha_{0}}$ divides $q_{i}^{\alpha} q_{j}^{\alpha}$. By part (i) of Lemma 3.1. $p_{\alpha_{0}}$ therefore also divides each of $q_{i}^{\alpha} q_{d-1-j}^{\alpha^{f}}$ and $q_{j}^{\alpha} q_{d-1-i}^{\alpha^{f}}$.

Observe that $p_{\gamma}^{\prime} / p_{\alpha}^{\prime}=p_{\gamma}^{\prime} /\left(p_{\gamma}^{\prime} p_{\alpha_{0}}+p_{\gamma} p_{\alpha_{0}}^{\prime}\right)$, so that at each jump point $\lambda_{n}$ of $\gamma$ (equivalently, root of $\left.p_{\gamma}\right), p_{\gamma}^{\prime}\left(\lambda_{n}\right) / p_{\alpha}^{\prime}\left(\lambda_{n}\right)=1 / p_{\alpha_{0}}\left(\lambda_{n}\right)$. Consider now the value $p^{\gamma}\left(\lambda_{n}\right)$ of the composite polynomial $p^{\gamma}$ at each jump point $\lambda_{n}$ of $\gamma$. At each such 
point $\lambda_{n}$

$$
\begin{aligned}
p^{\gamma}\left(\lambda_{n}\right) & =q_{i}^{\alpha}\left(\lambda_{n}\right) q_{j}^{\alpha}\left(\lambda_{n}\right) w_{n} p_{\gamma}^{\prime}\left(\lambda_{n}\right) \\
& =q_{i}^{\alpha}\left(\lambda_{n}\right) q_{j}^{\alpha}\left(\lambda_{n}\right) w_{n} p_{\alpha}^{\prime}\left(\lambda_{n}\right) p_{\gamma}^{\prime}\left(\lambda_{n}\right) / p_{\alpha}^{\prime}\left(\lambda_{n}\right) \\
& =q_{i}^{\alpha}\left(\lambda_{n}\right) q_{j}^{\alpha}\left(\lambda_{n}\right) w_{n} p_{\alpha}^{\prime}\left(\lambda_{n}\right) / p_{\alpha_{0}}\left(\lambda_{n}\right) .
\end{aligned}
$$

By Proposition 2.7 and (6) , we can replace $q_{j}^{\alpha}\left(\lambda_{n}\right) w_{n} p_{\alpha}^{\prime}\left(\lambda_{n}\right)$ with

$$
-\left(b_{1} \cdots b_{d-1}\right) \overline{q_{d-1-j}^{\alpha^{f}}}\left(\lambda_{n}\right)
$$

to yield

$$
\begin{aligned}
p^{\gamma}\left(\lambda_{n}\right) & =-\left(b_{1} \cdots b_{j}\right) q_{i}^{\alpha}\left(\lambda_{n}\right) \overline{q_{d-1-j}^{\alpha^{f}}}\left(\lambda_{n}\right) / p_{\alpha_{0}}\left(\lambda_{n}\right) \\
& =-\left(b_{1} \cdots b_{d-1}\right) q_{i}^{\alpha}\left(\lambda_{n}\right) q_{d-1-j}^{\alpha^{f}}\left(\lambda_{n}\right) / p_{\alpha_{0}}\left(\lambda_{n}\right),
\end{aligned}
$$

again using (6). Thus,

$$
p^{\gamma}=-\left(b_{1} \cdots b_{d-1}\right) q_{i}^{\alpha} q_{d-1-j}^{\alpha^{f}} / p_{\alpha_{0}} \quad\left(\bmod p_{\alpha}\right),
$$

and the same is true $\bmod p_{\gamma}\left(\right.$ since $p_{\gamma}$ divides $\left.p_{\alpha}\right)$.

Similarly, interchanging the roles of $i$ and $j$,

$$
p^{\gamma}=-\left(b_{1} \cdots b_{d-1}\right) q_{j}^{\alpha} q_{d-1-i}^{\alpha^{f}} / p_{\alpha_{0}} \quad\left(\bmod p_{\gamma}\right) .
$$

Note that, by assumption, $i \leq j$. Therefore, the degree of the right-hand side of (25) is

$$
d_{\alpha}-1-\Delta_{\gamma}-d_{\alpha_{0}}=d_{\gamma}-1-\Delta_{\gamma},
$$

and $p^{\gamma} \in \mathbf{P}_{d_{\gamma}-1}$ is the unique interpolator of the values taken by the right-hand side at the jump points of $\gamma$. It follows that (25) holds without the restriction mod $p_{\gamma}$. On the other hand, the right-hand side of (26) has degree

$$
d_{\alpha}-1+\Delta_{\gamma}-d_{\alpha_{0}}=d_{\gamma}-1+\Delta_{\gamma}
$$

so the proportionality is, in general, only valid $\bmod p_{\gamma}$.

Part (a) of Theorem 3.2 is related to a well-known formula, stated in GS97. Proposition 2.2] and [Tes00, Equation 1.99]. As an aside, we point out the connection. In our notation, the related formula is as follows. For $(\alpha, i, j) \in \mathcal{D}$,

$$
\left\langle e_{j+1},\left(z I-J_{\alpha}\right)^{-1} e_{i+1}\right\rangle=q_{i}^{\alpha}(z) q_{d-1-j}^{\alpha^{f}}(z) / p_{\alpha}(z) .
$$

Since

$$
\left\langle e_{j+1},\left(z I-J_{\alpha}\right)^{-1} e_{i+1}\right\rangle=\int_{-\infty}^{\infty} \frac{d \gamma(x)}{z-x},
$$

where $d \gamma=q_{i}^{\alpha} q_{j}^{\alpha} d \alpha$, the proof of Lemma 2.2 shows that the right-hand side of [27] reduces, after cancellation, to $p^{\gamma} / p_{\gamma}$. It turns out that the difference $d_{\alpha}-d_{\gamma}$ can be large; so the cancellation may involve many terms. In [GS97] and [Tes00], the denominator of the right-hand side of (27) is expressed as a Wronskian, and the fact that it is the characteristic polynomial of $\alpha$ is not immediately obvious.

To close out the present section, we return briefly to the relationship between $\alpha$ and $\alpha^{f}$. 
Proposition 3.3. For $\alpha \in \mathcal{H}_{+, 1}$,

$$
p^{\alpha} p^{\alpha^{f}}=\left(b_{1} \cdots b_{d-1}\right)^{2}\left(\bmod p_{\alpha}\right),
$$

where $-b_{1}, \ldots,-b_{d-1}$ are the next-to-diagonal entries of $J_{\alpha}$.

Proof. By (22) we have $d \alpha=\left(q_{d-1}^{\alpha^{f}}\right)^{2} d \alpha^{f}$; applying the same identity to $\alpha^{f}$ yields

$$
d \alpha=\left(q_{d-1}^{\alpha^{f}}\right)^{2}\left(q_{d-1}^{\alpha}\right)^{2} d \alpha,
$$

from which it follows that

$$
q_{d-1}^{\alpha^{f}} q_{d-1}^{\alpha}=1 \quad\left(\bmod p_{\alpha}\right) .
$$

The result then follows from equation (6).

Note that, given $p^{\alpha}$, the property $\overline{p^{\alpha^{f}}}=p^{\alpha^{f}}$, together with the fact that $p^{\alpha} p^{\alpha^{f}}=$ const. $\left(\bmod p_{\alpha}\right)$, completely determines $p^{\alpha^{f}}$.

\subsection{The auxiliary polynomial of a solution.}

Definition 3.4. Let $(\alpha, i, j) \in \mathcal{D}$, and let $\alpha=\alpha_{1}+\alpha_{0}$ be the Lebesgue decomposition of $\alpha$ with respect to $\gamma$, where $d \gamma=q_{i}^{\alpha} q_{j}^{\alpha} d \alpha$. Based on part (b) of Theorem 3.2 define the auxiliary polynomial of $(\alpha, i, j)$ to be

$$
q^{(\alpha, i, j)}=-\left(b_{1} \cdots b_{d-1}\right) q_{j}^{\alpha} q_{d-1-i}^{\alpha^{f}} / p_{\alpha_{0}},
$$

where $-b_{1}, \ldots,-b_{d-1}$ are the next-to-diagonal entries of $J_{\alpha}$. We reserve the notation $q^{(\alpha, i, j)}$ for this purpose.

The auxiliary polynomial serves to factor the map

$$
\Phi: \mathcal{D} \longrightarrow d \mathcal{H} / \propto
$$

into two components $\Phi=\pi \circ \rho$ as depicted in Figure 3 .

The map

$$
\rho: \mathcal{D} \longrightarrow(d \mathcal{H} / \propto) \times \mathbf{P}
$$

is defined by

$$
(\alpha, i, j) \stackrel{\rho}{\longmapsto}\left(\Phi(\alpha, i, j), q^{(\alpha, i, j)}\right) .
$$

Also, $\pi:(d \mathcal{H} / \propto) \times \mathbf{P} \longrightarrow d \mathcal{H} / \propto$ is the projection onto the first coordinate,

$$
([d \gamma], q) \stackrel{\pi}{\longmapsto}[d \gamma] .
$$

Factoring $\Phi$ breaks up the original inverse problem into two parts: first, determine $\pi^{-1}([d \gamma])$; then, for each point $X=([d \gamma], q) \in \pi^{-1}([d \gamma])$, determine $\rho^{-1}(X)$. Thinking of $\rho^{-1}(X)$ as a fibre over $X$, we will ultimately express the solution set $\Phi^{-1}([d \gamma])$ as a fibration over $\pi^{-1}([d \gamma])$ by means of the construction in Section 4.

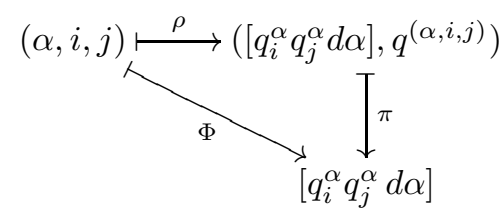

FIGURE 3. Factorization of $\Phi$ 
At first glance the given factorization may seem frivolous, since it simply adds some auxiliary information and then discards it. But, in fact, it turns out to be especially useful. It separates the algebraic from the combinatorial aspects of the original problem, and each of the two factors taken separately gives rise to a tractable inverse problem.

A crucial point is that the degree of $q^{(\alpha, i, j)}$ is $d_{\gamma}-1+\Delta_{\gamma}$, which controls the dimension of the preimage by $\pi$ of a point in $\operatorname{Ran} \Phi$, that is,

$$
\pi^{-1}([d \gamma]) \subseteq\{[d \gamma]\} \times \mathbf{P}_{d_{\gamma}-1+\Delta_{\gamma}} .
$$

The factor $\pi$ thus avoids the awkwardness of having no a priori bound on the dimension of the relevant inverse images; difficulties with dimension are concentrated in the factor $\rho$. We will show that $\pi^{-1}([d \gamma])$ is in fact a connected, semi-algebraic set of dimension $\Delta_{\gamma}$. In this sense the inverse problem associated with the factor $\pi$ is algebraic in character.

On the other hand, construction of the fibres $\rho^{-1}(X)$, taken up in Section 4, is essentially a combinatorial problem, involving a detailed analysis of realizable patterns of roots.

Part (b) of Theorem 3.2 shows that $q^{(\alpha, i, j)}=p^{\gamma}\left(\bmod p_{\gamma}\right)$, and we noted already that $\operatorname{deg}\left(q^{(\alpha, i, j)}\right)=d_{\gamma}-1+\Delta_{\gamma}$. The definition of $q^{(\alpha, i, j)}$ in terms of orthogonal polynomials implies that the roots of $q^{(\alpha, i, j)}$ are real with multiplicity at most two. We need just one further property of the auxiliary polynomial $q^{(\alpha, i, j)}$ before we can describe $\pi^{-1}([d \gamma])$ in terms of $[d \gamma]$. We fix notation as follows.

Notation. Let $(\alpha, i, j) \in \mathcal{D}$ and $d \gamma=q_{i}^{\alpha} q_{j}^{\alpha} d \alpha$. To distinguish the jump points of $\alpha$ from those of $\gamma$, we indicate jump points of $\gamma$ by a prime. Thus let $\lambda_{1}<\ldots<\lambda_{d_{\alpha}}$ denote the jump points of $\alpha$, and let $\lambda_{1}^{\prime}<\ldots<\lambda_{d_{\gamma}}^{\prime}$ denote those of $\gamma$, so that $\Lambda^{\prime}=\left\{\lambda_{1}^{\prime}, \ldots, \lambda_{d_{\gamma}}^{\prime}\right\}$ is a subset of $\Lambda=\left\{\lambda_{1}, \ldots, \lambda_{d_{\alpha}}\right\}$.

Definition 3.5. We refer to an open or closed interval determined by consecutive jump points of $\gamma$ as a $\gamma$-interval.

Proposition 3.6. For every solution $(\alpha, i, j) \in \Phi^{-1}([d \gamma])$, the polynomial $p=$ $p^{\gamma} q^{(\alpha, i, j)}$ has precisely two roots in each $\gamma$-interval $I_{n}=\left(\lambda_{n}^{\prime}, \lambda_{n+1}^{\prime}\right)$.

Proof. We claim that $p$ has at least one root in each $\gamma$-interval. By part (ii) of Lemma $3.1 p\left(p_{\alpha_{0}}\right)^{2}=q_{i}^{\alpha} q_{d-1-j}^{\alpha^{f}} q_{j}^{\alpha} q_{d-1-i}^{\alpha^{f}}$ has at least two roots in $I_{n}$. Any of these that is not a root of $p_{\alpha_{0}}$, i.e., not a jump point of $\alpha$, is a root of $p$. Suppose on the other hand that every root of $p\left(p_{\alpha_{0}}\right)^{2}$ in $I_{n}$ is a root of $p_{\alpha_{0}}$. Then the rightmost such root, $r$, is a jump point of $\alpha$. Part (ii) of Lemma 3.1 applied to the interval $\left[r, \lambda_{n+1}^{\prime}\right]$ forces $r$ to be a root of both $q_{i}^{\alpha} q_{d-1-i}^{\alpha^{f}}$ and $q_{j}^{\alpha} q_{d-1-f}^{\alpha^{f}}$; part (i) then forces $r$ to be a double root of both. But then $r$ is necessarily a (double) root of $p$, proving the claim.

Note that $p$ must, in fact, have an even number of roots in each $\gamma$-interval $I_{n}$, since $p=\left(p^{\gamma}\right)^{2}\left(\bmod p_{\gamma}\right)$ does not change sign from $\lambda_{n}^{\prime}$ to $\lambda_{n+1}^{\prime}$. So $p$ has at least two roots in each $\gamma$-interval, and not more than two since, by the proof of Theorem 3.2 , $\operatorname{deg}(p)=2\left(d_{\gamma}-1\right)$, twice the number of $\gamma$-intervals.

The lemma shows that each $\gamma$-interval contains either 0,1 or 2 roots of $p^{\gamma}$, and either 0,1 or 2 roots of $q^{(\alpha, i, j)}$, the sum of these two values being 2. Given $\gamma$ (or $d \gamma$ ), we can compute the roots of $p^{\gamma}$ and thereby determine precisely how many roots of $q^{(\alpha, i, j)}$ lie in each $\gamma$-interval. 


\subsection{The coordinate base $\mathcal{C}_{\gamma}$.}

Definition 3.7. Let $d \gamma=q_{i}^{\alpha} q_{j}^{\alpha} d \alpha$ for some $(\alpha, i, j) \in \mathcal{D}$. Define $\mathcal{C}_{\gamma} \subseteq \mathbf{P}_{d_{\gamma}-1+\Delta_{\gamma}}$ to be the set of polynomials $q$ such that

(i) $p^{\gamma} q$ has precisely 2 roots in each $\gamma$-interval, and no other roots;

(ii) $q=p^{\gamma}\left(\bmod p_{\gamma}\right)$.

We call $\mathcal{C}_{\gamma}$ the coordinate base of $d \gamma$.

Note that we have defined $\mathcal{C}_{\gamma}$ in terms of a specific representative of $[d \gamma]$. We emphasize once again that this results in no loss of generality. Choosing another representative $\widetilde{\gamma} \in[d \gamma]$ would result in $p^{\widetilde{\gamma}} \propto p^{\gamma}$ and hence rescale each $q \in \mathcal{C}_{\gamma}$, but without moving its roots. This has no effect on our main construction, which uses only the roots of $p^{\gamma}$ and each $q \in \mathcal{C}_{\gamma}$, and not their values. We have already proved the following.

Proposition 3.8. For $d \gamma=q_{i}^{\alpha} q_{j}^{\alpha} d \alpha,(\alpha, i, j) \in \mathcal{D}$,

$$
\pi^{-1}([d \gamma]) \subseteq\{[d \gamma]\} \times \mathcal{C}_{\gamma} .
$$

In fact, $\pi^{-1}([d \gamma])=\{[d \gamma]\} \times \mathcal{C}_{\gamma}$, but the proof (Theorem [5.10) has to await our construction of the fibres $\rho^{-1}([d \gamma], q), q \in \mathcal{C}_{\gamma}$.

The coordinate base $\mathcal{C}_{\gamma}$ provides the foundation for our construction of the solution set $\Phi^{-1}([d \gamma])$. We are interested in describing the geometry of $\Phi^{-1}([d \gamma])$, and this requires in turn that we analyse $\mathcal{C}_{\gamma}$ from a geometric perspective. To that end, the remainder of the present section constitutes something of a detour from our development of the main construction, the thread of which we take up again in Section 4.

The most basic fact is that $\mathcal{C}_{\gamma}$ is a semi-algebraic set, and hence a finite union of real analytic manifolds. The map that carries the roots of a polynomial to its coefficients plays a central role here. In this connection we fix notation as follows.

Notation. For $r=\left(r_{1}, \ldots, r_{n}\right) \in \mathbf{R}^{n}$, set

$$
(\mathbf{p}(r))(x)=\prod_{i=1}^{n}\left(r_{i}-x\right) .
$$

The notation is chosen so that $\overline{\mathbf{p}(r)}=\mathbf{p}(r)$. Warning: We use $\prod$ to denote a product of binomials, as above, and also the Cartesian product of sets, below. It should be clear from the context which use is intended. We indicate the $n$-fold Cartesian product of an interval $I$ with itself by $I^{n}$. For $d \gamma=q_{i}^{\alpha} q_{j}^{\alpha} d \alpha,(\alpha, i, j) \in \mathcal{D}$, set

$$
\mathcal{R}_{\gamma}=\prod_{n=1}^{d_{\gamma}-1}\left(\lambda_{n}^{\prime}, \lambda_{n+1}^{\prime}\right)^{i_{n}}
$$

where

$$
i_{n}= \begin{cases}2 & \text { if } p^{\gamma} \text { has no roots in the interval }\left(\lambda_{n}^{\prime}, \lambda_{n+1}^{\prime}\right) \\ 1 & \text { if } p^{\gamma} \text { has } 1 \text { root in }\left(\lambda_{n}^{\prime}, \lambda_{n+1}^{\prime}\right) \\ 0 & \text { if } p^{\gamma} \text { has } 2 \text { roots in }\left(\lambda_{n}^{\prime}, \lambda_{n+1}^{\prime}\right)\end{cases}
$$

Intervals having exponent $i_{n}=0$ are to be deleted from the product. Thus $\mathcal{R}_{\gamma} \subseteq$ $\mathbf{R}_{d_{\gamma}-1+\Delta_{\gamma}}$. Let $\mathcal{K}_{\gamma} \subseteq \mathbf{P}_{d_{\gamma}-1+\Delta}$ denote the positive cone over $\mathbf{p}\left[\mathcal{R}_{\gamma}\right]$. Let $\mathcal{A}_{\gamma} \subseteq$ $\mathbf{P}_{d_{\gamma}-1+\Delta}$ denote the affine subspace

$$
p^{\gamma}+p_{\gamma} \mathbf{P}_{\Delta_{\gamma}-1} .
$$


Proposition 3.9. The coordinate base $\mathcal{C}_{\gamma}$ is a semi-algebraic subset of $\mathbf{P}_{d_{\gamma}-1+\Delta_{\gamma}}$.

Proof. It is immediate from the definitions that

$$
\mathcal{C}_{\gamma}=\mathcal{K}_{\gamma} \cap \mathcal{A}_{\gamma} .
$$

$\mathcal{A}_{\gamma}$ is semi-algebraic, and semi-algebraic sets are closed under finite intersections; so it remains only to show that $\mathcal{K}_{\gamma}$ is semi-algebraic. Recall that we coordinatize $\mathbf{P}_{d_{\gamma}-1+\Delta_{\gamma}}$ by coefficients. Observe that $\mathcal{K}_{\gamma}$ is the image of $\mathbf{R}^{+} \times \mathcal{R}_{\gamma}$ by the map

$$
\left(r_{0}, r\right) \longmapsto r_{0} \mathbf{p}(r),
$$

the individual coordinates of which are polynomials. Now, it is a straightforward consequence of the Tarski-Seidenberg theorem [BR90] that the image of a semialgebraic set by a map each of whose coordinates is a polynomial, is semi-algebraic. The set $\mathbf{R}^{+} \times \mathcal{R}_{\gamma}$ is an open, convex polyhedron, and hence semi-algebraic. Therefore its image $\mathcal{K}_{\gamma}$ is semi-algebraic.

Every semi-algebraic set is a finite union of real-analytic manifolds [BR90]. The dimension of a semi-algebraic set is defined to be the dimension of its highestdimensional component manifold. It is in this sense that we refer to the dimension of $\mathcal{C}_{\gamma}$. Noting that $\mathbf{P}_{n}$ has dimension $n+1$, the fact that $\mathcal{C}_{\gamma} \subseteq \mathcal{A}_{\gamma}$ implies that $\operatorname{dim}\left(\mathcal{C}_{\gamma}\right) \leq \Delta_{\gamma}$. We show in the next subsection that, in general, $\operatorname{dim}\left(\mathcal{C}_{\gamma}\right)=\Delta_{\gamma}$. But first we make some observations about $\mathcal{K}_{\gamma}$ for future reference.

The map $\mathbf{p}$ is a diffeomorphism in a neighbourhood of any point $a \in \mathbf{R}^{n}$ that has distinct entries. In this connection, see BR90 Proposition 1.6.1]. It follows that $\mathbf{p}\left[\mathcal{R}_{\gamma}\right]$ has nonempty interior relative to the hyperplane in $\mathbf{P}_{d_{\gamma}-1+\Delta}$ consisting of those polynomials whose coefficient of $x^{d_{\gamma}-1+\Delta_{\gamma}}$ is $(-1)^{d_{\gamma}-1+\Delta_{\gamma}}$. The positive cone $\mathcal{K}_{\gamma}$ over $\mathbf{p}\left[\mathcal{R}_{\gamma}\right]$ therefore has nonempty interior relative to $\mathbf{P}_{d_{\gamma}-1+\Delta_{\gamma}}$. The set $\mathcal{K}_{\gamma}$ is, in general, neither open nor closed in $\mathbf{P}_{d_{\gamma}-1+\Delta_{\gamma}}$ (p is not injective on $\mathcal{R}_{\gamma}$ ). But it is straightforward to see that a point $q \in \partial \mathcal{K}_{\gamma}$ in the boundary of $\mathcal{K}_{\gamma}$ must have either a multiple root, or a root in $\Lambda^{\prime}=\left\{\lambda_{1}^{\prime}, \ldots, \lambda_{d-1}^{\prime}\right\}$.

3.4. Geometric properties of the coordinate base. A convex polyhedron in an affine space is usually defined to be the intersection of a finite number of closed half-spaces, and so is by definition closed. See $\mathrm{Br} \varnothing 83$. This is too restrictive for present considerations; so we relax the usual definition as follows. By a convex polyhedron we mean the intersection of a finite number of half spaces, each of which may be open or closed. With this definition, convex polyhedra are precisely the semi-algebraic sets that can be defined by a single system of first-degree polynomial inequalities. A bounded convex polyhedron is called a convex polytope.

We use two technical lemmas to derive the basic geometric properties of $\mathcal{C}_{\gamma}$. The first lemma uses a perturbation argument to construct a line segment in the relative interior of $\mathcal{C}_{\gamma}$. The second lemma concerns the geometry of the map

$$
r \longmapsto \mathbf{p}(r) \text {. }
$$

The idea behind the first lemma is that there exists a $\Delta_{\gamma}$-degree divisor $s_{0}$ of $p_{\gamma}$ such that every sufficiently small perturbation

$$
q_{\epsilon}=\epsilon p^{\gamma}+p_{\gamma} s_{0}
$$

of $p_{\gamma} s_{0}$ in the direction of $p^{\gamma}$ moves the roots of $p_{\gamma} s_{0}$ so that $q_{\epsilon}$ satisfies condition (i) of Definition 3.7. The difficulty lies in choosing $s_{0}$ correctly, which we now explain how to do. 
Notation. The index $i_{n}$ occurring in equation (28) serves to partition the $\gamma$-intervals $I_{n}=\left(\lambda_{n}, \lambda_{n+1}\right)$ into three sets:

$$
\begin{aligned}
& \mathcal{I}_{2}=\left\{I_{n} \mid i_{n}=2\right\} ; \\
& \mathcal{I}_{1}=\left\{I_{n} \mid i_{n}=1\right\} ; \\
& \mathcal{I}_{0}=\left\{I_{n} \mid i_{n}=0\right\} .
\end{aligned}
$$

Proposition 3.10. (i) $\left|\mathcal{I}_{2}\right|-\left|\mathcal{I}_{0}\right|=\Delta_{\gamma}$. (ii) The sets $\mathcal{I}_{2}$, $\mathcal{I}_{0}$ interlace, as follows: the left-most and right-most intervals in $\mathcal{I}_{2} \cup \mathcal{I}_{0}$ both belong to $\mathcal{I}_{2}$, and there is at least one member of $\mathcal{I}_{2}$ between any two consecutive members of $\mathcal{I}_{0}$.

Proof. This can be proved directly, as is done in Gib00, but it is more convenient to use some results appearing much later in the paper. A careful examination of the construction given in Section 4 reveals that, in general, there exists a regular solution $(\alpha, i, j) \in \Phi^{-1}([d \gamma])$ such that the roots of $q_{i}^{\alpha} q_{j}^{\alpha}$ are confined to the members of $\mathcal{I}_{0} \cup \mathcal{I}_{2}$, and moreover, such that $q_{i}^{\alpha}$ has precisely one root in each member of $\mathcal{I}_{0}$, and no root in any member of $\mathcal{I}_{2}$, while $q_{j}^{\alpha}$ has precisely one root in each member of $\mathcal{I}_{2}$, and no root in any member of $\mathcal{I}_{0}$. Therefore $\left|\mathcal{I}_{2}\right|-\left|\mathcal{I}_{0}\right|=j-i=\Delta_{\gamma}$. Part (ii) follows from the interlacing of $q_{i}^{\alpha}, q_{j}^{\alpha}$.

Notation. We can match each $I_{n} \in \mathcal{I}_{0}$ with the right-most member of $\mathcal{I}_{2}$ that lies to the left of $I_{n}$. In total, this pairs $\left|\mathcal{I}_{0}\right|$ members of $\mathcal{I}_{2}$ bijectively with the members of $\mathcal{I}_{0}$. Let $\mathcal{I}_{2}^{\prime} \subseteq \mathcal{I}_{2}$ denote the remaining members of $\mathcal{I}_{2}$, excluding the right-most member of $\mathcal{I}_{2}$. Thus, by Proposition 3.10 .

$$
\left|\mathcal{I}_{2}^{\prime}\right|=\Delta_{\gamma}-1
$$

We define the roots of $s_{0}$ to be the right endpoints of the members of $\mathcal{I}_{2}^{\prime}$, that is,

$$
s_{0}(x)=-\prod_{I_{n} \in \mathcal{I}_{2}^{\prime}}\left(\lambda_{n+1}^{\prime}-x\right) .
$$

If $\Delta_{\gamma}=1$, then $\mathcal{I}_{2}^{\prime}=\emptyset$. In this case, set $s_{0}(x)=-1$.

Note that interchanging left and right in the above description yields an alternative, and equally valid, choice for $s_{0}$.

Proposition 3.11. For $\epsilon>0$, define $q_{\epsilon}$ as in (29). For each $1 \leq n \leq d_{\gamma}-1$, set $\mu_{n}$ to be the midpoint of the $\gamma$-interval $\left(\lambda_{n}^{\prime}, \lambda_{n+1}^{\prime}\right)$. Set

$$
M=\min _{n}\left\{\left|q_{0}\left(\mu_{n}\right) / p^{\gamma}\left(\mu_{n}\right)\right|\right\} .
$$

Then, for every $0<\epsilon<M$ :

(i) $p^{\gamma} q_{\epsilon}$ has precisely two roots in each $\gamma$-interval;

(ii) $q_{\epsilon}$ has at most one root in each interval of the form $\left(\lambda_{n}^{\prime}, \mu_{n}\right),\left(\mu_{n}, \lambda_{n+1}^{\prime}\right)$, with none of the $\mu_{n}$ being a root of $q_{\epsilon}$.

The verification of this result requires a detailed and tedious analysis of sign patterns, which appears in full in Gib00. We omit it from the present exposition.

We come now to the first of our two lemmas. Let $M$ be defined as in Proposition 3.11 and set

$$
\widetilde{q}_{\epsilon}=\frac{1}{\epsilon} q_{\epsilon}, \quad L_{M}=\left\{\widetilde{q}_{\epsilon} \mid 0<\epsilon<M\right\} .
$$

Lemma 3.12. $L_{M} \subseteq \mathcal{C}_{\gamma} \cap \operatorname{int} \mathcal{K}_{\gamma}$. Furthermore,

$$
\left(\text { roots of } \widetilde{q}_{\epsilon}\right) \longrightarrow\left(\text { roots of } q_{0}=p_{\gamma} s_{0}\right) \text { as } \epsilon \longrightarrow 0 \text {. }
$$


Proof. The roots of $\widetilde{q}_{\epsilon}$ are the same as those of $q_{\epsilon}$, and evidently

$$
\text { (roots of } \left.q_{\epsilon}\right) \longrightarrow\left(\text { roots of } q_{0}\right) \text { as } \epsilon \longrightarrow 0 \text {. }
$$

Part (i) of Proposition 3.11 shows that $\widetilde{q}_{\epsilon} \in \mathcal{K}_{\gamma}$, and by construction $\widetilde{q}_{\epsilon}=p^{\gamma}+$ $\epsilon^{-1} p_{\gamma} s_{0} \in \mathcal{A}_{\gamma}$. Therefore $\widetilde{q}_{\epsilon} \in \mathcal{C}_{\gamma}$.

As mentioned earlier, boundary points $q \in \partial \mathcal{K}_{\gamma}$ are characterized as having either a multiple root, or a root in $\Lambda^{\prime}=\left\{\lambda_{1}^{\prime}, \ldots, \lambda_{d_{\gamma}}^{\prime}\right\}$. But Proposition 3.11 precludes both of these possibilities for $\widetilde{q}_{\epsilon} \in L_{M}$.

Let $l_{1}, \ldots, l_{m}$ and $r_{1}, \ldots, r_{m}$ satisfy the order relation

$$
l_{1}<r_{1} \leq l_{2}<r_{2} \leq \ldots \leq l_{m}<r_{m},
$$

and write

$$
\mathcal{R}=\prod_{n=1}^{m}\left[l_{n}, r_{n}\right]
$$

Proposition 3.13. Let $h \in \mathbf{P}_{m}$ have exact degree $m$, and suppose that the leading coefficient of $h$ has sign $(-1)^{m}$. Then the following conditions are equivalent:

(i) for each $n(1 \leq n \leq m),(-1)^{n} h\left(l_{n}\right) \leq 0$ and $(-1)^{n} h\left(r_{n}\right) \geq 0$;

(ii) $h$ has $m$ real roots $s_{1} \leq \ldots \leq s_{m}$ such that $s_{n} \in\left[l_{n}, r_{n}\right]$ for each $n$.

Proof. The equivalence of the following slightly modified conditions is straightforward: (i') for each $n(1 \leq n \leq m),(-1)^{n} h\left(l_{n}\right)<0$ and $(-1)^{n} h\left(r_{n}\right)>0$; (ii') $h$ has $m$ real roots $s_{1}<\ldots<s_{m}$ such that each $s_{n}$ is in the open interval $\left(l_{n}, r_{n}\right)$. The equivalence of the statements (i) and (ii) then follows by considering degree $m$ perturbations of $h$ and taking limits.

We call a polynomial

$$
p \in \mathbf{p}[\mathcal{R}]
$$

a vertex of $\mathbf{p}[\mathcal{R}]$ if $p=\mathbf{p}\left(a_{1}, \ldots, a_{m}\right)$ for some $\left(a_{1}, \ldots, a_{m}\right) \in \mathcal{R}$, where

$$
\left\{a_{1}, \ldots, a_{m}\right\} \subseteq\left\{l_{1}, \ldots, l_{m}, r_{1}, \ldots, r_{m}\right\} .
$$

Write $V$ for the set of all vertices of $\mathbf{p}[\mathcal{R}]$.

Lemma 3.14. $\mathbf{p}[\mathcal{R}]$ is a closed convex polytope in $\mathbf{P}_{m}$. Specifically, $\mathbf{p}[\mathcal{R}]$ is the convex hull of the finite set $V$.

Proof. Let $\operatorname{conv}(V)$ denote the convex hull of $V$. We show first that $\operatorname{conv}(V) \subseteq$ $\mathbf{p}[\mathcal{R}]$. Let $\sum t_{n} v_{n} \in \operatorname{conv}(V)$ be an arbitrary convex combination of vertices $v_{n} \in V$. Note that each vertex $v_{n}$ satisfies condition (ii) of Proposition 3.13 (with $h=v_{n}$ ), and hence condition (i) also. But condition (i) is easily seen to be invariant under positive combinations. That is, if condition (i) holds for $h=f$ and $h=g$, then condition (i) holds also for $h=s f+t g$ for any scalars $s, t>0$. Thus $h=\sum t_{n} v_{n}$ satisfies condition (i) and hence condition (ii) of Proposition 3.13 But condition (ii), together with the observation that the leading coefficient of $\sum t_{n} v_{n}$ is $(-1)^{m}$, implies that $\sum t_{n} v_{n}=\mathbf{p}(a)$ for some $a \in \mathcal{R}$.

We now argue the reverse inclusion $\mathbf{p}[\mathcal{R}] \subseteq \operatorname{conv}(V)$. We proceed by induction on $m$. If $m=1$, then $\mathbf{p}$ is linear, and the inclusion is obvious. So assume $k \geq 1$ and the desired inclusion holds for $m \leq k$. We will show that it holds for $m=$ $k+1$ also. Let $a=\left(a_{1}, \ldots, a_{k+1}\right) \in \prod_{n=1}^{k+1}\left[l_{n}, r_{n}\right]$ be arbitrary. Then $a_{k+1} \in$ $\left[l_{k+1}, r_{k+1}\right]$; so we may express $a_{k+1}$ as a convex combination of $l_{k+1}$ and $r_{k+1}$, say 
$a_{k+1}=(1-t) l_{k+1}+t r_{k+1}$. Also, $\left(a_{1}, \ldots, a_{k}\right) \in \prod_{n=1}^{k}\left[l_{n}, r_{n}\right]$; so by the inductive assumption, $\mathbf{p}\left(a_{1}, \ldots, a_{k}\right)=\sum t_{n} v_{n}$ for some convex combination of vertices $v_{n}$ of $\mathbf{p}\left[\prod_{n=1}^{k}\left[l_{n}, r_{n}\right]\right]$. Thus,

$$
\begin{aligned}
\mathbf{p} & \left(a_{1}, \ldots, a_{k+1}\right) \\
& =\mathbf{p}\left(a_{1}, \ldots, a_{k},(1-t) l_{k+1}+t r_{k+1}\right) \\
& =(1-t) \mathbf{p}\left(a_{1}, \ldots, a_{k}, l_{k+1}\right)+t \mathbf{p}\left(a_{1}, \ldots, a_{k}, r_{k+1}\right) \\
& =(1-t)\left(l_{k+1}-x\right) \mathbf{p}\left(a_{1}, \ldots, a_{k}\right)+t\left(r_{k+1}-x\right) \mathbf{p}\left(a_{1}, \ldots, a_{k}\right) \\
& =(1-t)\left(l_{k+1}-x\right)\left(\sum t_{n} v_{n}\right)+t\left(r_{k+1}-x\right)\left(\sum t_{n} v_{n}\right) \\
& =\sum\left((1-t) t_{n}\left(l_{k+1}-x\right) v_{n}+t t_{n}\left(r_{k+1}-x\right) v_{n}\right) .
\end{aligned}
$$

The latter is a convex combination of vertices $\left(l_{k+1}-x\right) v_{n}$ and $\left(r_{k+1}-x\right) v_{n}$ of $\mathbf{p}\left[\prod_{n=1}^{k+1}\left[l_{n}, r_{n}\right]\right]$. Thus $\mathbf{p}\left[\prod_{n=1}^{k+1}\left[l_{n}, r_{n}\right]\right]$ is contained in the convex hull of its vertices, as desired.

It is essential in the above lemma that the intervals $\left[l_{n}, r_{n}\right]$ do not overlap, except possibly at their endpoints. On the other hand, it is not essential that the intervals in terms of which $\mathcal{R}$ is defined be closed, in order for $\mathbf{p}[\mathcal{R}]$ to be a convex polytope. The lemma obviously generalizes as follows. Referring to the same intervals $\left[l_{n}, r_{n}\right]$ as before, let $I_{n}$ denote any one of $\left[l_{n}, r_{n}\right],\left[l_{n}, r_{n}\right),\left(l_{n}, r_{n}\right],\left(l_{n}, r_{n}\right)$, for $1 \leq n \leq m$. Set

$$
\mathcal{R}^{\prime}=\prod_{n=1}^{m} I_{n}
$$

Lemma 3.14'. $\mathbf{p}\left[\mathcal{R}^{\prime}\right]$ is a convex polytope in $\mathbf{P}_{m}$.

Theorem 3.15. The coordinate base $\mathcal{C}_{\gamma}$ is arcwise connected, as is int $\mathcal{C}_{\gamma}$, the interior of $\mathcal{C}_{\gamma}$ relative to $\mathcal{A}_{\gamma}$. Moreover, the closure of int $\mathcal{C}_{\gamma}$ includes $\mathcal{C}_{\gamma}$.

Proof. Let $q \in \mathcal{C}_{\gamma}$. We will show that there is a convex polyhedron $X_{q} \subseteq \mathcal{C}_{\gamma}$ such that, for sufficiently small $\epsilon, \widetilde{q}_{\epsilon} \in L_{M}$, and both $q$ and $\widetilde{q}_{\epsilon}$ are contained in $X_{q}$. It follows by convexity of $X_{q}$ that $\mathcal{C}_{\gamma}$ includes the line segment connecting $q$ and $\widetilde{q}_{\epsilon}$. Thus every $q \in \mathcal{C}_{\gamma}$ is connected to $L_{M}$ by a line segment, proving that $\mathcal{C}_{\gamma}$ is connected.

To construct $X_{q}$, choose a refinement of the collection of $\gamma$-intervals $I_{n}$ as follows. Break each interval $I_{n} \in \mathcal{I}_{2}$ (which contains two roots of $q$ ) into two subintervals

$$
I_{n}^{l}=\left(\lambda_{n}^{\prime}, \eta_{n}\right], \quad I_{n}^{r}=\left[\eta_{n}, \lambda_{n+1}^{\prime}\right),
$$

such that each subinterval contains one root of $q$. (Note that $q$ may have a double root $r$, in which case the definition requires $\eta_{n}=r$.) The domain

$$
\mathcal{R}_{q}=\prod_{I_{n} \in \mathcal{I}_{0} \cup \mathcal{I}_{1}} I_{n} \times \prod_{I_{n} \in \mathcal{I}_{2}}\left(I_{n}^{l} \times I_{n}^{r}\right)
$$

is contained in $\mathcal{R}_{\gamma}$. By Lemma[3.14, $\mathbf{p}\left[\mathcal{R}_{q}\right]$ is a convex polytope. Thus the positive cone $\mathcal{K}_{q}$ over $\mathbf{p}\left[\mathcal{R}_{q}\right]$ is a convex polyhedron.

Let int $\mathcal{K}_{q}$ denote the interior of $\mathcal{K}_{q}$ relative to $\mathbf{P}_{d_{\gamma}-1+\Delta_{\gamma}}$. Note that $\mathbf{p}$ is a diffeomorphism on the interior of $\mathcal{R}_{q}$ in $\mathbf{R}^{d_{\gamma}-1+\Delta_{\gamma}}$. See [BR90. Proposition 1.6.1]. Consequently, int $\mathcal{K}_{q}$ is an open subset of $\mathbf{P}_{d_{\gamma}-1+\Delta_{\gamma}}$. By the latter part of Lemma [3.12 
we can choose $\epsilon>0$ sufficiently small that $\widetilde{q}_{\epsilon} \in \operatorname{int} \mathcal{K}_{q}$. By construction the convex polyhedron

$$
\mathcal{K}_{q} \cap \mathcal{A}_{\gamma}
$$

includes both $\widetilde{q}_{\epsilon}$ and $q$, and is contained in $\mathcal{C}_{\gamma}$.

Let $S$ denote the line segment defined as the convex hull of $\left\{q, \widetilde{q}_{\epsilon}\right\}$. Then, since $\widetilde{q}_{\epsilon} \in \operatorname{int} \mathcal{K}_{q}$ and $\mathcal{K}_{q}$ is convex, the segment $S \backslash\{q\}$ lies within int $\mathcal{K}_{q}$. It also lies within $\mathcal{A}_{\gamma}$, and hence within $\operatorname{int} \mathcal{C}_{\gamma}$. It follows that int $\mathcal{C}_{\gamma}$ is connected. Moreover, since $q$ is in the closure of $S \backslash\{q\}$, the closure of int $\mathcal{C}_{\gamma}$ includes all of $\mathcal{C}_{\gamma}$.

Corollary 3.16. The dimension of $\mathcal{C}_{\gamma}$ is $\Delta_{\gamma}$.

Proof. The theorem implies that $\operatorname{int} \mathcal{C}_{\gamma}$ is nonempty. By definition, $\operatorname{int} \mathcal{C}_{\gamma}$ is a relatively open subset of the $\Delta_{\gamma}$-dimensional affine space $\mathcal{A}_{\gamma}$.

\section{Construction of the fibres}

This section is concerned with the construction of fibres $\rho^{-1}(X)$, where $X \in$ $\pi^{-1}([d \gamma])$ for some $[d \gamma] \in \operatorname{Ran} \Phi$. We will define the construction over $\{[d \gamma]\} \times \mathcal{C}_{\gamma}$, which by Proposition 3.8 includes $\pi^{-1}([d \gamma])$. The proof that every output of the construction is a solution (Theorem 5.8 ) will then imply that, in fact,

$$
\{[d \gamma]\} \times \mathcal{C}_{\gamma}=\pi^{-1}([d \gamma])
$$

(Theorem [5.10).

Letting $(\alpha, i, j) \in \Phi^{-1}([d \gamma])$, we pointed out at the end of Section 2.6 that a solution corresponding to known data $d \gamma$ can be constructed using the roots of the five polynomials

$$
p_{\alpha}, q_{i}^{\alpha}, q_{j}^{\alpha}, q_{d-1-i}^{\alpha^{f}}, q_{d-1-j}^{\alpha^{f}} .
$$

(Whether or not the solution so constructed is $(\alpha, i, j)$ itself depends on the particular choice of weights associated with jump points $\lambda_{n}$ of $\alpha$ at which $q_{i}^{\alpha}\left(\lambda_{n}\right)=$ $q_{j}^{\alpha}\left(\lambda_{n}\right)=0$, if any such jump points exist.) Two other polynomials are associated to the image

$$
\rho(\alpha, i, j)=\left([d \gamma], q^{(\alpha, i, j)}\right)
$$

of $(\alpha, i, j)$, namely,

$$
p^{\gamma}, q^{(\alpha, i, j)} .
$$

A detailed analysis of the location of the roots of the polynomials (31) relative to those of (32) underpins our construction of the fibre $\rho^{-1}\left([d \gamma], q^{(\alpha, i, j)}\right)$. We are able to say, given the roots of (32), precisely which collections $R$ of sets of reals are realizable as roots of the polynomials (31), for some $(\alpha, i, j) \in \rho^{-1}\left([d \gamma], q^{(\alpha, i, j)}\right)$.

The collections $R$ of roots serve as parameters, in terms of which solutions in $\rho^{-1}\left([d \gamma], q^{(\alpha, i, j)}\right)$ are constructed via the rational functions of Corollary 2.8.

4.1. Realizable patterns of roots. The following notation is to be fixed throughout the present section. Let $(\alpha, i, j) \in \mathcal{D}$ and let $d \gamma=q_{i}^{\alpha} q_{j}^{\alpha} d \alpha$. We are concerned here with the case $i<j$ corresponding to $\Delta_{\gamma}>0$. Let $\lambda_{1}<\ldots<\lambda_{d_{\alpha}}$ be the jump points of $\alpha$ and let $\lambda_{1}^{\prime}<\ldots<\lambda_{d_{\gamma}}^{\prime}$ be those of $\gamma$. Thus $\lambda_{1}^{\prime}, \ldots, \lambda_{d_{\gamma}}^{\prime}$ is the subsequence 
of $\lambda_{1}, \ldots, \lambda_{d_{\alpha}}$ obtained by deleting the points $\lambda_{n}$ at which $q_{i}^{\alpha}\left(\lambda_{n}\right) q_{j}^{\alpha}\left(\lambda_{n}\right)=0$. Writing $\alpha(x)=\sum_{n=1}^{d_{\alpha}} w_{n} H\left(x-\lambda_{n}\right)$, we have

$$
\begin{aligned}
\gamma(x) & =\sum_{n=1}^{d_{\alpha}} q_{i}^{\alpha}\left(\lambda_{n}\right) q_{j}^{\alpha}\left(\lambda_{n}\right) w_{n} H\left(x-\lambda_{n}\right) \\
& =\sum_{n=1}^{d_{\gamma}} q_{i}^{\alpha}\left(\lambda_{n}^{\prime}\right) q_{j}^{\alpha}\left(\lambda_{n}^{\prime}\right) w_{n} H\left(x-\lambda_{n}^{\prime}\right) .
\end{aligned}
$$

We explore the arrangement of the roots of $q_{i}^{\alpha} q_{j}^{\alpha}$ in an open $\gamma$-interval $\left(\lambda_{n}^{\prime}, \lambda_{n+1}^{\prime}\right)$. The interlacing of orthogonal polynomials narrowly restricts the possibilities.

Proposition 4.1. Suppose $q_{i}^{\alpha} q_{j}^{\alpha}$ has a multiple root $r$ in the interval $\left(\lambda_{n}^{\prime}, \lambda_{n+1}^{\prime}\right)$. Then the multiplicity of $r$ is exactly 2 , and $q_{i}^{\alpha} q_{j}^{\alpha}$ has no root in $\left(\lambda_{n}^{\prime}, \lambda_{n+1}^{\prime}\right)$ distinct from $r$.

Proof. That the multiplicity of $r$ is 2 follows immediately from the fact that $q_{i}$ and $q_{j}$ each have simple roots. Now, suppose that $q_{i}^{\alpha} q_{j}^{\alpha}$ has a root in $\left(\lambda_{n}^{\prime}, \lambda_{n+1}^{\prime}\right)$ distinct from $r$, and let $s \neq r$ be the root of $q_{i}^{\alpha} q_{j}^{\alpha}$ in $\left(\lambda_{n}^{\prime}, \lambda_{n+1}^{\prime}\right)$ closest to $r$. Then $s$ is a root of either $q_{i}^{\alpha}$ or $q_{j}^{\alpha}$. In either case, it follows from interlacing of the roots of $q_{i}^{\alpha}$ and $q_{j}^{\alpha}$ with those of $p_{\alpha}$ that there is a jump point $\lambda_{m}$ of $\alpha$ strictly between $r$ and $s$. But this implies that $\lambda_{m}$ is not a jump point of $\gamma$ (since $\lambda_{n}^{\prime}$ and $\lambda_{n+1}^{\prime}$ are consecutive jump points of $\gamma$, and $\lambda_{m}$ is between them). Therefore, $q_{i}^{\alpha}\left(\lambda_{m}\right) q_{j}^{\alpha}\left(\lambda_{m}\right)=0$. Yet $\lambda_{m}$ is closer to $r$ than $s$ is, contradicting the fact that $s$ is the closest root of $q_{i}^{\alpha} q_{j}^{\alpha}$ to $r$.

Proposition 4.2. Suppose that $q_{i}^{\alpha} q_{j}^{\alpha}$ has at least two distinct roots in the interval $\left(\lambda_{n}^{\prime}, \lambda_{n+1}^{\prime}\right)$, and list all such roots as $r_{1}<\ldots<r_{k}$. Then each of the roots is simple, and in the sequence $r_{1}, \ldots, r_{k}$, roots of $q_{i}^{\alpha}$ alternate with roots of $q_{j}^{\alpha}$ (i.e., there are never two consecutive roots of either $q_{i}^{\alpha}$ or $\left.q_{j}^{\alpha}\right)$. Moreover, for each $m$ in the range $2 \leq m \leq k-1$, the root $r_{m}$ is a jump point of $\alpha$.

Proof. That the roots are simple follows immediately from the preceding Proposition 4.1 Suppose that for some $m, r_{m}$ and $r_{m+1}$ are both roots of $q_{i}^{\alpha}$. Then by interlacing, the open interval $\left(r_{m}, r_{m+1}\right)$ contains a jump point of $\alpha$, which is necessarily a root of $q_{i}^{\alpha} q_{j}^{\alpha}$, a contradiction. Exactly the same reasoning shows that $r_{m}$ and $r_{m+1}$ are not both roots of $q_{j}^{\alpha}$. Therefore, roots of $q_{i}^{\alpha}$ and $q_{j}^{\alpha}$ alternate in the sequence $r_{1}, \ldots, r_{k}$, as desired.

Now, if $m$ is in the range $2 \leq m \leq k-1$, then $r_{m-1}$ and $r_{m+1}$ are both roots of $q_{i}^{\alpha}$ or both roots of $q_{j}^{\alpha}$. Therefore, by interlacing, the open interval $\left(r_{m-1}, r_{m+1}\right)$ contains a jump point of $\alpha$. This jump point has to be a root of $q_{i}^{\alpha} q_{j}^{\alpha}$. The only possibility is $r_{m}$, and so $r_{m}$ is a jump point of $\alpha$.

Proposition 4.3. Write $q=q^{(\alpha, i, j)}$ and let $r_{n} \leq r_{n}^{\prime}$ be the two roots of $p^{\gamma} q$ in the open interval $\left(\lambda_{n}^{\prime}, \lambda_{n+1}^{\prime}\right)$. Write $a=q_{i}^{\alpha} q_{d-1-i}^{\alpha^{f}}$ and $b=q_{j}^{\alpha} q_{d-1-j}^{\alpha^{f}}$. Then all the roots of ab in $\left(\lambda_{n}^{\prime}, \lambda_{n+1}^{\prime}\right)$ lie within the closed interval $\left[r_{n}, r_{n}^{\prime}\right]$, and if $r_{n}<r_{n}^{\prime}$, then, in the interval $\left[r_{n}, r_{n}^{\prime}\right]$, roots of a alternate with roots of $b$.

Proof. Suppose first that $r_{n}=r_{n}^{\prime}$, in which case the interval $\left[r_{n}, r_{n}^{\prime}\right]$ collapses to a single point $\left\{r_{n}\right\}$. If $r_{n}$ is a jump point of $\alpha$, then Lemma 3.1 (i) and the factorizations of $p^{\gamma}, q$ force $r_{n}$ to be a double root of both $q_{i}^{\alpha} q_{j}^{\alpha}$ and $q_{d-1-i}^{\alpha^{f}} q_{d-1-j}^{\alpha^{f}}$; Proposition 4.1 then implies that $a b$ has no root in $\left(\lambda_{n}^{\prime}, \lambda_{n}^{\prime}\right)$ different from $r_{n}$. If, 
on the other hand, $r_{n}$ is not a jump point of $\alpha$, then Lemma 3.1 (i) forces $r_{n}$ to be a simple root of both $a$ and $b$. Now, any root $s$ of $a b$ in $\left(\lambda_{n}^{\prime}, \lambda_{n+1}^{\prime}\right)$ different from $r_{n}$ must be a jump point of $\alpha$, since by assumption $s$ is not a root of $p^{\gamma} q$. Lemma 3.1 (i) then forces $s$ to be a double root of one of $a$ or $b$. Consider the closest such $s$ to $r_{n}$ and observe that Lemma 3.1(ii) is violated in the interval determined by the jump points of $\alpha$ lying on either side of $r_{n}$ (one of which is $s$ ), since $s$ is a double root of $a$ or $b$ while $r_{n}$ is a simple root of the same. Thus no such $s$ exists, meaning that $r_{n}$ is the only root of $a b$ in $\left(\lambda_{n}^{\prime}, \lambda_{n+1}^{\prime}\right)$.

Suppose next that $r_{n}<r_{n}^{\prime}$. In this case, neither $r_{n}$ nor $r_{n}^{\prime}$ can be a jump point of $\alpha$; for, if either of them is, say $r_{n}$, then the factorization of $p^{\gamma}, q$ and Lemma 3.1 (i) force $r_{n}$ to be a double root of both $q_{i}^{\alpha} q_{j}^{\alpha}$ and $q_{d-1-i}^{\alpha^{f}} q_{d-1-j}^{\alpha^{f}}$, and in this case Proposition 4.1 precludes the existence of $r_{n}^{\prime}$.

If $r_{n}, r_{n}^{\prime}$ are both roots of $q_{i}^{\alpha} q_{j}^{\alpha}$, or both roots of $q_{d-1-i}^{\alpha^{f}} q_{d-1-j}^{\alpha^{f}}$, then Proposition 4.2 applied to $\alpha$ or $\alpha^{f}$, respectively, shows that all the other roots of $a b$ in $\left(\lambda_{n}^{\prime}, \lambda_{n+1}^{\prime}\right)$ lie in $\left(r_{n}, r_{n}^{\prime}\right)$. But in any case, Proposition 4.2 shows that all the other roots of $a b$ within $\left(\lambda_{n}^{\prime}, \lambda_{n+1}^{\prime}\right)$ (all of which are jump points of $\alpha$ ) lie to one side only of each of $r_{n}$ and $r_{n}^{\prime}$. The only such arrangement consistent with Lemma 3.1 (ii) is for these other roots to lie between $r_{n}, r_{n}^{\prime}$, or for $r_{n}, r_{n}^{\prime}$ to both be roots of $q_{i}^{\alpha} q_{j}^{\alpha}$, or both roots of $q_{d-1-i}^{\alpha^{f}} q_{d-1-j}^{\alpha^{f}}$; the latter case was dealt with above. This completes the proof that all the roots of $a b$ in $\left(\lambda_{n}^{\prime}, \lambda_{n+1}^{\prime}\right)$ lie within the closed interval $\left[r_{n}, r_{n}^{\prime}\right]$.

It remains to argue that, in the case $r_{n}<r_{n}^{\prime}$, roots of $a$ alternate with roots of $b$ in $\left[r_{n}, r_{n}^{\prime}\right]$. If $r_{n}, r_{n}^{\prime}$ are both roots of $q_{i}^{\alpha} q_{j}^{\alpha}$, or both roots of $q_{d-1-i}^{\alpha^{f}} q_{d-1-j}^{\alpha^{f}}$, then Proposition 4.2, applied to $\alpha$ or $\alpha^{f}$ respectively, gives the desired result immediately. On the other hand, as long as there is at least one root of $a b$ in $\left(r_{n}, r_{n}^{\prime}\right)$, applying Proposition 4.2 to both $\alpha$ and $\alpha^{f}$ gives the desired result. Finally, if $r_{n}<r_{n}^{\prime}$ are the only roots of $a b$ in $\left[r_{n}, r_{n}^{\prime}\right]$, and one is a root of $q_{i}^{\alpha} q_{j}^{\alpha}$ while the other is a root of $q_{d-1-i}^{\alpha^{f}} q_{d-1-j}^{\alpha^{f}}$, then Lemma 3.1 (ii) prohibits that $r_{n}, r_{n}^{\prime}$ both be roots of $a$, or both be roots of $b$.

Proposition 4.4. Let $(\alpha, i, j) \in \mathcal{D}, q=q^{(\alpha, i, j)}, d=d_{\alpha}$ and $d \gamma=q_{i}^{\alpha} q_{j}^{\alpha} d \alpha$. Then the sets defined according to the left-hand side of the following table satisfy the characterization in terms of $p^{\gamma}, q$ given on the right-hand side of the table.

Proof. The proof is based on the formulas

$$
\begin{aligned}
p^{\gamma} & =-\left(b_{1} \cdots b_{d-1}\right) q_{i}^{\alpha} q_{d-1-j}^{\alpha^{f}} / p_{\alpha_{0}}, \\
q & =-\left(b_{1} \cdots b_{d-1}\right) q_{j}^{\alpha} q_{d-1-i}^{\alpha^{f}} / p_{\alpha_{0}}
\end{aligned}
$$

in conjunction with Lemma 3.1 As an example of the detailed argument involved, we prove that the sets $A_{1}, B_{1}^{f}$, as defined by the left-hand side of the table, satisfy the characterization given on the right-hand side, i.e.,

$$
A_{1} \cup B_{1}^{f}=S,
$$

where $S$ denotes the simple roots of $p^{\gamma}$ that are not roots of $q$. The inclusion $A_{1} \cup B_{1}^{f} \subseteq S$ is immediate from the definition of $A_{1}, B_{1}^{f}$ and (33). To see that $S \subseteq A_{1} \cup B_{1}^{f}$, let $r \in S$. Then by (33) $r$ must be a root of $q_{i}^{\alpha} q_{d-1-j}^{\alpha^{f}}$. Suppose $r$ is a root of $q_{i}^{\alpha}$. Since $r$ is simple root of $p^{\gamma}$, it must either be a root of both $q_{d-1-j}^{\alpha^{f}}$ and $p_{\alpha_{0}}$, or neither. In the former case, $r$ is a jump point of $\alpha$; so Lemma 3.1 (i) implies that $r$ is a root of both $q_{d-1-i}^{\alpha^{f}}$ and $q_{j}^{\alpha}$. But then by (33), $r$ is a root of $q$, a 


\begin{tabular}{|c|c|c|c|}
\hline \multirow[t]{2}{*}{ set } & \multicolumn{2}{|c|}{$\begin{array}{l}\text { definition in terms of } \\
q_{i}^{\alpha}, q_{j}^{\alpha}, q_{d-1-i}^{\alpha^{f}}, q_{d-1-j}^{\alpha^{f}}, p_{\alpha}\end{array}$} & \multirow[t]{2}{*}{$\begin{array}{l}\text { characterization } \\
\text { in terms of } p^{\gamma}, q\end{array}$} \\
\hline & $\begin{array}{l}\text { common roots of } \\
\text { the following } \\
\text { polynomials: }\end{array}$ & $\begin{array}{l}\text { not roots of } \\
\text { the following: }\end{array}$ & \\
\hline$A_{0}$ & $q_{i}^{\alpha}, q_{d-1-i}^{\alpha^{f}}, p_{\alpha}$ & $q_{j}^{\alpha}, q_{d-1-j}^{\alpha^{f}}$ & \multirow{2}{*}{$\begin{array}{l}\text { roots of neither } \\
p^{\gamma} \text { nor } q\end{array}$} \\
\hline$B_{0}$ & $q_{j}^{\alpha}, q_{d-1-j}^{\alpha^{f}}, p_{\alpha}$ & $q_{i}^{\alpha}, q_{d-1-i}^{\alpha^{f}}$ & \\
\hline$A_{1}$ & $q_{i}^{\alpha}$ & $q_{j}^{\alpha}, q_{d-1-i}^{\alpha^{f}}, q_{d-1-j}^{\alpha^{f}}, p_{\alpha}$ & \multirow{2}{*}{$\begin{array}{l}\text { simple roots of } \\
p^{\gamma} \text { that are not } \\
\text { roots of } q\end{array}$} \\
\hline$\overline{B_{1}^{f}}$ & $q_{d-1-j}^{\alpha^{f}}$ & $q_{i}^{\alpha}, q_{j}^{\alpha}, q_{d-1-i}^{\alpha^{f}}, p_{\alpha}$ & \\
\hline$B_{1}$ & $q_{j}^{\alpha}$ & $q_{i}^{\alpha}, q_{d-1-i}^{\alpha^{f}}, q_{d-1-j}^{\alpha^{f}}, p_{\alpha}$ & \multirow{2}{*}{$\begin{array}{l}\text { simple roots of } \\
q \text { that are not } \\
\text { roots of } p^{\gamma}\end{array}$} \\
\hline$A_{1}^{f}$ & $q_{d-1-i}^{\alpha^{f}}$ & $q_{i}^{\alpha}, q_{j}^{\alpha}, q_{d-1-j}^{\alpha^{f}}, p_{\alpha}$ & \\
\hline$C_{0}$ & $q_{i}^{\alpha}, q_{j}^{\alpha}, q_{d-1-i}^{\alpha^{f}}, q_{d-1-j}^{\alpha^{f}}, p_{\alpha}$ & & \multirow{3}{*}{$\begin{array}{l}\text { common roots } \\
\text { of } p^{\gamma} \text { and } q\end{array}$} \\
\hline$C_{1}$ & $q_{i}^{\alpha}, q_{j}^{\alpha}$ & $q_{d-1-i}^{\alpha^{f}}, q_{d-1-j}^{\alpha^{f}}, p_{\alpha}$ & \\
\hline$C_{1}^{f}$ & $q_{d-1-i}^{\alpha^{f}}, q_{d-1-j}^{\alpha^{f}}$ & $q_{i}^{\alpha}, q_{j}^{\alpha}, p_{\alpha}$ & \\
\hline$D$ & $q_{i}^{\alpha}, q_{d-1-j}^{\alpha^{f}}$ & $q_{j}^{\alpha}, q_{d-1-i}^{\alpha^{f}}, p_{\alpha}$ & double roots of $p^{\gamma}$ \\
\hline$D^{f}$ & $q_{j}^{\alpha}, q_{d-1-i}^{\alpha^{f}}$ & $q_{i}^{\alpha}, q_{d-1-j}^{\alpha^{f}}, p_{\alpha}$ & double roots of $q$ \\
\hline
\end{tabular}

contradiction. Hence $r$ is neither a root of $q_{d-1-j}^{\alpha^{f}}$ nor of $p_{\alpha_{0}}$. Since it is not a root of $q$, and not a root of $p_{\alpha_{0}}$, by (33) $r$ cannot be a root of $q_{d-1-i}^{\alpha^{f}}$ or of $q_{j}^{\alpha}$. Thus if $r$ is a root of $q_{i}^{\alpha}$, it is not a root of $q_{j}^{\alpha}, q_{d-1-i}^{\alpha^{f}}, q_{d-1-j}^{\alpha^{f}}$ or $p_{\alpha}$.

A parallel argument shows that if $r$ is a root of $q_{d-1-j}^{\alpha^{f}}$, it is not a root of $q_{i}^{\alpha}, q_{j}^{\alpha}, q_{d-1-i}^{\alpha^{f}}$ or $p_{\alpha}$, completing the proof that $S \subseteq A_{1} \cup B_{1}^{f}$.

Similar arguments justify the remainder of the table.

As stated earlier, the roots of $p_{\gamma}$ are denoted $\lambda_{1}^{\prime}, \ldots, \lambda_{d_{\gamma}}^{\prime}$.

Proposition 4.5. The sets defined according to the left-hand side of the table in Proposition 4.4 conform to the table below.

Proof. Applying Lemma 3.1 the sets defined in Proposition 4.4 account for all the roots of the polynomials

$$
q_{i}^{\alpha}, q_{j}^{\alpha}, q_{d-1-i}^{\alpha^{f}}, q_{d-1-j}^{\alpha^{f}},
$$

and for all the roots of $p_{\alpha}$ that are not roots of $p_{\gamma}$. The result then follows from the definitions of the sets in question.

4.2. Parametrization of the fibres. In addition to the notation fixed at the beginning of the section, we will use the following notation when dealing with a fixed member $q \in \mathcal{C}_{\gamma}$. 


\begin{tabular}{|c|c|}
\hline polynomial & roots \\
\hline \hline$q_{i}^{\alpha}$ & $A_{0} \cup A_{1} \cup C_{0} \cup C_{1} \cup D$ \\
\hline$q_{j}^{\alpha}$ & $B_{0} \cup B_{1} \cup C_{0} \cup C_{1} \cup D^{f}$ \\
\hline$q_{d-1-i}^{\alpha^{f}}$ & $A_{0} \cup A_{1}^{f} \cup C_{0} \cup C_{1}^{f} \cup D^{f}$ \\
\hline$q_{d-1-j}^{\alpha^{f}}$ & $B_{0} \cup B_{1}^{f} \cup C_{0} \cup C_{1}^{f} \cup D$ \\
\hline$p_{\alpha}$ & $A_{0} \cup B_{0} \cup C_{0} \cup\left\{\lambda_{1}^{\prime}, \ldots, \lambda_{d_{\gamma}}^{\prime}\right\}$ \\
\hline$p^{\gamma}$ & $A_{1} \cup B_{1}^{f} \cup C_{0} \cup C_{1} \cup C_{1}^{f} \cup D$ \\
\hline$q$ & $A_{1}^{f} \cup B_{1} \cup C_{0} \cup C_{1} \cup C_{1}^{f} \cup D^{f}$ \\
\hline
\end{tabular}

Notation. We know that there are precisely two roots of $p^{\gamma} q$ (counting multiplicity) in each interval $\left(\lambda_{n}^{\prime}, \lambda_{n+1}^{\prime}\right)$; let $r_{n} \leq r_{n}^{\prime}$ denote these two roots. We say that the interval $\left[r_{n}, r_{n}^{\prime}\right]$ is degenerate if $r_{n}=r_{n}^{\prime}$, or, equivalently, nondegenerate if $r_{n}<r_{n}^{\prime}$.

Given a polynomial $p$, let $\mathbf{s}(p)$ denote the set of simple roots of $p$, and let $\mathbf{d}(p)$ denote the set of roots of $p$ having multiplicity 2 (i.e., the double roots of $p$ ).

The eleven sets defined in Proposition 4.4 will serve to parametrize fibres over points $X \in \pi^{-1}([d \gamma]),[d \gamma] \in \operatorname{Ran} \Phi$. However, as mentioned earlier, our definitions will be given in terms of points in the coordinate base $\mathcal{C}_{\gamma}$.

Definition 4.6. We write $\operatorname{Cond}(\gamma, q, \mathcal{A})$ to indicate that the following set of conditions is satisfied by the triple $(\gamma, q, \mathcal{A})$. Firstly, $q \in \mathcal{C}_{\gamma}$, and

$$
\mathcal{A}=\left(A_{0}, A_{1}, A_{1}^{f}, B_{0}, B_{1}, B_{1}^{f}, C_{0}, C_{1}, C_{1}^{f}, D, D^{f}\right)
$$

is a sequence of eleven disjoint sets. We allow the possibility that sets occurring as entries of $\mathcal{A}$ may be empty. In addition:

1. $A_{1} \cup B_{1}^{f}=\mathbf{s}\left(p^{\gamma}\right) \backslash \mathbf{s}(q)$;

2. $B_{1} \cup A_{1}^{f}=\mathbf{s}(q) \backslash \mathbf{s}\left(p^{\gamma}\right)$;

3. $C_{0} \cup C_{1} \cup C_{1}^{f}=\mathbf{s}\left(p^{\gamma}\right) \cap \mathbf{s}(q)$;

4. $A_{0} \cup B_{0} \subseteq \bigcup_{n=1}^{d_{\gamma}-1}\left(r_{n}, r_{n}^{\prime}\right)$;

5. in each nondegenerate interval $\left[r_{n}, r_{n}^{\prime}\right]$, points of $A_{0} \cup A_{1} \cup A_{1}^{f}$ alternate with points of $B_{0} \cup B_{1} \cup B_{1}^{f}$;

6. $D=\mathbf{d}\left(p^{\gamma}\right)$;

7. $D^{f}=\mathbf{d}(q)$.

There are several remarks to be made about the above conditions. Construction of solutions to the main problem will involve choosing a sequence $\mathcal{A}$ so as to satisfy 1-7. Note that all of the available choice is captured in 1-4, with part 4 being constrained by condition 5 . Note further that selecting sets $A_{1}, B_{1}^{f}$, for example, simply entails choosing a partition of the set $\mathbf{s}\left(p^{\gamma}\right) \backslash \mathbf{s}(q)$ into two pieces, one of which is allowed to be empty. The situation for $B_{1}$, etc., is similar.

Definition 4.7. Given a sequence

$$
\mathcal{A}=\left(A_{0}, A_{1}, A_{1}^{f}, B_{0}, B_{1}, B_{1}^{f}, C_{0}, C_{1}, C_{1}^{f}, D, D^{f}\right),
$$

define a new sequence

$$
\mathcal{A}^{f}=\left(\widetilde{A}_{0}, \widetilde{A}_{1}, \widetilde{A}_{1}^{f}, \widetilde{B}_{0}, \widetilde{B}_{1}, \widetilde{B}_{1}^{f}, \widetilde{C}_{0}, \widetilde{C}_{1}, \widetilde{C}_{1}^{f}, \widetilde{D}, \widetilde{D}^{f}\right)
$$


in terms of $\mathcal{A}$ by setting

$$
\mathcal{A}^{f}=\left(B_{0}, B_{1}^{f}, B_{1}, A_{0}, A_{1}^{f}, A_{1}, C_{0}, C_{1}^{f}, C_{1}, D, D^{f}\right),
$$

so that $\widetilde{A}_{0}=B_{0}, \widetilde{A}_{1}=B_{1}^{f}$, and so forth. Call $\mathcal{A}^{f}$ the complementary sequence to $\mathcal{A}$. Henceforth we reserve the notation $\mathcal{A}^{f}$ for the complementary sequence to a sequence $\mathcal{A}$.

Observe that the conditions 1-7 in Definition 4.6] are invariant with respect to the map $\mathcal{A} \mapsto \mathcal{A}^{f}$. Therefore, $\operatorname{Cond}(\gamma, q, \mathcal{A})$ holds if and only if $\operatorname{Cond}\left(\gamma, q, \mathcal{A}^{f}\right)$ holds.

We now define the parameter domain in terms of which a fibre will be constructed.

Definition 4.8. For $q \in \mathcal{C}_{\gamma}$, let $\operatorname{Param}(\gamma, q)$ denote the set of pairs $(\mathcal{A}, c)$, such that:

1. $\operatorname{Cond}(\gamma, q, \mathcal{A})$ holds;

2. $c=\left(c_{r}\right)_{r \in C_{0}}$ is a finite sequence indexed over the entry $C_{0}$ of $\mathcal{A}$, and each $c_{r}>0$.

Thus each element of $\operatorname{Param}(\gamma, q)$ consists of a sequence $\mathcal{A}$ of sets and a possibly empty sequence $c$ of positive constants.

4.3. Construction of rational functions. We now describe in detail how to construct a solution $(\alpha, i, j) \in \Phi^{-1}([d \gamma])$ for each $q \in \mathcal{C}_{\gamma},(\mathcal{A}, c) \in \operatorname{Param}(\gamma, q)$.

The following consequence of the product rule for differentiation will be used repeatedly in what follows.

Observation 4.9. If $s \in S$ and $S, T$ are disjoint, then

$$
\left.\mathbf{p}^{\prime}(S \cup T)\right|_{x=s}=\left.\mathbf{p}^{\prime}(S) \mathbf{p}(T)\right|_{x=s} .
$$

Definition 4.10. We constructively define a map

$$
\Psi: \operatorname{Param}(\gamma, q) \longrightarrow \mathcal{D} \text {. }
$$

Let $(\mathcal{A}, c) \in \operatorname{Param}(\gamma, q)$. Referring explicitly to the entries of $\mathcal{A}$, construct the triple

$$
(\alpha, i, j)=\Psi(\mathcal{A}, c)
$$

as follows. Set

$$
\begin{aligned}
& f=-\frac{\mathbf{p}\left(A_{1}^{f} \cup C_{1}^{f} \cup D^{f}\right)}{\mathbf{p}^{\prime}\left(A_{0} \cup B_{0} \cup C_{0} \cup \Lambda^{\prime}\right) \mathbf{p}\left(A_{1} \cup C_{1} \cup D\right)} ; \\
& g=-\frac{\mathbf{p}\left(B_{1}^{f} \cup C_{1}^{f} \cup D\right)}{\mathbf{p}^{\prime}\left(A_{0} \cup B_{0} \cup C_{0} \cup \Lambda^{\prime}\right) \mathbf{p}\left(B_{1} \cup C_{1} \cup D^{f}\right)} ; \\
& h(x)=\max \left\{\frac{f(x)}{\sum_{n=1}^{d_{\gamma}} f\left(\lambda_{n}^{\prime}\right)}, \frac{g(x)}{\sum_{n=1}^{d_{\gamma}} g\left(\lambda_{n}^{\prime}\right)}\right\} ; \\
& \widetilde{\alpha}(x)=\sum_{r \in A_{0} \cup B_{0} \cup \Lambda^{\prime}} h(r) H(x-r)+\sum_{r \in C_{0}} c_{r} H(x-r) .
\end{aligned}
$$


Provided $\widetilde{\alpha} \in \mathcal{H}_{+}$(i.e., is positive), define $\alpha \in \mathcal{H}_{+, 1}$ by $\alpha \propto \widetilde{\alpha}$. Finally, set

$$
\begin{aligned}
i & =\left|A_{0} \cup A_{1} \cup C_{0} \cup C_{1} \cup D\right| ; \\
j & =\left|B_{0} \cup B_{1} \cup C_{0} \cup C_{1} \cup D^{f}\right| .
\end{aligned}
$$

This completes the construction of the triple $(\alpha, i, j)$.

Let $\operatorname{Constr}(\gamma, q)$ denote the image of $\operatorname{Param}(\gamma, q)$ by the map $\Psi$.

4.4. Properties of the construction. The next results establish some needed properties of the construction given in Definition 4.10.

Proposition 4.11. Both $f$ and $g$ are positive at points of $\Lambda^{\prime}$. For every $r \in A_{0}$, we have $g(r)>0, f(r)<0$, and for every $s \in B_{0}$, we have $f(s)>0, g(s)<0$.

Proof. Let $S$ denote the union of all the entries of $\mathcal{A}$ and write $p=\mathbf{p}\left(A_{0} \cup B_{0} \cup\right.$ $\left.C_{0} \cup \Lambda^{\prime}\right)$. Note that $S$ is contained in the interval $\left[\lambda_{1}^{\prime}, \lambda_{d_{\gamma}}^{\prime}\right]$. It follows immediately from the construction of $f, g$ that $f\left(\lambda_{1}^{\prime}\right)>0$ and $g\left(\lambda_{1}^{\prime}\right)>0$. Furthermore, we claim that for each $\lambda_{n}^{\prime}\left(n \leq d_{\gamma}-1\right), f$ and $g$ each change sign an even number of times from $\lambda_{n}^{\prime}$ to $\lambda_{n+1}^{\prime}$. To see this, consider the interval $I_{n}=\left(\lambda_{n}^{\prime}, \lambda_{n+1}^{\prime}\right)$, and as usual let $r_{n} \leq r_{n}^{\prime}$ denote the two roots of $p^{\gamma} q$ in $I_{n}$. If $r_{n}<r_{n}^{\prime}$, then the roots of $p^{\gamma} q$ in $I_{n}$ are simple, and so $r_{n}, r_{n}^{\prime} \in A_{1} \cup A_{1}^{\prime} \cup B_{1} \cup B_{1}^{\prime}$. The remaining points $r_{n}^{1}<\ldots<r_{n}^{k}$ of $S$ in $I_{n}$ (allowing $k=0$ ) are contained in $A_{0} \cup B_{0}$, and lie in the interval $\left(r_{n}, r_{n}^{\prime}\right)$. Now, the roots of $p$ in the closure of $I_{n}$ are $\lambda_{n}^{\prime}, r_{n}^{1}, \ldots, r_{n}^{k}, \lambda_{n+1}^{\prime}$; so $p^{\prime}$ has $k+1$ roots in $I_{n}$, one in each of the $k+1$ open intervals

$$
\left(\lambda_{n}^{\prime}, r_{n}^{1}\right),\left(r_{n}^{1}, r_{n}^{2}\right), \ldots,\left(r_{n}^{k-1}, r_{n}^{k}\right),\left(r_{n}^{k}, \lambda_{n+1}^{\prime}\right) .
$$

Note that, by condition 5 of Definition 4.6, the points $r_{n}^{1}<\ldots<r_{n}^{k}$ alternate between $A_{0}$ and $B_{0}$, and $k$ is odd precisely if $r_{n}$ and $r_{n}^{\prime}$ both belong to $A_{1} \cup A_{1}^{f}$, or both to $B_{1} \cup B_{1}^{f}$. (In this case, necessarily $k \geq 1$.) Thus $k$ is odd precisely if $A_{1} \cup A_{1}^{f}$, equivalently $B_{1} \cup B_{1}^{f}$, has an even number (0 or 2) of points in $I_{n}$. Otherwise $k$ is even and each of $A_{1} \cup A_{1}^{f}, B_{1} \cup B_{1}^{f}$ has a single point in $I_{n}$. Thus in any case, the sum of the $k+1$ roots of $p^{\prime}$, plus the number of points of $A_{1} \cup A_{1}^{f}$, or of $B_{1} \cup B_{1}^{f}$, in $I_{n}$, is even. It follows that each of $f$ and $g$ has an even number of sign changes in $I_{n}$, provided $r_{n}<r_{n}^{\prime}$.

If $r_{n}=r_{n}^{\prime}$, then $r_{n} \in C_{0} \cup C_{1} \cup C_{1}^{f} \cup D \cup D^{f}$ is the only point of $S$ in $I_{n}$. If $r_{n} \in C_{0}$, then $p^{\prime}$ has two roots in $I_{n}$; otherwise $p^{\prime}$ has a single root in $I_{n}$. In either case, by construction, $f, g$ each has precisely two sign changes in $I_{n}$. Therefore, each of $f, g$, being positive at $\lambda_{1}^{\prime}$, is positive at every point of $\Lambda^{\prime}$.

Note that $A_{0} \cup B_{0}$ has a point in $I_{n}$ only if $r_{n}<r_{n}^{\prime}$ and $k \geq 1$. Observe that in this case, $f$ changes sign twice or once in the interval $\left(\lambda_{n}^{\prime}, r_{n}^{1}\right)$, according as $r_{n} \in A_{1} \cup A_{1}^{f}$ or $r_{n} \in B_{1} \cup B_{1}^{f}$, respectively. Thus, according to condition 5 of Definition 4.6] $f\left(r_{n}^{1}\right)>0$ if and only if $r_{n} \in A_{1} \cup A_{1}^{f}$, if and only if $r_{n}^{1} \in B_{0}$. Since $f$ changes sign precisely once between successive points in the sequence $r_{n}^{1}, \ldots, r_{n}^{k}$, and because said sequence alternates between $A_{0}$ and $B_{0}$, it follows that $f\left(r_{n}^{i}\right)>0$ if and only if $r_{n}^{i} \in B_{0}$. Similarly, $g\left(r_{n}^{i}\right)>0$ if and only if $r_{n}^{i} \in A_{0}$. This completes the proof of the proposition.

It follows from the above proposition that the function $h$, as constructed in Definition 4.10, is strictly positive at each point of $A_{0} \cup B_{0} \cup C_{0} \cup \Lambda^{\prime}$, so that the corresponding distribution function $\widetilde{\alpha}$ is necessarily positive. Furthermore, at 
points of $B_{0}, h$ takes its value from (the normalized) $f$, and at points of $A_{0}, h$ takes its value from (the normalized) $g$. We will make use of this fact in later proofs.

Proposition 4.12. For every $\lambda_{i}^{\prime} \in \Lambda^{\prime}$,

$$
\left.\frac{f}{\sum_{n=1}^{d_{\gamma}} f\left(\lambda_{n}^{\prime}\right)}\right|_{x=\lambda_{i}^{\prime}}=\left.\frac{g}{\sum_{n=1}^{d_{\gamma}} g\left(\lambda_{n}^{\prime}\right)}\right|_{x=\lambda_{i}^{\prime}} .
$$

Proof. By construction, $f / g \propto q / p^{\gamma}$. By Definition 3.7 $q=p^{\gamma}\left(\bmod p_{\gamma}\right)$, and so it follows that

Since

$$
\frac{f}{\sum_{n=1}^{d_{\gamma}} f\left(\lambda_{n}^{\prime}\right)} \propto \frac{g}{\sum_{n=1}^{d_{\gamma}} g\left(\lambda_{n}^{\prime}\right)}\left(\bmod p_{\gamma}\right)
$$

$$
\sum_{i=1}^{d_{\gamma}} \frac{f\left(\lambda_{i}^{\prime}\right)}{\sum_{n=1}^{d_{\gamma}} f\left(\lambda_{n}^{\prime}\right)}=\sum_{i=1}^{d_{\gamma}} \frac{g\left(\lambda_{i}^{\prime}\right)}{\sum_{n=1}^{d_{\gamma}} g\left(\lambda_{n}^{\prime}\right)}=1,
$$

the constant of proportionality is 1 .

Proposition 4.13. If $(\alpha, i, j) \in \operatorname{Constr}(\gamma, q)$, then $j-i=\Delta_{\gamma}$.

Proof. Consider $I_{n}=\left(\lambda_{n}^{\prime}, \lambda_{n+1}^{\prime}\right)$, and suppose that $r_{n}<r_{n}^{\prime}$ are the two roots of $p^{\gamma} q$ in $I_{n}$. If $r_{n}, r_{n}^{\prime} \in \mathbf{s}(q)$, then conditions 1,2,4,5 of Definition 4.6 can be seen to imply that the set $A_{0} \cup A_{1}$ has one more point in $I_{n}$ than does $B_{0} \cup B_{1}$. If $r_{n}, r_{n}^{\prime} \in \mathbf{s}\left(p^{\gamma}\right)$, then $B_{0} \cup B_{1}$ has one more point in $I_{n}$ than $A_{0} \cup A_{1}$. If one of $r_{n}, r_{n}^{\prime}$ is a root of $p^{\gamma}$ and the other is a root of $q$, then the same conditions $1,2,4,5$ can be seen to imply that $A_{0} \cup A_{1}$ and $B_{0} \cup B_{1}$ have the same number of points in $I_{n}$.

In the case where $r_{n}=r_{n}^{\prime}$, if one of $r_{n}, r_{n}^{\prime}$ is a root of $p^{\gamma}$ and the other is a root of $q$, then $r_{n} \in C_{0} \cup C_{1} \cup C_{1}^{f}$. Otherwise $r_{n} \in D$, or $r_{n} \in D^{f}$. Based on the above observations, $j-i=\left|B_{0} \cup B_{1} \cup C_{0} \cup C_{1} \cup C_{1}^{f} \cup D^{f}\right|-\left|A_{0} \cup A_{1} \cup C_{0} \cup C_{1} \cup C_{1}^{f} \cup D\right|$ is precisely the difference between the number of $\gamma$-intervals containing 2 roots of $q$ and the number containing 2 roots of $p^{\gamma}$ (equivalently, no roots of $q$ ). By Proposition 3.10, this difference is precisely $\Delta \gamma$.

\section{MAIN THEOREMS}

\subsection{Every solution arises via the construction.}

Theorem 5.1. Let $(\alpha, i, j) \in \Phi^{-1}([d \gamma])$ and write $q=q^{(\alpha, i, j)}$. Then $q \in \mathcal{C}_{\gamma}$ and $(\alpha, i, j) \in \operatorname{Constr}(\gamma, q)$.

Proof. Note first that Theorem 3.2 and Proposition 3.6 combine to show that $q \in$ $\mathcal{C}_{\gamma}$. Define the entries of $\mathcal{A}$ in terms of the polynomials

$$
q_{i}^{\alpha}, q_{j}^{\alpha}, q_{d-1-i}^{\alpha^{f}}, q_{d-1-j}^{\alpha^{f}}, p_{\alpha}
$$

where $d=d_{\alpha}$, according to the left-hand side of Proposition 4.4. Then, by the right-hand side of Proposition 4.4. $\mathcal{A}$ satisfies properties 1,2,3,6,7 of Definition 4.6 By Proposition $4.3 \mathcal{A}$ satisfies properties 4,5 and thus $\operatorname{Cond}(\gamma, q, \mathcal{A})$ holds.

Next observe that, with $\mathcal{A}$ defined as above, the functions $f, g$ defined in Definition 4.10 can be written

$$
f=\frac{-\overline{q_{d-1-i}^{\alpha^{f}}}}{p_{\alpha}^{\prime} \overline{q_{i}^{\alpha}}}, \quad g=\frac{-\overline{q_{d-1-j}^{\alpha^{f}}}}{p_{\alpha}^{\prime} \overline{q_{j}^{\alpha}}} .
$$


Keeping $d=d_{\alpha}$, write $\alpha(x)=\sum_{n=1}^{d} w_{n} H\left(x-\lambda_{n}\right), \gamma(x)=\sum_{n=1}^{d_{\gamma}} w_{n}^{\prime} H\left(x-\lambda_{n}^{\prime}\right)$, so that

$$
\Lambda^{\prime}=\left\{\lambda_{1}^{\prime}, \ldots, \lambda_{d_{\gamma}}^{\prime}\right\} \subseteq\left\{\lambda_{1}, \ldots, \lambda_{d}\right\}=A_{0} \cup B_{0} \cup C_{0} \cup \Lambda^{\prime} .
$$

With this notation, Corollary 2.8 implies that, for $\lambda_{n} \in B_{0} \cup \Lambda^{\prime}$,

$$
w_{n}=\left(b_{1} \cdots b_{i}\right)^{2} f\left(\lambda_{n}\right),
$$

and for $\lambda_{n} \in A_{0} \cup \Lambda^{\prime}$,

$$
w_{n}=\left(b_{1} \cdots b_{j}\right)^{2} g\left(\lambda_{n}\right) .
$$

(Here the negatives of the scalars $b_{n}$ are next-to-diagonal entries of $J_{\alpha}$.)

Thus, setting $\nu=\sum_{\lambda_{n} \in \Lambda^{\prime}} w_{n}$, we have

$$
\nu=\left(b_{1} \cdots b_{i}\right)^{2} \sum_{n=1}^{d_{\gamma}} f\left(\lambda_{n}^{\prime}\right)=\left(b_{1} \cdots b_{j}\right)^{2} \sum_{n=1}^{d_{\gamma}} g\left(\lambda_{n}^{\prime}\right) .
$$

Using Proposition 4.11, it follows that $h$, as constructed in Definition 4.10, satisfies the relation $h\left(\lambda_{n}\right)=w_{n} / \nu$, for each $\lambda_{n} \in A_{0} \cup B_{0} \cup \Lambda^{\prime}$. For each $\lambda_{n} \in C_{0}$, define $c_{\lambda_{n}}=w_{n} / \nu$. Then $\widetilde{\alpha}$, as constructed in Definition 4.10, is precisely $\frac{1}{\nu} \alpha$. So, after normalization, the construction produces the $\alpha$ hypothesized in the theorem.

It remains to verify that

$$
i=\left|A_{0} \cup A_{1} \cup C_{0} \cup C_{1} \cup D\right| \text { and } j=\left|B_{0} \cup B_{1} \cup C_{0} \cup C_{1}\right|,
$$

but this is immediate from the way $\mathcal{A}$ was defined in terms of $(\alpha, i, j)$ via Proposition 4.4

\subsection{Some technical results.}

Proposition 5.2. Suppose $\Psi(\mathcal{A}, c)=(\alpha, i, j)$. Then, for an appropriate choice of constants $c^{f}, \Psi\left(\mathcal{A}^{f}, c^{f}\right)=\left(\alpha^{f}, d_{\alpha}-1-j, d_{\alpha}-1-i\right)$.

Proof. Write $\left(\alpha^{F}, \widetilde{\imath}, \widetilde{\jmath}\right)=\Psi\left(\mathcal{A}^{f}, c^{f}\right)$. Let $d_{\gamma}=d_{\gamma}$ (as before) and $d=d_{\alpha}\left(=d_{\alpha^{F}}\right)$. As in the definition of $\mathcal{A}^{f}$, let the entries of $\mathcal{A}^{f}$ be denoted with tildes to distinguish them from the corresponding entries of $\mathcal{A}$. We wish to show that for an appropriate choice of $c^{f}$ we have $\alpha^{F}=\alpha^{f}, \widetilde{\imath}=d-1-j$ and $\tilde{\jmath}=d-1-i$. In fact, the values of $\widetilde{\imath}, \tilde{\jmath}$ do not depend on $c^{f}$, as follows. According to Definition 4.10

$$
\begin{aligned}
i & =\left|A_{0} \cup A_{1} \cup C_{0} \cup C_{1} \cup D\right|, \\
\widetilde{\imath} & =\left|\widetilde{A}_{0} \cup \widetilde{A}_{1} \cup \widetilde{C}_{0} \cup \widetilde{C}_{1} \cup \widetilde{D}\right| \\
& =\left|B_{0} \cup B_{1}^{f} \cup C_{0} \cup C_{1}^{f} \cup D\right| .
\end{aligned}
$$

Thus,

$$
\begin{aligned}
i+\widetilde{\imath} & =\left|A_{0} \cup B_{0} \cup C_{0}\right|+\left|A_{1} \cup B_{1}^{f}\right|+\left|C_{0} \cup C_{1} \cup C_{1}^{f}\right|+2|D| \\
& =d-d_{\gamma}+\left|\mathbf{s}\left(p^{\gamma}\right) \backslash \mathbf{s}(q)\right|+\left|\mathbf{s}\left(p^{\gamma}\right) \cap \mathbf{s}(q)\right|+2\left|\mathbf{d}\left(p^{\gamma}\right)\right| \\
& =d-d_{\gamma}+\operatorname{deg}\left(p^{\gamma}\right) \\
& =d-d_{\gamma}+d_{\gamma}-1-\Delta_{\gamma} \\
& =d-1-j+i . \quad \text { (Note: } \Delta_{\gamma}=j-i \text { by Prop. 4.13) }
\end{aligned}
$$

Hence $\tilde{\imath}=d-1-j$, as desired. The argument that $\tilde{\jmath}=d-1-i$ is similar; alternatively, one can appeal to Proposition 4.13. 
We need to distinguish the objects arising in the construction of $\alpha^{F}$ from the corresponding objects for $\alpha$. Thus in the construction of $\alpha^{F}$, let $\widetilde{f}, \widetilde{g}, \widetilde{h}, \widetilde{\widetilde{\alpha}}$ denote the objects corresponding to $f, g, h, \widetilde{\alpha}$ in the construction of $\alpha$. Write

$$
p=\mathbf{p}\left(A_{0} \cup B_{0} \cup C_{0} \cup \Lambda^{\prime}\right)=\mathbf{p}\left(\widetilde{A_{0}} \cup \widetilde{B_{0}} \cup \widetilde{C_{0}} \cup \Lambda^{\prime}\right),
$$

so that $p=p_{\alpha}=p_{\alpha^{F}}$. Next we claim that

$$
\frac{1}{\sum_{n=1}^{d_{\gamma}} f\left(\lambda_{n}^{\prime}\right) \widetilde{g}\left(\lambda_{n}^{\prime}\right)}=\frac{1}{\sum_{n=1}^{d_{\gamma}} g\left(\lambda_{n}^{\prime}\right) \widetilde{f}\left(\lambda_{n}^{\prime}\right)} .
$$

To see this, recall that for every $\lambda_{n}^{\prime} \in \Lambda^{\prime}$,

$$
f\left(\lambda_{n}^{\prime}\right) / \sum_{n=1}^{d_{\gamma}} f\left(\lambda_{n}^{\prime}\right)=g\left(\lambda_{n}^{\prime}\right) / \sum_{n=1}^{d_{\gamma}} g\left(\lambda_{n}^{\prime}\right) ;
$$

and similarly,

$$
\widetilde{f}\left(\lambda_{n}^{\prime}\right) / \sum_{n=1}^{d_{\gamma}} \widetilde{f}\left(\lambda_{n}^{\prime}\right)=\widetilde{g}\left(\lambda_{n}^{\prime}\right) / \sum_{n=1}^{d_{\gamma}} \widetilde{g}\left(\lambda_{n}^{\prime}\right) .
$$

By the latter two equations,

$$
\frac{f\left(\lambda_{n}^{\prime}\right) \widetilde{g}\left(\lambda_{n}^{\prime}\right)}{\sum_{n=1}^{d_{\gamma}} f\left(\lambda_{n}^{\prime}\right) \widetilde{g}\left(\lambda_{n}^{\prime}\right)}=\frac{g\left(\lambda_{n}^{\prime}\right) \widetilde{f}\left(\lambda_{n}^{\prime}\right)}{\sum_{n=1}^{d_{\gamma}} g\left(\lambda_{n}^{\prime}\right) \widetilde{f}\left(\lambda_{n}^{\prime}\right)} .
$$

Now observe that $\left.f\left(\lambda_{n}^{\prime}\right) \widetilde{g}\left(\lambda_{n}^{\prime}\right)=g\left(\lambda_{n}^{\prime}\right) \widetilde{f}\left(\lambda_{n}^{\prime}\right)=\left(p^{\prime}\left(\lambda_{n}^{\prime}\right)\right)^{-2}\right)$, which implies the claim.

To show that $\alpha^{F}=\alpha^{f}$, it suffices to verify that $p^{\alpha} p^{\alpha^{F}} \propto 1(\bmod p)$. (See Proposition 3.3 ) Indeed, it is sufficient to verify that $p^{\widetilde{\alpha}} p^{\widetilde{\widetilde{\alpha}}} \propto 1(\bmod p)$. Thus, let $r$ be an arbitrary root of $p$, equivalently jump point of $\alpha$ and $\alpha^{F}$. Then, by Definition 4.10 and Proposition 4.11.

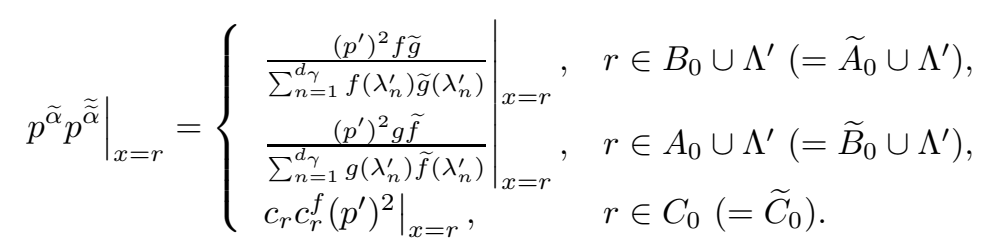

It is easily checked via Definition 4.10 that $\left(p^{\prime}\right)^{2} f \widetilde{g}=\left(p^{\prime}\right)^{2} g \widetilde{f}=1$. From the identity

$$
\frac{1}{\sum_{n=1}^{d_{\gamma}} f\left(\lambda_{n}^{\prime}\right) \widetilde{g}\left(\lambda_{n}^{\prime}\right)}=\frac{1}{\sum_{n=1}^{d_{\gamma}} g\left(\lambda_{n}^{\prime}\right) \widetilde{f}\left(\lambda_{n}^{\prime}\right)},
$$

it then follows that

$$
\left.p^{\widetilde{\alpha}} p^{\tilde{\widetilde{\alpha}}}\right|_{x=r}=\frac{1}{\sum_{n=1}^{d_{\gamma}} f\left(\lambda_{n}^{\prime}\right) \widetilde{g}\left(\lambda_{n}^{\prime}\right)},
$$

provided that $c_{r}^{f}$ is prescribed the value $\frac{c_{r}}{\left(p^{\prime}(r)\right)^{2} \sum_{n=1}^{d_{\gamma}} f\left(\lambda_{n}^{\prime}\right) \widetilde{g}\left(\lambda_{n}^{\prime}\right)}$ (necessarily positive) for each $r \in C_{0}$.

Proposition 5.3. If $r \in A_{0}$, then

$$
\left.\frac{q_{i} q_{j} g}{r-x}\right|_{x=r}=-\left.\frac{\overline{p^{\gamma}}}{p_{\gamma}}\right|_{x=r} .
$$


Proof. Note that, by definition of $q_{i}, q_{j}, g$,

$$
\begin{aligned}
& \frac{q_{i} q_{j} g}{r-x} \\
& =\frac{\mathbf{p}\left(\left(A_{0} \backslash\{r\}\right) \cup A_{1} \cup C_{0} \cup C_{1} \cup D\right) \mathbf{p}\left(B_{0} \cup B_{1} \cup C_{0} \cup C_{1} \cup D^{f}\right) \mathbf{p}\left(B_{1}^{f} \cup C_{1}^{f} \cup D\right)}{\mathbf{p}^{\prime}\left(A_{0} \cup B_{0} \cup C_{0} \cup \Lambda^{\prime}\right) \mathbf{p}\left(B_{1} \cup C_{1} \cup D^{f}\right)} .
\end{aligned}
$$

Since $r \in A_{0}$, it follows from Observation 4.9 that

$$
\left.\mathbf{p}^{\prime}\left(A_{0} \cup B_{0} \cup C_{0} \cup \Lambda^{\prime}\right)\right|_{x=r}=-\left.\mathbf{p}\left(\left(A_{0} \backslash\{r\}\right) \cup B_{0} \cup C_{0} \cup \Lambda^{\prime}\right)\right|_{x=r} .
$$

Substituting this in the denominator of the above expression and then cancelling like terms yields

$$
\begin{aligned}
\left.\frac{q_{i} q_{j} g}{r-x}\right|_{x=r} & =-\left.\frac{\mathbf{p}\left(A_{1} \cup B_{1}^{f} \cup C_{0} \cup C_{1} \cup C_{1}^{f} \cup D\right) \mathbf{p}(D)}{\mathbf{p}\left(\Lambda^{\prime}\right)}\right|_{x=r} \\
& =-\left.\frac{\overline{p^{\gamma}}}{p_{\gamma}}\right|_{x=r} .
\end{aligned}
$$

This completes the proof.

\section{Proposition 5.4.}

$$
\sum_{n=1}^{d_{\gamma}}\left(\left.\frac{q_{i} q_{j} g}{r-x}\right|_{x=\lambda_{n}^{\prime}}\right)=\left.\frac{\overline{p^{\gamma}}}{p_{\gamma}}\right|_{x=r} .
$$

Proof. By Observation 4.9 ,

$$
\begin{aligned}
\left.\mathbf{p}^{\prime}\left(A_{0} \cup B_{0} \cup C_{0} \cup \Lambda^{\prime}\right)\right|_{x=\lambda_{n}^{\prime}} & =\left.\mathbf{p}\left(A_{0} \cup B_{0} \cup C_{0}\right) \mathbf{p}^{\prime}\left(\Lambda^{\prime}\right)\right|_{x=\lambda_{n}^{\prime}} \\
& =\left.\mathbf{p}\left(A_{0} \cup B_{0} \cup C_{0}\right) p_{\gamma}^{\prime}\right|_{x=\lambda_{n}^{\prime}} .
\end{aligned}
$$

Substituting this into the right-hand side of

$$
\begin{aligned}
& \frac{q_{i} q_{j} g}{r-x} \\
& =\frac{\mathbf{p}\left(A_{0} \cup A_{1} \cup C_{0} \cup C_{1} \cup D\right) \mathbf{p}\left(B_{0} \cup B_{1} \cup C_{0} \cup C_{1} \cup D^{f}\right) \mathbf{p}\left(B_{1}^{f} \cup C_{1}^{f} \cup D\right)}{(r-x) \mathbf{p}^{\prime}\left(A_{0} \cup B_{0} \cup C_{0} \cup \Lambda^{\prime}\right) \mathbf{p}\left(B_{1} \cup C_{1} \cup D^{f}\right)},
\end{aligned}
$$

and then cancelling like terms, yields

$$
\begin{aligned}
\left.\frac{q_{i} q_{j} g}{r-x}\right|_{x=\lambda_{n}^{\prime}} & =\left.\frac{\mathbf{p}\left(A_{1} \cup B_{1}^{f} \cup C_{0} \cup C_{1} \cup C_{1}^{f} \cup D\right) \mathbf{p}(D)}{(r-x) p_{\gamma}^{\prime}}\right|_{x=\lambda_{n}^{\prime}} \\
& =\left.\frac{\overline{p^{\gamma}}}{(r-x) p_{\gamma}^{\prime}}\right|_{x=\lambda_{n}^{\prime}} .
\end{aligned}
$$


Let $c<0$ be the constant such that $\overline{p^{\gamma}}=c p^{\gamma}$. Then we have

$$
\begin{aligned}
\sum_{n=1}^{d_{\gamma}}\left(\left.\frac{q_{i} q_{j} g}{r-x}\right|_{x=\lambda_{n}^{\prime}}\right) & =c \sum_{n=1}^{d_{\gamma}}\left(\left.\frac{p^{\gamma}}{(r-x) p_{\gamma}^{\prime}}\right|_{x=\lambda_{n}^{\prime}}\right) \\
& \left.=c \int_{-\infty}^{\infty} \frac{d \gamma}{r-x} \quad \text { (by definition of } p^{\gamma}\right) \\
& =\left.c \frac{p^{\gamma}}{p_{\gamma}}\right|_{x=r}(\text { cf. proof of Lemma 2.2) } \\
& =\left.\frac{p^{\gamma}}{p_{\gamma}}\right|_{x=r} .
\end{aligned}
$$

This completes the proof.

Proposition 5.5. Let $q \in \mathcal{C}_{\gamma}$, and let a be a polynomial of degree $\Delta_{\gamma}$ that divides q. Then, for any $z \in \mathbf{C}$,

$$
\int_{-\infty}^{\infty} \frac{d \gamma}{a(z-x)}=\left.\frac{q}{a p_{\gamma}}\right|_{x=z}
$$

Proof. Note that $q$, and hence $a$, has no common zero with $p_{\gamma}$. Define $\widetilde{\gamma}$ by $d \widetilde{\gamma}=$ $d \gamma / a$. Then $p_{\widetilde{\gamma}}=p_{\gamma}$ and

$$
\int_{-\infty}^{\infty} \frac{d \gamma}{a(z-x)}=\int_{-\infty}^{\infty} \frac{d \widetilde{\gamma}}{z-x}=\left.\frac{p^{\tilde{\gamma}}}{p_{\gamma}}\right|_{x=z}
$$

the latter equality following from the proof of Lemma 2.2. Now, for each jump point $\lambda$ of $\gamma$ we have $q(\lambda)=p^{\gamma}(\lambda)$, and so

$$
p^{\widetilde{\gamma}}(\lambda)=\frac{p^{\gamma}(\lambda)}{a(\lambda)}=\frac{q(\lambda)}{a(\lambda)} .
$$

Since the degrees of $p^{\widetilde{\gamma}}$ and $q / a$ are both less than $d_{\gamma}$, it follows that $p^{\widetilde{\gamma}}=q / a$. Therefore,

$$
\int_{-\infty}^{\infty} \frac{d \gamma}{a(z-x)}=\left.\frac{q}{a p_{\gamma}}\right|_{x=z}
$$

as desired.

Proposition 5.6. Let a be a polynomial of degree $\Delta_{\gamma}$ that divides $q$ and has no root in $B_{0}$. If $r \in B_{0}$, then

$$
\left.\frac{q_{i} q_{j} f}{a(r-x)}\right|_{x=r}=-\left.\frac{\bar{q}}{a p_{\gamma}}\right|_{x=r} .
$$

Proof. Note that, by definition of $q_{i}, q_{j}, f$,

$$
\begin{aligned}
& \frac{q_{i} q_{j} f}{a(r-x)} \\
& =\frac{\mathbf{p}\left(A_{0} \cup A_{1} \cup C_{0} \cup C_{1} \cup D\right) \mathbf{p}\left(\left(B_{0} \backslash\{r\}\right) \cup B_{1} \cup C_{0} \cup C_{1} \cup D^{f}\right) \mathbf{p}\left(A_{1}^{f} \cup C_{1}^{f} \cup D^{f}\right)}{a \mathbf{p}^{\prime}\left(A_{0} \cup B_{0} \cup C_{0} \cup \Lambda^{\prime}\right) \mathbf{p}\left(A_{1} \cup C_{1} \cup D\right)} .
\end{aligned}
$$

Since $r \in B_{0}$, it follows from Observation 4.9 that

$$
\left.\mathbf{p}^{\prime}\left(A_{0} \cup B_{0} \cup C_{0} \cup \Lambda^{\prime}\right)\right|_{x=r}=-\left.\mathbf{p}\left(A_{0} \cup\left(B_{0} \backslash\{r\}\right) \cup C_{0} \cup \Lambda^{\prime}\right)\right|_{x=r} .
$$


Substituting this into the denominator of the above expression and then cancelling like terms yields

$$
\begin{aligned}
\left.\frac{q_{i} q_{j} f}{a(r-x)}\right|_{x=r} & =-\left.\frac{\mathbf{p}\left(A_{1}^{f} \cup B_{1} \cup C_{0} \cup C_{1} \cup C_{1}^{f} \cup D^{f}\right) \mathbf{p}\left(D^{f}\right)}{a \mathbf{p}\left(\Lambda^{\prime}\right)}\right|_{x=r} \\
& =-\left.\frac{\bar{q}}{a p_{\gamma}}\right|_{x=r},
\end{aligned}
$$

which completes the proof.

Proposition 5.7. Let a be a polynomial of degree $\Delta_{\gamma}$ that divides $q$ and has no root in $B_{0}$. Then

$$
\sum_{n=1}^{d_{\gamma}}\left(\left.\frac{q_{i} q_{j} f}{a(r-x)}\right|_{x=\lambda_{n}^{\prime}}\right)=\left.\frac{\bar{q}}{a p_{\gamma}}\right|_{x=r} .
$$

Proof. By Observation 4.9,

$$
\begin{aligned}
\left.\mathbf{p}^{\prime}\left(A_{0} \cup B_{0} \cup C_{0} \cup \Lambda^{\prime}\right)\right|_{x=\lambda_{n}^{\prime}} & =\left.\mathbf{p}\left(A_{0} \cup B_{0} \cup C_{0}\right) \mathbf{p}^{\prime}\left(\Lambda^{\prime}\right)\right|_{x=\lambda_{n}^{\prime}} \\
& =\left.\mathbf{p}\left(A_{0} \cup B_{0} \cup C_{0}\right) p_{\gamma}^{\prime}\right|_{x=\lambda_{n}^{\prime}} .
\end{aligned}
$$

Substituting this into the right-hand side of

$$
\begin{aligned}
& \frac{q_{i} q_{j} f}{a(r-x)} \\
& =\frac{\mathbf{p}\left(A_{0} \cup A_{1} \cup C_{0} \cup C_{1} \cup D\right) \mathbf{p}\left(B_{0} \cup B_{1} \cup C_{0} \cup C_{1} \cup D^{f}\right) \mathbf{p}\left(A_{1}^{f} \cup C_{1}^{f} \cup D^{f}\right)}{a(r-x) \mathbf{p}^{\prime}\left(A_{0} \cup B_{0} \cup C_{0} \cup \Lambda^{\prime}\right) \mathbf{p}\left(A_{1} \cup C_{1} \cup D\right)},
\end{aligned}
$$

and then cancelling like terms, yields

$$
\begin{aligned}
\left.\frac{q_{i} q_{j} f}{a(r-x)}\right|_{x=\lambda_{n}^{\prime}} & =\left.\frac{\mathbf{p}\left(A_{1}^{f} \cup B_{1} \cup C_{0} \cup C_{1} \cup C_{1}^{f} \cup D^{f}\right) \mathbf{p}\left(D^{f}\right)}{a(r-x) p_{\gamma}^{\prime}}\right|_{x=\lambda_{n}^{\prime}} \\
& =\left.\frac{\bar{q}}{a(r-x) p_{\gamma}^{\prime}}\right|_{x=\lambda_{n}^{\prime}} .
\end{aligned}
$$

Let $c<0$ be the constant such that $\bar{q}=c q$. Then we have

$$
\begin{aligned}
\sum_{n=1}^{d_{\gamma}}\left(\left.\frac{q_{i} q_{j} f}{a(r-x)}\right|_{x=\lambda_{n}^{\prime}}\right) & =c \sum_{n=1}^{d_{\gamma}}\left(\left.\frac{q}{a(r-x) p_{\gamma}^{\prime}}\right|_{x=\lambda_{n}^{\prime}}\right) \\
& =c \int_{-\infty}^{\infty} \frac{d \gamma}{a(r-x)} \quad\left(\text { since } q=p^{\gamma}\left(\bmod p_{\gamma}\right)\right) \\
& =\left.c \frac{q}{a p_{\gamma}}\right|_{x=r} \quad \text { (by Prop. 5.5) } \\
& =\left.\frac{\bar{q}}{a p_{\gamma}}\right|_{x=r} .
\end{aligned}
$$

This completes the proof. 


\subsection{Every output of the construction is a solution.}

Theorem 5.8. For every $q \in \mathcal{C}_{\gamma}$, Constr $(\gamma, q) \subseteq \Phi^{-1}([d \gamma])$.

Proof. Let $q \in \mathcal{C}_{\gamma},(\alpha, i, j) \in \operatorname{Constr}(\gamma, q)$. This means that $(\alpha, i, j)=\Psi(\mathcal{A}, c)$ for some $(\mathcal{A}, c) \in \operatorname{Param}(\gamma, q)$. (We use the notation in Definition 4.6 for the entries of $\mathcal{A}$.) We wish to prove that $\Psi(\mathcal{A}, c) \in \Phi^{-1}([d \gamma])$. Without loss of generality, we may assume $\left|B_{1} \cup C_{0} \cup C_{1} \cup D^{f}\right| \geq \Delta_{\gamma}$. (Otherwise, we can consider $\left(\mathcal{A}^{f}, c^{f}\right)$.) Referring to the entries of $\mathcal{A}$, define

$$
\begin{aligned}
q_{i} & =\mathbf{p}\left(A_{0} \cup A_{1} \cup C_{0} \cup C_{1} \cup D\right) ; \\
q_{j} & =\mathbf{p}\left(B_{0} \cup B_{1} \cup C_{0} \cup C_{1} \cup D^{f}\right) .
\end{aligned}
$$

We will show that:

1. $q_{i} q_{j} d \alpha \propto d \gamma$

2. $q_{j} \propto q_{j}^{\alpha}$

3. $q_{i} \propto q_{i}^{\alpha}$.

1. $q_{i} q_{j} d \alpha \propto d \gamma$. Observe that since $A_{0} \cup B_{0} \cup C_{0}$ are roots of $q_{i} q_{j}$, the distribution

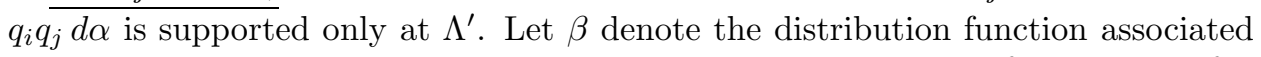
to the distribution $q_{i} q_{j} d \alpha$. By Proposition 4.11, the amplitude of the jumps of $\alpha$ at points of $\Lambda^{\prime}$ are proportional to both of the functions $f, g$ (where $f, g$ are the functions occurring in Definition 4.10). Thus, up to proportionality, the coefficients of $\beta$ have the form $q_{i}\left(\lambda_{n}^{\prime}\right) q_{j}\left(\lambda_{n}^{\prime}\right) g\left(\lambda_{n}^{\prime}\right)$. Writing out $q_{i}, q_{j}, g$ in terms of their defining sets of roots, one obtains

$$
\left(p_{\gamma}\right)^{\prime} q_{i} q_{j} g \propto p^{\gamma} \quad\left(\bmod p_{\gamma}\right)
$$

from which it follows, by definition of $p^{\gamma}$, that $q_{i} q_{j} d \alpha \propto d \gamma$.

2. $q_{j} \propto q_{j}^{\alpha}$. Note that $q_{j}$ has degree $j$. Thus to show $q_{j} \propto q_{j}^{\alpha}$, it suffices to show that for every $p \in \mathbf{P}_{j-1}, \int_{-\infty}^{\infty} p q_{j} d \alpha=0$. Toward this end, we choose a basis for $\mathbf{P}_{j-1}$ as follows. For each root $r$ of $q_{i}$, define $p_{r}=q_{i} /(r-x)$. Then the polynomials $p_{r}$, together with $q_{i}, x q_{i}, \ldots, x^{\Delta_{\gamma}-1} q_{i}$ span $\mathbf{P}_{j-1}$. (Recall $j-i=\Delta_{\gamma}$.) So we will show that for each polynomial in this spanning set, the integral in question is 0 . To begin, let $0 \leq n<\Delta_{\gamma}$ and consider $I_{n}=\int_{-\infty}^{\infty}\left(x^{n} q_{i}\right) q_{j} d \alpha$. By 1 , we have $I_{n} \propto \int_{-\infty}^{\infty} x^{n} d \gamma=0$, since $n<\Delta_{\gamma}$. It remains to verify that for each root $r$ of $q_{i}$, $I_{r}=\int_{-\infty}^{\infty} p_{r} q_{j} d \alpha$ is 0 . Observe that if $r \in A_{0}$, then $p_{r} q_{j} d \gamma=q_{i} q_{j} d \alpha /(r-x)$ is supported at $r$ and the points in $\Lambda^{\prime}$; otherwise, it is supported only at $\Lambda^{\prime}$. Note also that, by Proposition 4.11, the coefficients of $\alpha$ corresponding to points in $A_{0} \cup \Lambda^{\prime}$ are proportional to $g$ evaluated on the same set. Thus we have two cases. If $r \notin A_{0}$, then

$$
\begin{aligned}
\int_{-\infty}^{\infty} p_{r} q_{j} d \alpha & \left.\propto \sum_{n=1}^{d_{\gamma}} \frac{q_{i} q_{j} g}{r-x}\right|_{x=\lambda_{n}^{\prime}} \\
& =\left.\frac{\overline{p^{\gamma}}}{p_{\gamma}}\right|_{x=r},
\end{aligned}
$$


by Proposition 5.4. But every root $r$ of $q_{i}$ not in $A_{0}$ is a root of $p^{\gamma}$; so the above integral is 0 . If on the other hand $r \in A_{0}$, then

$$
\begin{aligned}
\int_{-\infty}^{\infty} p_{r} q_{j} d \alpha & \left.\propto \frac{q_{i} q_{j} g}{r-x}\right|_{x=r}+\sum_{n=1}^{d_{\gamma}}\left(\left.\frac{q_{i} q_{j} g}{r-x}\right|_{x=\lambda_{n}^{\prime}}\right) \\
& =-\left.\frac{\overline{p^{\gamma}}}{p_{\gamma}}\right|_{x=r}+\left.\frac{\overline{p^{\gamma}}}{p_{\gamma}}\right|_{x=r} \\
& =0,
\end{aligned}
$$

by Propositions 5.3 and 5.4. Thus $\int_{-\infty}^{\infty} p q_{j} d \alpha=0$ for every $p \in \mathbf{P}_{j-1}$, whereby $q_{i} \propto q_{i}^{\alpha}$.

3. $q_{i} \propto q_{i}^{\alpha}$. Here we make essential use of the assumption $\left|B_{1} \cup C_{0} \cup C_{1} \cup D^{f}\right|$

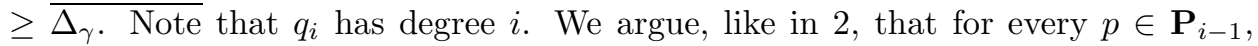
$\int_{-\infty}^{\infty} p q_{i} d \alpha=0$. We choose a basis of $\mathbf{P}_{i-1}$ as follows. Let $a$ be a divisor of $q_{j}$ having degree $\Delta_{\gamma}$ and no roots in $B_{0}$. Then, for each root $r$ of $q_{j}$ which is not a root of $a$, define $p_{r}$ to be the polynomial $\frac{q_{j}}{a(r-x)}$. There are $i$ such polynomials, and they span $\mathbf{P}_{i-1}$. Note that, over $B_{0} \cup \Lambda^{\prime}$, the coefficients of $\alpha$ are proportional to $f$, by Proposition 4.11 There are two cases. If $r \notin B_{0}$, then the distribution $p_{r} q_{j} d \gamma$ is supported only at $\Lambda^{\prime}$. In this case,

$$
\begin{aligned}
\int_{-\infty}^{\infty} p_{r} q_{i} d \alpha & \propto \sum_{n=1}^{d_{\gamma}}\left(\left.\frac{q_{i} q_{j} f}{a(r-x)}\right|_{x=\lambda_{n}^{\prime}}\right) \\
& =\left.\frac{\bar{q}}{a p_{\gamma}}\right|_{x=r},
\end{aligned}
$$

by Proposition 5.7 By definition of $q_{j}$, since $r$ is a root of $q_{j}$ and not in $B_{0}, r$ is necessarily a root of $q$, and the integral in question is 0 . If on the other hand $r \in B_{0}$, then the distribution $p_{r} q_{i} d \alpha$ is supported at $r$ and at the points of $\Lambda^{\prime}$. In this case,

$$
\begin{aligned}
\int_{-\infty}^{\infty} p_{r} q_{i} d \alpha & \left.\propto \frac{q_{i} q_{j} f}{a(r-x)}\right|_{x=r}+\sum_{n=1}^{d_{\gamma}}\left(\left.\frac{q_{i} q_{j} f}{a(r-x)}\right|_{x=\lambda_{n}^{\prime}}\right) \\
& =-\left.\frac{\bar{q}}{a p_{\gamma}}\right|_{x=r}+\left.\frac{\bar{q}}{a p_{\gamma}}\right|_{x=r} \\
& =0,
\end{aligned}
$$

by Propositions 5.6 and 5.7. This proves that $\int_{-\infty}^{\infty} p q_{i} d \alpha=0$ for every $p \in \mathbf{P}_{i-1}$, whereby $q_{i} \propto q_{i}^{\alpha}$.

The statement of the theorem follows immediately from $1,2,3$.

Proposition 5.9. If $(\alpha, i, j)=\Psi(\mathcal{A}, c)$, for $(\mathcal{A}, c) \in \operatorname{Param}(\gamma, q)$, then $(\alpha, i, j)$ satisfies Proposition 4.5 with respect to the entries of $\mathcal{A}$.

Proof. It was shown in the proof of Theorem 5.8 that $q_{i}^{\alpha}, q_{j}^{\alpha}$ conform to Proposition 4.5, under the assumption that at least $\Delta_{\gamma}$ roots of $q_{j}$ lie outside the set $B_{0}$. Definition 4.6 implies directly that $p^{\gamma}, q$ conform to Proposition4.5, Definition 4.10 implies that $p_{\alpha}$ conforms. To prove that $q_{d-1-j}^{\alpha^{f}}$ conforms, consider $\left(\mathcal{A}^{f}, c^{f}\right)$, for any $c^{f}$, and proceed as for $q_{i}^{\alpha}$ in the proof of Theorem 5.8 
Finally, to show that the roots of $q_{d-1-i}^{\alpha^{f}}$ are as specified in Proposition 4.5, we appeal to Proposition 2.7 and Definition 2.1 by which $-q_{d-1-i}^{\alpha^{f}} \propto q_{i}^{\alpha} p^{\alpha}$. Note that at points $r \in A_{0}$ we have $q_{i}^{\alpha}(r)=0$, so that

$$
q_{i}^{\alpha} p^{\alpha} \propto \overline{q_{i}^{\alpha}} p_{\alpha}^{\prime} f \quad\left(\bmod p_{\alpha}\right)
$$

Since $-q_{d-1-i}^{\alpha^{f}} \propto q_{i}^{\alpha} p^{\alpha}$, the desired decomposition of $q_{d-1-i}^{\alpha^{f}}$ follows from substituting the known expansions for $\overline{q_{i}^{\alpha}}, p_{\alpha}^{\prime}, f$.

Theorem 5.10. For every $[d \gamma] \in \operatorname{Ran} \Phi$ we have $\pi^{-1}([d \gamma])=\{[d \gamma]\} \times \mathcal{C}_{\gamma}$.

Proof. Proposition 5.9 implies, in particular, that $q \in \mathcal{C}_{\gamma}$ and $(\alpha, i, j) \in \operatorname{Constr}(\gamma, q)$ imply $q^{(\alpha, i, j)}=q$. Therefore, $\operatorname{Constr}(\gamma, q) \neq \emptyset$ and

$$
\{[d \gamma]\} \times \mathcal{C}_{\gamma} \subseteq \pi^{-1}([d \gamma])
$$

which is the reverse inclusion to Proposition 3.8

Theorem 5.11. For each $q \in \mathcal{C}_{\gamma}$, the map $\Psi: \operatorname{Param}(\gamma, q) \longrightarrow \operatorname{Constr}(\gamma, q)$ is injective (and hence bijective).

Proof. Suppose that $(\alpha, i, j)=\Psi(\mathcal{A}, c)=\Psi\left(\mathcal{A}^{\prime}, c^{\prime}\right)$. We need to show that $(\mathcal{A}, c)=$ $\left(\mathcal{A}^{\prime}, c^{\prime}\right)$. By Proposition [5.9, Proposition 4.5 is valid with respect to $(\alpha, i, j)$ and both $\mathcal{A}$ and $\mathcal{A}^{\prime}$. In terms of the polynomials $q_{i}^{\alpha}, q_{j}^{\alpha}$, etc., occurring in the left-hand side of Proposition 4.5, one can recover the individual entries of $\mathcal{A}$, and also $\mathcal{A}^{\prime}$, using the left-hand side of Proposition 4.4 Therefore $\mathcal{A}=\mathcal{A}^{\prime}$, since their entries are prescribed by the same polynomials. Given that $\mathcal{A}=\mathcal{A}^{\prime}$, it is evident from Definition 4.10 that the equality $\Psi(\mathcal{A}, c)=\Psi\left(\mathcal{A}^{f}, c^{f}\right)$ implies $c=c^{f}$.

\section{Illustration of the RESUlts}

We have proven that the map

$$
\Psi: \operatorname{Param}(\gamma, q) \longrightarrow \operatorname{Constr}(\gamma, q)
$$

is a bijection, and that

$$
\Phi^{-1}([d \gamma])=\bigcup_{q \in \mathcal{C}_{\gamma}} \operatorname{Constr}(\gamma, q)
$$

Why is this useful? Firstly, it enables one to construct solutions explicitly and to generate formulas for solutions. We give an example of this in Section 6.2. Secondly, the structure of $\operatorname{Param}(\gamma, q)$ is easy to analyse using Definition 4.6, and from this structure one can read off information about $\Phi^{-1}([d \gamma])$, such as Theorems 6.1 and 6.2 below.

6.1. Existence theorems. This subsection is based on an analysis of Definition 4.6. Because we have considered the general case, Definition 4.6 is somewhat complicated. Along with the map $\Psi$, it simplifies considerably in the generic situation where $p^{\gamma}$ and $q$ have only simple roots, and no root in common. Then each of the sets $C_{0}, C_{1}, C_{1}^{f}, D, D^{f}$ is empty, as is the sequence $c$ of Definition 4.8. On the other hand, common roots of $p^{\gamma}$ and $q$, and double roots of $p^{\gamma}$ and $q$, do occur. (It is easy to cook up examples.)

Suppose that $\operatorname{Cond}(\gamma, q, \mathcal{A})$ holds and each of $A_{0}, B_{0}, C_{0}$ is empty, and let $(\alpha, i, j)$ $=\Psi(\mathcal{A}, c)$, where necessarily $c=\emptyset$. Then Definition 4.10 shows that $\alpha$ has the same jump points as $\gamma$, so that $(\alpha, i, j)$ is a regular solution. Moreover, regular solutions 
are characterized by $A_{0}, B_{0}, C_{0}$ being empty. It is not a priori evident that regular solutions exist in general. But they do.

Theorem 6.1. For every $[d \gamma] \in \operatorname{Ran} \Phi$, the solution set $\Phi^{-1}([d \gamma])$ contains a $\Delta_{\gamma^{-}}$ dimensional manifold of regular solutions.

Proof. By Corollary 3.16, it suffices to show that for every $q \in \mathcal{C}_{\gamma}$, there exists an $\mathcal{A}$ such that $A_{0}=B_{0}=C_{0}=\emptyset$ and $\operatorname{Cond}(\gamma, q, \mathcal{A})$ holds. The partitions of $\mathbf{s}\left(p^{\gamma}\right) \backslash \mathbf{s}(q)$ and $\mathbf{s}(q) \backslash \mathbf{s}\left(p^{\gamma}\right)$ in 1, 2 of Definition 4.6 cannot be chosen arbitrarily. To satisfy 5 , in each nondegenerate $\gamma$-interval one of the two roots $r_{n}, r_{n}^{\prime}$ of $p^{\gamma} q$ must belong to $A_{1} \cup A_{1}^{f}$ and the other to $B_{1} \cup B_{1}^{f}$. But this choice can be made in exactly two ways per nondegenerate interval, and so is always possible. (There are three cases to consider: $r_{n}, r_{n}^{\prime}$ are both roots of $p^{\gamma}$, both of $q$, or one of each.)

The reasoning in the above proof can be pursued further to give a more precise description of the set of regular solutions. See Gib00.

Concerning singular solutions, we have the following result.

Theorem 6.2. Let $[d \gamma] \in \operatorname{Ran} \Phi$ and $\Delta_{\gamma}>0$. Then for every $d>d_{\gamma}$, there exists a solution $(\alpha, i, j) \in \Phi^{-1}([d \gamma])$ such that $d_{\alpha}=d$.

Proof. By Lemma 3.12, it is possible to select $q \in \mathcal{C}_{\gamma}$ with roots arbitrarily close to the jump points of $\gamma$. In particular, there exists a $q \in \mathcal{C}_{\gamma}$ such that each $\gamma$-interval $I_{n}$ containing a root of $q$ is nondegenerate, i.e., such that the two roots of $p^{\gamma} q$ in $I_{n}$ are distinct. In this case, $\mathbf{s}\left(p^{\gamma}\right) \cap \mathbf{s}(q)=\mathbf{d}(q)=\emptyset$.

Let $r_{n}<r_{n}^{\prime}$ be the two roots of $p^{\gamma} q$ in a fixed nondegenerate $\gamma$-interval $I_{n}$. Referring to Definition 4.6, partition those roots of $p^{\gamma} q$ in each nondegenerate interval different from $I_{n}$ such that roots in $A_{1} \cup A_{1}^{f}$ alternate with those in $B_{1} \cup B_{1}^{f}$, as in the proof of Theorem 6.1.

Let $d>d_{\gamma}$ be given. If $d-d_{\gamma}$ is even, assign one of $r_{n}, r_{n}^{\prime}$ to $A_{1} \cup A_{1}^{f}$ and the other to $B_{1} \cup B_{1}^{f}$. (This is always possible.) Then choose $d-d_{\gamma}$ points in the open interval $\left(r_{n}, r_{n}^{\prime}\right)$, and assign these, and only these, alternately to $A_{0}, B_{0}$ such that condition 5 of Definition 4.6 is satisfied. This determines a sequence $\mathcal{A}$ such that $\operatorname{Cond}(\gamma, q, \mathcal{A})$ holds and

$$
\left|A_{0} \cup B_{0} \cup C_{0}\right|=\left|A_{0} \cup B_{0}\right|=d-d_{\gamma} .
$$

Therefore $(\alpha, i, j)=\Psi(\mathcal{A}, c)$, where $c=\emptyset$, satisfies $d_{\alpha}=d$.

If $d-d_{\gamma}$ is odd, assign $r_{n}, r_{n}^{\prime}$ both to $A_{1} \cup A_{1}^{f}$ or both to $B_{1} \cup B_{1}^{f}$. Again, this is always possible. Then proceed as in the previous case.

The kind of analysis of Definition 4.6 used in the proofs of the above theorems can also be used to study the local dimension of $\Phi^{-1}([d \gamma])$, for instance.

6.2. An explicit formula. The main construction, given in Definition 4.10, can be used to generate explicit formulas. Here we work out an example. Let

$$
\gamma(x)=\frac{1}{2} H(x+1)-\frac{1}{2} H(x-1) .
$$

Note that $d \gamma=q_{0}^{\beta} q_{1}^{\beta} d \beta$, where

$$
\beta(x)=\frac{1}{2} H(x+1)+\frac{1}{2} H(x-1),
$$

and so $[d \gamma] \in \operatorname{Ran} \Phi$. 
We will choose a particular $q \in \mathcal{C}_{\gamma}$ and construct a sequence

$$
\left\{\left(\alpha_{d},\lfloor d / 2\rfloor-1,\lfloor d / 2\rfloor\right)\right\}_{d \geq 2}
$$

in the fibre $\rho^{-1}([d \gamma], q)$ in which $\alpha_{d}$ has $d$ jump points.

The composite polynomial of $\gamma$ works out to be a constant, $p^{\gamma}=-1$. Definition 3.7 characterizes $q \in \mathcal{C}_{\gamma}$ by:

1. $q$ has two roots, both in $(-1,1)$;

2. $q(-1)=q(1)=-1$.

It follows that the realizable roots of $q \in \mathcal{C}_{\gamma}$ are

$$
\{-1+t, 1-t\}, \quad 0<t \leq 1 \text {. }
$$

We choose $q \in \mathcal{C}_{\gamma}$ corresponding to $t=1 / 2$,

$$
q(x)=-\frac{4}{3}\left(x+\frac{1}{2}\right)\left(x-\frac{1}{2}\right) .
$$

(Of course it is enough to know the roots of $q$, the coefficients being irrelevant to the construction.) The set $\operatorname{Param}(\gamma, q)$ is determined by the various sets of roots of $p^{\gamma}$ and $q$, most of which are empty:

$$
\begin{aligned}
& \mathbf{s}\left(p^{\gamma}\right)=\mathbf{d}\left(p^{\gamma}\right)=\mathbf{d}(q)=\emptyset \\
& \mathbf{s}(q)=\{-1 / 2,1 / 2\}
\end{aligned}
$$

By Definitions 4.6 and 4.8 it follows that for any $(\mathcal{A}, c) \in \operatorname{Param}(\gamma, q)$, the sets

$$
A_{1}, B_{1}^{f}, C_{0}, C_{1}, C_{1}^{f}, D, D^{f}
$$

are empty, as is the sequence $c$. Constructing $(\mathcal{A}, c) \in \operatorname{Param}(\gamma, q)$ thus involves only the sets $B_{1}, A_{1}^{f}, A_{0}, B_{0}$, and parts $2,4,5$ of Definition 4.6.

We construct solutions $\left(\alpha_{d},\lfloor d / 2\rfloor-1,\lfloor d / 2\rfloor\right)$ in two groups, according to whether $d$ is odd or even. We consider odd $d$ first.

Let $d=2 n+3, n \geq 0$. Set $A_{1}^{f}=\{-1 / 2,1 / 2\}$ and $B_{1}=\emptyset$. Set

$$
x_{k}=-\frac{1}{2}+\frac{k+1}{2 n+2}, \quad k=0, \ldots, 2 n,
$$

and set

$$
\begin{aligned}
& A_{0}=\left\{x_{k} \mid k \text { is odd }\right\} \\
& B_{0}=\left\{x_{k} \mid k \text { is even }\right\}
\end{aligned}
$$

The sequence $\mathcal{A}$ defined according to the above values of $A_{1}^{f}, B_{1}, A_{0}, B_{0}$, and with the sets (36) being empty, satisfies $\operatorname{Cond}(\gamma, q, \mathcal{A})$. Therefore $(\mathcal{A}, \emptyset) \in \operatorname{Param}(\gamma, q)$.

The corresponding solution $\Psi(\mathcal{A}, \emptyset)$ is constructed explicitly in Definition 4.10 Substituting in the relevant sets and evaluating the corresponding rational functions at the points

$$
A_{0} \cup B_{0} \cup\{-1,1\}
$$


yields, after some simplification, the formula

$$
\begin{aligned}
\alpha_{2 n+3}(x) \propto & \frac{n !}{(3 n+2) !}\{H(x+1)+H(x-1)\} \\
& +\sum_{k=0}^{2 n} \frac{4\left((k+1)(2 n-k+1) /\left(3(n+1)^{2}\right)\right)^{\frac{1+(-1) k}{2}}}{(n+k+2)(3 n-k+2) k !(2 n-k) !} H\left(x-\frac{k-n}{2 n+2}\right), \\
i= & n, \\
j= & n+1 .
\end{aligned}
$$

The case of even $d$ is similar. Let $d=2 n+2, n \geq 0$. Set $A_{1}^{f}=\{-1 / 2\}$ and $B_{1}=\{1 / 2\}$. Set

$$
x_{k}=-\frac{1}{2}+\frac{k+1}{2 n+1}, \quad k=0, \ldots, 2 n-1
$$

and set

$$
\begin{aligned}
& A_{0}=\left\{x_{k} \mid k \text { is odd }\right\} \\
& B_{0}=\left\{x_{k} \mid k \text { is even }\right\}
\end{aligned}
$$

Again, the sequence $\mathcal{A}$ defined according to the above values of $A_{1}^{f}, B_{1}, A_{0}, B_{0}$, and with the sets (36) being empty, satisfies $\operatorname{Cond}(\gamma, q, \mathcal{A})$. Substitution into the formulas of Definition 4.10 yields the formula

$$
\begin{aligned}
& \alpha_{2 n+2}(x) \propto \frac{2^{4 n-2}(2 n+2) !(3 n) !}{(6 n+1) !(n+1) !}\left\{\frac{1}{3} H(x+1)+H(x-1)\right\} \\
& +\sum_{k=0}^{2 n-1} \frac{(8(k+1) / 3)^{\frac{1+(-1)^{k}}{2}}\left(2(2 n+1)^{2} /(2 n-k)\right)^{\frac{1+(-1)^{k+1}}{2}}}{(2 n+2 k+3)(6 n-2 k+1) k !(2 n-k-1) !} H\left(x-\frac{2 k-(2 n-1)}{4 n+2}\right) \\
& i=n \\
& j=n+1 .
\end{aligned}
$$

A simple calculation shows that, for the above example,

$$
\begin{aligned}
q_{0}^{\alpha_{2}} q_{1}^{\alpha_{2}} d \alpha_{2} & =\frac{\sqrt{3}}{4} H(x+1)-\frac{\sqrt{3}}{4} H(x-1) \\
& =\frac{\sqrt{3}}{2} q_{0}^{\beta} q_{1}^{\beta} d \beta \\
& \propto q_{0}^{\beta} q_{1}^{\beta} d \beta .
\end{aligned}
$$

See (35), above. This shows that, unlike the case $\Delta_{\gamma}=0$, it is necessary in the general case $\Delta_{\gamma}>0$, at least from the point of view of the physical inverse problem, to consider proportionality and not just equality.

Using the sequence $\left\{\left(\alpha_{d},\lfloor d / 2\rfloor-1,\lfloor d / 2\rfloor\right)\right\}_{d \geq 2}$ constructed above, one can compute the corresponding sequence of Jacobi matrices with associated indices,

$$
\left\{\left(J_{d},\lfloor d / 2\rfloor,\lfloor d / 2\rfloor+1\right)\right\}_{d \geq 2}
$$


where $J_{d}=J_{\alpha_{d}}$. The first few such matrices are:

$$
\left(\begin{array}{cc}
\frac{1}{2} & -\frac{\sqrt{3}}{2} \\
-\frac{\sqrt{3}}{2} & -\frac{1}{2}
\end{array}\right), \quad\left(\begin{array}{ccc}
0 & -\frac{\sqrt{3}}{2} & 0 \\
-\frac{\sqrt{3}}{2} & 0 & -\frac{1}{2} \\
0 & -\frac{1}{2} & 0
\end{array}\right), \quad\left(\begin{array}{cccc}
\frac{1}{6} & -\frac{1}{3} & 0 & 0 \\
-\frac{1}{3} & \frac{1}{6} & -\frac{\sqrt{3}}{2} & 0 \\
0 & -\frac{\sqrt{3}}{2} & -\frac{1}{6} & -\frac{1}{3} \\
0 & 0 & -\frac{1}{3} & -\frac{1}{6}
\end{array}\right) .
$$

Surprisingly, the matrices have a much simpler form than the p.d.f.s from which they are generated. We give an explicit formula for $J_{d}$ using the notation

$$
J_{d}=\left(\begin{array}{ccccc}
a_{1} & -b_{1} & 0 & \ldots & 0 \\
-b_{1} & a_{2} & -b_{2} & \ddots & \vdots \\
0 & -b_{2} & a_{3} & \ddots & 0 \\
\vdots & \ddots & \ddots & \ddots & -b_{d-1} \\
0 & \ldots & 0 & -b_{d-1} & a_{d}
\end{array}\right)
$$

Let

$$
\begin{aligned}
\kappa(d, k) & =\frac{\sqrt{k(d-1-k)}}{2(d-1)}, \\
\widetilde{\kappa}(d, k) & =\frac{\sqrt{k(d-k)}}{2(d-1)} .
\end{aligned}
$$

There are two cases, depending on whether $d$ is odd or even. We give the formula for odd $d$ first.

Let $d=2 n+3, n \geq 0$. Then each $a_{k}=0$, and

$$
\begin{aligned}
b_{k} & = \begin{cases}\kappa(d, k), & 1 \leq k \leq n-1, \\
\widetilde{\kappa}(d, k), & n+3 \leq k \leq d-1,\end{cases} \\
b_{n} & =\sqrt{2} \kappa(d, n), \\
b_{n+1} & =\sqrt{3} / 2, \\
b_{n+2} & =\sqrt{2} \widetilde{\kappa}(d, n+2) .
\end{aligned}
$$

In the case of even $d$, the diagonal entries of $J_{d}$ are no longer 0 . Let $d=2 n+2$, $n \geq 0$. Then

$$
\begin{aligned}
a_{k} & = \begin{cases}\frac{1}{d-1}, & 1 \leq k \leq n+1, \\
\frac{-1}{d-1}, & n+2 \leq k \leq d,\end{cases} \\
b_{k} & = \begin{cases}\kappa(d, k), & 1 \leq k \leq n-1, \\
\kappa(d, k-1), & n+3 \leq k \leq d-1,\end{cases} \\
b_{n} & =\sqrt{2} \kappa(d, n), \\
b_{n+1} & =\sqrt{3} / 2, \\
b_{n+2} & =\sqrt{2} \kappa(d, n+1) .
\end{aligned}
$$

By construction, the sequence $\left\{J_{d}\right\}_{d \geq 2}$ has the property that for every $d$, the $(\lfloor d / 2\rfloor,\lfloor d / 2\rfloor+1)$-entry of $\exp \left(t J_{d}\right)$ is proportional (and as it turns out, equal) to

$$
\frac{\sqrt{3}}{2} \sinh (-t) \text {. }
$$


From the point of view of constructing examples, it is useful to note that the map $\Phi$ respects order-preserving affine transformations of $\mathbf{R}$. To see this, let $\mathcal{T}$ denote the group of affine transformations

$$
x \stackrel{T_{a, b}}{\longmapsto} a x+b,
$$

where $a>0$ and $b \in \mathbf{R}$. Note that

$$
T_{a, b}^{-1}=T_{a^{-1},-b a^{-1}}
$$

and

$$
H\left(T_{a, b}(x)-\lambda\right)=H\left(x-T_{a, b}^{-1}(\lambda)\right) .
$$

Proposition 6.3. Let $(\alpha, i, j) \in \mathcal{D}, T_{a, b} \in \mathcal{T}$, and set $[d \gamma]=\Phi(\alpha, i, j)$. Then

$$
\Phi\left(\alpha \circ T_{a, b}, i, j\right)=\left[d\left(\gamma \circ T_{a, b}\right)\right] .
$$

Proof. Write

$$
\alpha(x)=\sum_{n=1}^{d_{\alpha}} w_{n} H\left(x-\lambda_{n}\right),
$$

and let $J_{\alpha}$ be orthogonally diagonalized as

$$
J_{\alpha}=O \Lambda O^{t}
$$

where $\Lambda=\operatorname{diag}\left(\lambda_{1}, \ldots, \lambda_{d_{\alpha}}\right)$. It follows from equation (8), Section 1.1, that $w_{n}=$ $(O(1, n))^{2}$ (setting $i=j=1$ ) and also that

$$
q_{i-1}^{\alpha}\left(\lambda_{n}\right)=\frac{O(i, n)}{O(1, n)}
$$

Define

$$
J=a^{-1} J_{\alpha}-b a^{-1} I
$$

and observe that $J$ has orthogonal diagonalization

$$
J=O\left(a^{-1} \Lambda-b a^{-1} I\right) O^{t} .
$$

Thus $J$ has eigenvalues $T_{a, b}^{-1}\left(\lambda_{1}\right), \ldots, T_{a, b}^{-1}\left(\lambda_{d_{\alpha}}\right)$, and therefore by (15), $J=J_{\beta}$, where

$$
\begin{aligned}
\beta(x) & =\sum_{n=1}^{d_{\alpha}} w_{n} H\left(x-T_{a, b}^{-1}\left(\lambda_{n}\right)\right) \\
& =\sum_{n=1}^{d_{\alpha}} w_{n} H\left(T_{a, b}(x)-\lambda_{n}\right) \\
& =\alpha \circ T_{a, b}(x) .
\end{aligned}
$$

Moreover, applying (39) to $J_{\beta}$, we obtain

$$
q_{i-1}^{\beta}\left(T_{a, b}^{-1}\left(\lambda_{n}\right)\right)=\frac{O(i, n)}{O(1, n)}=q_{i-1}^{\alpha}\left(\lambda_{n}\right) .
$$

Since $[d \gamma]=\Phi(\alpha, i, j)$, we may assume, without loss of generality, that

$$
\gamma(x)=\sum_{n=1}^{d_{\alpha}} q_{i}^{\alpha}\left(\lambda_{n}\right) q_{j}^{\alpha}\left(\lambda_{n}\right) w_{n} H\left(x-\lambda_{n}\right) .
$$


So,

$$
\begin{aligned}
\gamma \circ T_{a, b}(x) & =\sum_{n=1}^{d_{\alpha}} q_{i}^{\alpha}\left(\lambda_{n}\right) q_{j}^{\alpha}\left(\lambda_{n}\right) w_{n} H\left(T_{a, b}(x)-\lambda_{n}\right) \\
& =\sum_{n=1}^{d_{\alpha}} q_{i}^{\beta}\left(T_{a, b}^{-1}\left(\lambda_{n}\right)\right) q_{j}^{\beta}\left(T_{a, b}^{-1}\left(\lambda_{n}\right)\right) w_{n} H\left(x-T_{a, b}^{-1}\left(\lambda_{n}\right)\right) .
\end{aligned}
$$

This last expression shows that

$$
\begin{aligned}
{\left[\gamma \circ T_{a, b}(x)\right] } & =\Phi(\beta, i, j) \\
& =\Phi\left(\alpha \circ T_{a, b}, i, j\right),
\end{aligned}
$$

completing the proof.

Consider the sequence $\left\{\alpha_{d}\right\}_{d \geq 2}$ for which we derived an explicit formula. By Proposition 6.3, entries of the shifted sequence

$$
\left\{\alpha_{d} \circ T_{1,-2}\right\}_{d \geq 2}
$$

belong to $\Phi^{-1}\left(\left[d \gamma^{\prime}\right]\right)$, where

$$
\gamma^{\prime}(x)=\frac{1}{2} H(x-1)-\frac{1}{2} H(x-3) .
$$

The entries of (40) all have jump points in the closed interval [1,3], and so generate positive definite Jacobi matrices, which in turn can be used to generate the kind of physical model discussed in Section 1.3.

6.3. Remarks. We make a few remarks concerning the scope of the present results. It may be preferable in a particular context to treat, for example, the indices $i, j$, associated with a solution $(\alpha, i, j)$ as known, which greatly reduces the set of permissible solutions. But control over the resulting indices $i, j$ is implicit in the construction given in Definitions 4.6] and 4.10. In other words, it is a straightforward matter to modify the construction so as to generate specific indices, and generally speaking this greatly simplifies the procedure. For instance, in the case where $i=0$ and $j=\Delta_{\gamma}=d_{\gamma}-1$ are known, the solution set works out to be a $\Delta_{\gamma}$-dimensional manifold, diffeomorphic to an open ball. Similarly, if the number of jump points of a solution is known, the construction can be modified accordingly.

We have used the sign convention that next-to-diagonal elements of a Jacobi matrix are negative, which was consistent with our formulation of a particular physical example. But the opposite sign convention, whereby next-to-diagonal elements are positive, appears in the literature at least as often. Our results can be seen to hold equally well for these matrices, as follows. Let $J$ be a $d \times d$ Jacobi matrix (in our sense) and set

$$
E=\operatorname{diag}\left(-1,1,-1, \ldots,(-1)^{d}\right) .
$$

The matrix $H=E J E$ has positive next-to-diagonal elements. Note further that

$$
\begin{aligned}
\left\langle e_{j},(z I-H)^{-1} e_{i}\right\rangle & =\left\langle E e_{j},(z I-J)^{-1} E e_{i}\right\rangle \\
& =(-1)^{|j-i|}\left\langle e_{j},(z I-J)^{-1} e_{i}\right\rangle .
\end{aligned}
$$

Thus, the problem of determining which matrices $H$ have the property that the Green's function

$$
\left\langle e_{j},(z I-H)^{-1} e_{i}\right\rangle
$$


is proportional to an arbitrary prescribed function $f(z)$, is equivalent to the problem considered in this paper, with prescribed function $(-1)^{|j-i|} f$.

On another front, the results we have obtained for the case $\Delta_{\gamma}>0$ may have some interesting implications for continuous 1-dimensional systems. Suppose $[d \gamma] \in$ $\operatorname{Ran} \Phi, \Delta_{\gamma}>0$, and the jump points of $\gamma$ lie in $[a, b]$. Consider the set

$$
S=\left\{\alpha \mid(\alpha, i, j) \in \Phi^{-1}([d \gamma]) \text { for some } i, j\right\} .
$$

The jump points of every $\alpha \in S$ also lie in $[a, b]$. It seems that $S$ is not closed in, say, $L^{2}[a, b]$, and that the closure of $S$ ought to contain continuous p.d.f.s. If so, do the continuous p.d.f.s in the closure of $S$ correspond to physical systems, such as elastic continua, with exotic inverse spectral properties?

\section{ACKNOWLEDGEMENT}

Many thanks to Peter Hagedorn for his kind hospitality at the Dept. of Applied Mechanics, TU Darmstadt, where the original draft of this article was written.

\section{REFERENCES}

[Arv93] William Arveson, Improper filtrations for $C^{*}$-algebras: Spectra of unilateral tridiagonal operators, Acta Sci. Math. (Szeged) 57 (1993), no. 1-4, 11-24. MR 94i:46071

[BG87] Daniel Boley and Gene H. Golub, A survey of matrix inverse eigenvalue problems, Inverse Problems 3 (1987), 595-622. MR 89m:65036

[BR90] Riccardo Benedetti and Jean-Jacques Risler, Real algebraic and semi-algebraic sets, Actualités Mathématiques, Hermann, Paris, 1990. MR 91j:14045

[Brø83] Arne Brøndsted, An introduction to convex polytopes, Graduate Texts in Mathematics, vol. 90, Springer-Verlag, New York-Berlin, 1983. MR 84d:52009

[dBG78] Carl de Boor and Gene H. Golub, The numerically stable reconstruction of a Jacobi matrix from spectral data, Linear Algebra and its Applications 21 (1978), 245-260. MR 80i: 15007

[Gib] Peter C. Gibson, Spectral distributions and isospectral sets of tridiagonal matrices, Preprint.

[Gib00] Peter C. Gibson, Moment problems for Jacobi matrices and inverse problems for systems of many coupled oscillators, Ph.D. thesis, University of Calgary, 2000.

[Gla99] Graham M. L. Gladwell, Inverse finite element vibration problems, Journal of Sound and Vibration 211 (1999), 309-324.

[GS97] Fritz Gesztesy and Barry Simon, $M$-functions and inverse spectral analysis for finite and semi-infinite Jacobi matrices, Journal d'Analyse Mathématique 73 (1997), 267-297. MR 99c: 47039

[Lam97] Michael P. Lamoureux, Reflections on the almost Mathieu operator, Integral Equations and Operator Theory 28 (1997), 45-59. MR 98d:47068

[Sim98] Barry Simon, The classical moment problem as a self-adjoint finite difference operator, Advances in Mathematics 137 (1998), 82-203. MR 2001e:47020

[Tes00] Gerald Teschl, Jacobi operators and completely integrable nonlinear lattices, Mathematical Surveys and Monographs, vol. 72, American Mathematical Society, Providence, RI, 2000. MR 2001b:39019

[Var62] Richard S. Varga, Matrix iterative analysis, Prentice-Hall, New Jersey, 1962. MR 28:1725

Department of Mathematics, University of Washington, Seattle, Washington 98195

E-mail address: gibson@math.washington.edu 